Article

\title{
Responsibility and Performance Relationship in the Banking Industry
}

\author{
Halit Gonenc ${ }^{1}$ and Bert Scholtens $1,2, *$ (D) \\ 1 Department of Economics, Econometrics and Finance, University of Groningen, P.O. Box 800, \\ 9700 AV Groningen, The Netherlands; h.gonenc@rug.nl \\ 2 School of Management, University of Saint Andrews, The Gateway, North Haugh, St Andrews, Fife, \\ Scotland KY16 9SS, UK \\ * Correspondence: 1.j.r.scholtens@rug.nl; Tel.: +31-50-3637064
}

Received: 3 May 2019; Accepted: 13 June 2019; Published: 16 June 2019

\begin{abstract}
We study the relationship between financial performance and responsibility in the banking industry. Given the wide diversity in business models and operations, this relationship needs to be studied at the level of specific industries. We contribute to the debate about financial and social performance in the banking industry by using highly detailed responsibility and financial performance information, which helps to understand why this relationship exists and how the relationship evolves over time. We rely on a diverse international sample for the period 2002-2015 and use a wide range of financial performance measures next to various specific indicators for corporate governance, environmental, and social performance. By using simultaneous equation system estimations to address the causality between financial performance and responsibility, we find that the Tier-1 capital adequacy ratio is significantly and positively associated with responsibility indicators. As such, stronger institutions appear to be able to act in a more responsible manner and such responsibility signals banks' health. We also establish that the global financial crisis did have a profound impact on the finance-responsibility nexus. We show that there are changes in the underlying relationships in this nexus during the post-crisis period compared to the pre-crisis period. Furthermore, such changes are different between countries with high and low income, civil and common law, single and multiple supervision authorities, and central bank and non-central bank supervision.
\end{abstract}

Keywords: responsibility; financial performance; banking; financial crisis; country institutions; bank supervision

\section{Introduction}

This study investigates if and how responsibility in banking interacts with financial performance. Banks play a crucial role in the financial system and interact with economic development [1]. Responsibility relates to the performance of banks regarding governance, environmental, and social issues and operations that go beyond what is required by laws and regulations [2]. Recently, politically motivated groups and non-governmental organizations insist financial institutions take responsibility for social ills such as human rights violations and climate change by virtue of the activities they finance. For example, Amnesty International [3] requires banks commit to stop all financial activities related to illegal arms or arms destined to an illegal use. Thus, we try to clarify the relationship between how banks try to live up to such responsibilities and their financial performance, which we call the finance-responsibility nexus. We contribute to the academic debate by thoroughly investigating this nexus at the banking industry level for a large international sample. This allows us to account for banking specifics and for a more direct interpretation of the findings. It also allows us to use bank-specific measures of financial performance, such as capital ratios and net interest margins, 
and relate these to banks' performance on governance, social and environmental characteristics. This results in a richness of insights as to how responsibility and financial performance relate; such richness usually gets lost when multiple industries are investigated at the same time. Banks process financial information. As they specialize in the production and processing of information, we feel they are very well equipped and positioned to gather, assess and use non-financial information as well. As financial intermediaries manage risks and funds on behalf of other households, it is crucial they have a complete and detailed overview of what firms do and what the effects are. Both their financial structure and their business model set them apart from other types of business, which motivates our industry-specific analysis of the banking industry (next to the fact that several studies advocate the case for industry-specific analysis of the social and financial performance relationship, see [2]).

We are not the first to investigate the relationship between responsibility and financial performance for banks. Several other studies have tried to associate different characteristics of financial institutions to their responsibility, e.g., Anginer et al. [4] study the relationship between banks' capitalization strategies and executive compensation, and $\mathrm{Hu}$ and Scholtens [5] study how banks comply with international codes of conduct on social and environmental issues. Wu and Shen [6] argue that the more banks engage with responsibility, the better their financial performance. Jo et al. [7] establish that banks' environmental performance improves their operational efficiency and as such results in better financial performance. In their review study, Chih et al. [8] find that most research tends to confirm that banks' responsibility is to be significantly and positively associated with their size and returns. The reasoning behind these findings often is that stakeholders highly appreciate the efforts of banks regarding responsibility. Then, we wonder why most banks do not always act in a responsible manner. If being responsible were beneficial for financial performance, one would expect owners pressure bank managers to improve performance by acting in a responsible manner. However, this is not the case, as is being evidenced by ongoing criticism regarding the lack of responsibility in the banking industry [9]. Therefore, we reflect upon the reasons offered regarding the relationship between finance and responsibility in the banking industry and put these to the test.

The urge for responsibility results from the fact that private costs and benefits can differ from the social costs and benefits of doing business. This was clearly the case with the financial sector in the global financial crisis, where perverse incentive mechanisms resulted in bankruptcies and economic crises and led to the bailout of numerous banks [10-13]. The global financial crisis also resulted in calls for responsible conduct of the finance industry and for improvements in the quality of governance as well as legislation that explicitly addressed the responsibility of banks in their business operations $[14,15]$. Several initiatives have been set up to stimulate this debate and to try to achieve financial firms integrating responsibility in their business model. For example, the Equator Principles address how banks can account for social and environmental issues in project finance, and the Principles for Responsible Investment stimulate investors to value responsible investment to enhance governance, and in turn returns and improve risk management. Such initiatives highlight the importance of responsibility and the increase in attention for it after the global financial crisis [16,17]. Therefore, we also want to find out if the global financial crisis left its mark on the finance-responsibility nexus. More recent are the European Union's Circular Economy Action Plan and the Commission's Action Plan on Financial Sustainable growth. Given banks' crucial role in fostering economic growth, a thorough understanding about how responsible conduct affects their performance, and the other way around, seems crucial for achieving policy objectives.

We also investigate how income and country-level institutions affect this nexus [18]. Dixit [19] argues that a responsible business community can mitigate the impact of poor institutions and reduce corruption. Kotchen and Negi [20] study the Global Environment Facility and find that greater co-financing can be associated with better project evaluations. However, Cull et al. [21] report that the social and economic impacts of microfinance are only modest and that it involves substantial subsidies. Huang [22] establishes that the interaction between global financial markets and the economy is a key factor influencing sustainable development. Especially, output volatility has a negative effect on 
savings and increases natural resource depletion. In this respect, our study relates to the work of Liang and Renneboog [23] who investigate drivers of responsibility in general, and to that of Lins et al. [24] who study the role of responsibility with non-financial firms during the financial crisis. The paper also relates to Goss and Roberts [25] and $\mathrm{Wu}$ and Shen [6-26], who investigate how responsibility influences bank performance based on generic measures of responsibility.

We rely on an international sample for the period 2002-2015. Given the economic nature of banks, we use bank specific performance indicators, such as banks' net interest margins, cost-to-income ratio, non-performing loans, capital adequacy ratios, as the banking business is qualitatively different from other types of business [27-29]. For our proxies of responsibility, next to the generic indicators that are used in the empirical literature so far $[6,7,26,30]$, we also use several components of social, environmental and governance performance and relate these to the bank specific indicators. For example, in the social realm, we account for product responsibility, health and safety, human rights, community, employment, diversity, training and development. As to environment, we account for resource use reduction, product innovation, and emission reduction. For governance, we investigate board structure and functions, compensation policy, shareholder rights, vision and strategy. This granularity is important because aggregated responsibility indicators are not very informative [31].

Further, we study whether the global financial crisis of 2008-2009 did affect the finance-responsibility nexus, as it gave rise to a lot of pressure from supervisors and regulators regarding improving banks' health and the way in which they operate [24,32,33]. Responsibility might be a strategy to help protect banks from such pressure. Banks are important agents affecting economic development and therefore are regulated and supervised carefully. However, the recent global banking crisis and its aftermath severely damaged banks' reputations. This is demonstrated by bank bailouts and scandals like interest rate rigging. In order to restore their role and reputations, bank regulation and supervision was intensified [13]. Further, previous studies suggest that differences in economic and institutional development across countries might influence the effectiveness of and interest in responsibility [24,34]. Therefore, as to their impact on the finance-responsibility nexus, we also investigate the role of per capita income, the legal system, and the design of bank supervision $[24,35]$ in the relationship between social and financial performance in the banking industry. We study if differences in those institutional developments across countries help explain how the recent global financial crisis shapes the relationship between social and financial performance.

The main findings are that we cannot support the view that responsibility systematically improves financial performance in the banking industry. However, we establish that capitalization (Tier-1 ratio) significantly and positively relates to responsibility indicators. We do not observe other financial or non-financial performance indicators having a consistent relationship with other proxies for social performance. We find that the global financial crisis left its mark on the interaction between financial and responsibility performance in the banking industry. In particular, it shows that the finance-responsibility nexus did weaken after the crisis. Further, economic development, legal systems, and the way in which bank supervision is organized do matter for the finance-responsibility nexus.

To find out about the finance-responsibility nexus in the banking industry, we first briefly refer to the studies that relate social and financial performance; next, we introduce the literature that focuses on this relationship within the banking industry and position our study before we present the hypotheses that will be tested in the remainder of this manuscript.

\section{Corporate Social Responsibility and Financial Performance}

The Commission of European Communities [36] defines Corporate Social Responsibility (CSR) as companies integrating social and environmental concerns in their business operations and voluntarily engaging with their stakeholder base. This definition accounts for a role of CSR within the firm. Dahlsrud [37] observes that CSR has distinct dimensions, namely economic, environmental, social, stakeholder, and voluntary. 
The research on CSR has generated a wide array of ideas as to why and how the non-financial performance of a firm might be associated with its financial performance. Margolis and Walsh [38] argue CSR has become a trend, as society holds firms responsible for the problems they cause and expects they contribute to their solution. Bénabou and Tirole $[39,40]$ argue that as information about business' practices has become accessible, stakeholders can more easily assess whether a firm acts in line with their interests. Further, they contend that the costs of externalities such as pollution and the awareness about these costs have risen significantly. Due to their increasingly globalized activities, companies face new problems to which they have to respond. Then, corporate responsibility is a way to manage firms' externalities [2].

The study of financial performance and CSR has yielded a very extensive empirical literature. Friede et al. [41] report that more than 2000 studies investigate this relationship. Most studies arrive at a small but significant and positive association between the two (see also Margolis et al. [42]). Industry factors appear to play a significant role, but there is little theory in this respect. Waddock and Graves [43] observe that there are substantial differences in CSR disclosure across industries. Therefore, Cottrill [44] and Heal [2] contend that investigations failing to incorporate industry level realities may be fatally deficient. Boutin-Dufresne and Savaria [45] and Heal [2] argue that firms in a particular industry can be either more or less socially responsible because of the specific nature of their activities. Simpson and Kohers [46] argue that differences between industries with regard to CSR are so dominant that research needs to stick to the single industry perspective. Fernando et al. [47] contend that corporate environmental policies that mitigate environmental risk exposure create shareholder value (see also [48]).

The majority of studies about financial performance and CSR base their hypotheses on taxonomy provided by Preston and O'Bannon [49]. Rivera et al. [50] try to substantiate the different ideas based on economic theory. Preston and O'Bannon [49] first question the sign of the relationship: It may be positive, negative or non-existent. Next, they question causality: Does responsibility affect financial performance, or is it the other way around? From a mix of different notions-sometimes labelled as theories in the literature, they arrive at six testable hypotheses. First is that there is a positive relationship between social and financial performance and that social performance is leading. An example is the social impact hypothesis, which holds that high levels of responsibility lead to high levels of financial performance. This results from stakeholder theory as proposed by Freeman [51] (see also [50]). This theory assumes that a firm needs to invest in its relationships with key stakeholders to be financially successful [52,53]. Then, investments in CSR contribute to better relations with a firm's stakeholders and ultimately lead to increased financial performance [43]. Lins et al. [24] argue that CSR activities especially help build social capital and trust (see also $[19,20]$ ).

Second is that there is a negative relationship and that social performance is leading. An example is the trade-off hypothesis, which closely relates to the view of Friedman [54] who argued that there are few measurable benefits of CSR while the costs are substantial (see also [50]). For example, managers would invest in responsibility to pursue their own interests, which would not maximize shareholder wealth. As such, it could worsen the competitive position of the firm and reduce its financial performance. Harris and Raviv [55] argue that shareholders should have more control over important decisions, but assume they all have the same preferences. However, Dimson et al. [53] argue that some investors in fact are activists and show that activist owners who focus on corporate social responsibility can improve companies' returns and governance.

Third is that there is a positive relationship with financial performance leading social performance. An example is the available funds view, which holds that investments in CSR are costly, and therefore depend on the financial resources of the firm [56] (see also [50]). Managers can invest internally available funds in environmentally or socially advantageous activities without being restricted with the difficulties of raising external financing. Even though managerial control on internal cash flows raises concerns for the classical agency problems [57,58], spending those funds on responsible investments may reduce management's power and, in turn, create benefits for all stakeholders [59]. 
Fourth is that financial performance is leading and there is a negative relationship with social performance. Here, the managerial opportunism view is an example as it argues because managers act to maximize their own private benefits at the expense of shareholders' and other stakeholders' interests [60,61] (see also [50]). For example, after good financial performance managers like to reward themselves and spend less on CSR. However, after poor financial performance management may spend more on CSR or philanthropy to cover up or justify its poor financial performance [22,49]. Preston and O'Bannon [49] also allow for a positive or negative bidirectional relationship (see also [43]), i.e., their fifth and sixth hypothesis respectively, but do not offer a specific reasoning as to why this might be the case.

\subsection{Responsibility in the Banking Industry}

Most empirical studies control for industry effects in the regression models and include size, and sometimes R\&D and marketing, in the regression model. It shows that industry effects matter [2,44]. This motivates the case for studying the finance-responsibility nexus at the industry level. Many studies exclude financial firms from their analysis because they are special. In particular, banks' leverage is out of range with that of non-financial firms and they are subject to much more intense regulation than other firms [62]. This is because they create and manage money and, from an economic perspective, are intermediaries: Their services help clients manage intertemporal financial surpluses and deficits as well as financial risk $[63,64]$. To do so, banks take on risks themselves for which they are rewarded by fees and markups. Therefore, banks' balance sheets and income statements are very different from that of other industries as they predominantly consist of (intangible) financial assets [62].

The unique characteristics of banks motivated scholars to suggest that the banking industry might also be different when it comes to the responsibility-performance nexus $[2,43,45,46]$. Several country studies investigate CSR in the financial industry (e.g., [15,18,46,65] for the US; [66] for the Netherlands; [67] for Lebanon; [68] for Spain; [69] for Bangladesh; [70] for India; [71] for Egypt; [72] for Nigeria; [73] for Pakistan; [74] for the Czech Republic. The ways in which responsibility is dealt with shows wide variation: from actual resource usage to generic ratings, and from topical aspects to broad categories. The predominant finding is that responsibility results in financial outperformance ([66,74] being an exception). Cornett et al. [15] examine banks' responsibility in the US in the context of the global financial crisis and establish that socially responsible banks outperform. In addition, there are several studies using an international sample (for example [4-8,26,75-78]. The results from multi-country studies generally point in the same direction as those for individual countries. This line of research suggests it is worthwhile to account for economic development and institutional design in our research framework (see also $[13,23,24]$ ).

Chih et al. [8] provide an overview of the literature and conclude that many key characteristics of social performance are positively associated with financial ratios and performance indicators in the banking industry (see also [79]). Wu and Shen [6] argue that the more banks engage with responsibility, the better their financial performance as reflected in several bank efficiency and performance ratios. They confirm these findings in later research [26,78]. Ciciretti et al. [76] confirm this too and suggest more responsible banks have lower cost of debt and equity. Mallin et al. [77] study the case of Islamic banks and arrive at similar conclusions. They suggest causality runs from financial performance to responsibility. Platanova et al. [80] also study Islamic banking but conclude responsibility precedes financial performance. Jo et al. [7] argue banks' environmental performance improves their operational efficiency and as such results in better financial performance. Most of these studies suffer from small sample size; only $[4,7,24,65,76]$ report more than 1000 bank-year observations in their sample. The use of responsibility and financial performance variables differs widely and we find that hypotheses are not always clearly stated or tested. Related is variety in the use of estimation methods, especially when it comes to addressing endogeneity problems. This criticism is reminiscent of the critical reflection regarding the study of CSR in general $[42,81,82]$. 
Hence, we feel it is important to conduct an industry-specific study for the banking industry regarding the finance-performance nexus. We try to add value to the literature by using a broad set of both financial performance and social performance variables, where the existing literature usually focuses on just a few generic measures that allow cross-industry comparison. Given the two highly different types of variables, we will engage in an approach that controls for the direction of the causation by using a three Stage Least Square (3SLS) estimation. As such, we take advantage of the correlation in the error terms to arrive at estimates that are more efficient and perform a generalized least squares estimation for this 3SLS process that creates a consistent estimate for the covariance matrix of the equation disturbances in 3SLS estimations. This allows us to pinpoint the interactions between the different types of performance in detail. Further, we rely on a large international sample $[7,24]$, specifically compare the nexus before and after the global financial crisis [15,33], and account for development and institutions [34,43]. In our view, such a contribution is of interest as banks are under scrutiny of their regulators and the public at large, especially since the global financial crisis. By using a broad sample for a prolonged period, and by investigating whether the global financial crisis made a difference, we want to establish what drives banks' responsibility, if there is a cost, and whether development and institutions matter.

\subsection{Hypotheses}

To investigate the finance-responsibility nexus for banks, we have four hypotheses. For the first two, we closely follow the setup suggested in Preston and O'Bannon (1997) [49] and further developed in Rivera et al. (2017) [50]. The background of these hypotheses relates to the response to the concerns about banks' health and their way of doing business as, traditionally, banks are regarded as the culprits in all types of societal ills and wrongdoing [83]. Consequently, especially banks will invest in their responsibility policies as a response to such pressure $[2,46]$. Their governance may be affected because of extensive regulation $[84,85]$, and wide diversity of their stakeholders, which can result in potential conflicts [86]. Many operations of banks are subject to strict regulation by monetary and supervisory authorities and so is the composition of the board and the requirements regarding the skills and character of its members [87]. The extensive regulation gives individual banks very little leeway to deviate from the industry standard as set by their regulators.

We first assume there is a causal relation running from responsibility to financial performance (H1). Rivera et al. mention there are several views as to why this might be the case. For example, they suggest it is neoclassical finance theory, which sees responsibility as an attribute that is sold. Further, it signals the firm's mission or balances claims of multiple stakeholders. They also relate it to institutional theory and to the natural resource view of the firm. Last is that Rivera et al. [50] assume the relationship can be negative too when environmental protection consumes financial resources. When the relationship is significant and positive, this would confirm these different views. Motivated by Waddock and Graves [43], Rivera et al. [50] also are open to financial performance driving social performance. Here they assume good management and social performance are synonyms, or that financial resources enable social performance. Therefore, we also test whether causality runs from financial performance to responsibility $(\mathrm{H} 2)$. When this relationship is statistically significant and positive, we assume it supports the available funds view. When negative, it suggests the opportunism view holds (firms improve financial performance at the expense of social and environmental wellbeing).

Our third hypothesis relates to the impact of the global financial crisis on the relationship between responsibility and financial performance. Here, we specifically relate to $[15,33]$ for the US, who show that responsibility conditions firms' financial performance in the crisis period. As we have to make do with a lack of theory, our third hypothesis is of an exploratory nature. We test whether the crisis did significantly change the ways in which the two performances interact.

Our fourth and last hypothesis is also of a probing nature as we lack a grounding framework. This hypothesis relates to the role of economic and institutional development in relation to the finance-responsibility nexus. As there are few studies to highlight the potential role of these $[24,34,43]$, 
but there is no clear-cut transmission mechanism as to how and why so, we feel we can only have this as an exploratory hypothesis. It holds that economic and institutional development matter regarding the finance-responsibility nexus. The null being there is no difference between any country subsets. We will relate the role of economic and institutional development on the finance-responsibility to the banking industry's response to the global financial crisis as well.

\section{Materials and Methods}

\subsection{Materials}

We investigate the relationship between banks' responsibility and financial performance. We use an international sample of firms for the period 2002-2015. We extract financial and non-financial firms having data for responsibility scores in the ASSET4 database of Thomson Reuters Worldscope/Datastream. We exclude countries with less than 10 observations during the sample period. To be included in our final sample, we require all firms having firm level financial items from Worldscope/Datastream. We use ASSET4 data to measure responsibility for several reasons. First is that it is a global dataset and covers extensive data items with more than 7000 firms and 40,000 firm/year observations for the time period of 2002-2015. Further, it includes details of more than 250 responsibility items as well as encompassing scores for environmental performance, social performance, and governance. Several academic studies use this database too when examining the interaction between CSR and financial performance [30,79,88-91].

ASSET4 consists of four generic pillars, which represent different dimensions: corporate governance, economic, environmental, and social. We leave out the economic pillar, as we specifically want to associate financial performance with responsibility measures. ASSET4 is based on (normalized) z-scores, which reveals a company's performance relative to the average performance of all other rated companies. The use of such ratings information has its limitations [90,92]. Firstly, the ratings derive from the firm's definition and evaluation of its non-financial performance and are not directly related to scientific measures of sustainable development. To this date, however, this concern applies to all responsibility ratings and seems insurmountable as long as there is no standardized and independent auditing and verification with respect to non-financial performance. Another limitation of responsibility ratings is that they are largely process-based as they primarily focus on managerial principles and processes. They mainly capture a firm's intention, and not its effort, to address corporate social, governance, and environmental issues. Examples of process-based measures include data points that answer questions such as "Does the company have a policy regarding the independence of the board?", or "Does the company monitor the impact of its products or services on consumers or the community more generally?", or with respect to financial firms "Does the company show in its role as an asset manager that it promotes socially responsible investments?". Orlitzky et al. [81] stress that researchers must decide whether process-based measures are as appropriate measures of non-financial performance. They argue that their use is equivalent to acknowledging effort. From an economic point of view, effort would pertain to resources used in the production process and it should somehow show up in the financial information, but the efficiency of these processes can highly differ. Another concern is the aggregation of data. This assumes the data is commensurable. We think it is unlikely that this assumption holds for responsibility indicators. In addition, aggregation requires a decision about the weight to apply to each component (see [93] for a discussion about commensurability and fungibility as well as the role of taste and values). Even though Asset4 aggregates information about detail items for governance environmental and social performance, there is no scientific framework that can be used to arrive at this decision.

We use the banks' scores on corporate governance performance, environmental performance, and social performance as proxies for corporate social responsibility and test our hypotheses for these measures. The information about governance, environmental and social performance is available at a general level as well as at a more detailed level. The definitions of responsibility variables are in 
Appendix A, along with a definition of our proxies for bank specific financial performance variables such as the ratio of net interest income to earning assets, Tier 1 capital adequacy ratio, the ratio of non-performing loans to total loans, the cost to income ratio, and excess stock market returns. Banks report these variables to inform their stakeholders. The net interest margin reflects the business banks engage in; banks with more lending operations will have higher margins than those that engage in advising and mediating, where fees and provisions are the main revenues. Hence, this is not reflecting performance but merely revealing the source of the bank's revenues. Tier 1 is a capital adequacy ratio that reflects the solvency or capital strength of the banks. The Tier 1 ratio reflects the highest quality of capital held in relation to the risk-weighted assets; the total capital adequacy ratio, as an alternative proxy for the quality of capital, includes all capital components (results with this proxy are highly similar but not reported for the sake of brevity). A bank's non-performing loans signal the quality of the loan portfolio; a high ratio implies relatively poor quality. The cost to income ratio is an indicator of bank efficiency: banks with low ratios are most efficient. The excess stock market return signals the investor's valuation of the bank in relation to the general stock market. To account for the possibility of mistakes or outliers, all variables are winsorized at the bottom and top one percentile levels. The final sample is unbalanced pooled cross-sectional data with more than 2400 bank/year observations.

Table 1 reports the descriptive statistics of the overall sample and correlations coefficients. Our investigation is motivated by an industry-specific analysis suggesting that there is potential to augment the analysis of $[15,24,33]$. This table also compares the pre-crisis (2002-2007) and the post-crisis (2010-2015) years and shows that (1) environmental responsibility in the banking industry improved while governance and social performance deteriorated, and (2) all financial performance proxies improved in post crisis period (2010-2015) relative to the pre-crisis period (2002-2007), as the differences are statistically significantly different from zero. These characteristics suggest we should investigate our hypothesis about the changing relationship between financial and non-financial performance in relation to the crisis. We also report correlations coefficients between responsibility scores and financial performance proxies in the banking industry. This too shows a high correlation between environmental and social performance (0.77).

We now turn to the ways in which we will test the hypotheses about the direction and sign of the CSR and financial performance relationship in the banking industry, the impact of the global financial crisis on these relationships, as well as on the role of development and institutions.

\subsection{Methods}

To test the sign and direction for our hypotheses, we investigated the relationships between financial performance and responsibility and accounted for their sequence. Our aim was to identify if and which of the financial performance variables might have determined the responsibility scores, and vice versa. Most of the empirical literature on this relationship in the banking industry tended to rely on Ordinary Least Square (OLS) estimations. Weighted Least Square (WLS) estimation takes unequal representations of sample countries with the different number of observations into account for especially a few countries having larger number of observations. As we had a large international sample, WLS would provide an attractive alternative estimation method. However, WLS estimations do not consider the issue that the direction of causation between governance, environmental and social scores and firm financial performance is unknown. Therefore, we had to amend this strategy. To this extent, we controlled for the direction of the causation with a system of equations using a three Stage Least Square (3SLS) estimation, which took advantage of the correlation in the error terms to arrive at estimates that were more efficient, as widely documented in the literature (e.g., $[42,94,95])$. By using two equations, we designated both financial performance and responsibility as endogenous variables. The predictive values of both endogenous variables were determined using all exogenous variables in the two equations and the instrumental variables identified the difference between the equations. We performed a generalized least squares (GLS) type estimation for the 3SLS process which created a consistent estimate for the covariance matrix of the equation disturbances. 
The first equation regressed firm financial performance against the (separate) responsibility scores (i.e., governance, environment, and social). As controls, we used one-year lags of the financial performance variable, size, the ratio of loans to deposits (LoanDeposit). Bank size was important because larger banks can have higher financial and social performance than small banks do since they can create efficiency and draw public attention [15]. The ratio of loans to deposit indicates available access funds for banks to pursue responsibilities better [15]. We also controlled with the country level private credit percent of GDP (Pr.Crd.GDP) by deposit money banks and other financial institutions. This variable controlled for the size of the banking sector across countries, reflecting institutional development. This is in line with [23] who examined legal origin when explaining responsibility scores Private credit to GDP is a proxy that closely aligns with our methodology as it allows for variation over time [96]. We identified lagged financial performance and private credit to GDP as instruments of the financial performance equation. We expected that, as the size of the credit market was different across countries, private credit to GDP helped determine the financial performance, but not necessarily responsibility, which primarily was driven by country and industry institutions and regulations and thus depended on country and industry practices $[23,96]$. Further, we examined how the causal relationship between corporate social and financial performance in the banking industry differed during the crisis period relative to the pre- and post-crisis periods. We took years 2008 and 2009 as crisis years (based on [97-99]) and introduced a dummy to compare this period with others. Then, we interacted the dummy representing the crisis period with responsibility in the financial performance equations as well as with financial performance in the equations determining the responsibility scores [15-24]. Thus, the first equation was as follows:

$$
\begin{aligned}
& \text { FinancialPerformance }_{i t}=b_{0}+b_{1} \text { FinancialPer formance }_{i t-1}+b_{2} \text { ResponsibilityScore }_{i t}+b_{3} \text { Crisis }_{\text {S }} \\
& +b_{4} \text { Crisis } * \text { ResponsibilityScore }_{i t}+b_{5} \text { Size }_{i t}+b_{6} \text { LoanDeposit }_{i t}+b_{7} \text { Pr.Crd.GDP } P_{i t}+e_{i}
\end{aligned}
$$

Next, Equation (2) had governance, environment, and social responsibility scores as the dependent variables and one financial performance measure was included each time as an explanatory variable. Further, we used two additional instrumental variables, namely the averages of the scores by country/year and those by country/industry. We computed peer group averages as instruments for each responsibility score separately. However, [100] showed that the choice of an instrument as the mean of the group's dependent variable produced inconsistent estimates and suggested the fixed effects estimator was consistent and should be used instead. Unfortunately, responsibility scores do not have sufficient variation to allow for the use of bank fixed effects. [48] used the peer group averages as instruments for a focal firm's financial policies and motivated this because of the existing empirical evidence in the literature for the impact of peer firms' polices on individual firm-level financial policies. This is quite similar for the case of responsibility policies. Therefore, the analysis was performed with the help of the second equation:

$$
\begin{gathered}
\text { ResponsibilityScore }_{i t}=b_{0}+b_{1} \text { FinancialPerformance }_{i t}+b_{2} \text { Crisis }_{1}+b_{3} \text { Crisis } * \text { Financial } \\
\text { Performance }{ }_{i t}+b_{4} \text { Size }_{i t}+b_{5} \text { LoanDeposit }_{i t}+b_{6} \\
\text { Mean_RESSCORE }(\text { Country } / \text { Year })_{i t}+b_{7} \text { Mean_RESSCORE }(\text { Country } / \text { Industry })_{i t}+e_{i}
\end{gathered}
$$

To account for development and institutions (as motivated in $[4,18,19,24,34]$ ), we split the sample countries along per capita income, legal system, and along their way of organizing supervision of the banking industry and ran regressions based on two equations above. 
Table 1. Descriptive statistics for financial performance measures and responsibility scores by industry. This table reports the number of firm/year observations, the mean and median values of the financial performance measures and responsibility scores for banking sample and provides a comparison between post and pre crisis years and correlation coefficients of performance scores and responsibility scores for the sample. The definitions of variables are in Appendix A. The sample period is from 2002 to 2015. The significance of differences between means and medians is based on a t-test for mean differences and Wilcoxon rank-sum test for median differences, and ${ }^{* * *}$ denotes statistical significance at the $1 \%$ levels.

\begin{tabular}{|c|c|c|c|c|c|c|c|c|c|c|c|c|c|}
\hline & \multicolumn{3}{|c|}{ Total } & \multicolumn{3}{|c|}{ Pre Crisis } & \multicolumn{3}{|c|}{ Post Crisis } & \multicolumn{4}{|c|}{ Post Crisis Minus Pre Crisis } \\
\hline & $\mathbf{N}$ & Mean & Median & $\mathbf{N}$ & Mean & Median & $\mathbf{N}$ & Mean & Median & Mean & & Median & \\
\hline CGVSCORE & 2432 & 46.04 & 48.58 & 605 & 50.59 & 58.95 & 1436 & 44.68 & 46.17 & -5.91 & $* * *$ & -12.79 & $* * *$ \\
\hline ENVSCORE & 2432 & 48.85 & 42.91 & 605 & 47.54 & 35.89 & 1436 & 49.88 & 47.71 & 2.33 & & 11.82 & \\
\hline SOCSCORE & 2432 & 54.52 & 55.26 & 605 & 58.13 & 61.89 & 1436 & 52.94 & 51.83 & -5.19 & $* * *$ & -10.06 & $* * *$ \\
\hline Net Interest Margin & 2432 & 0.0270 & 0.0236 & 605 & 0.0235 & 0.0215 & 1436 & 0.0284 & 0.0254 & 0.005 & $* * *$ & 0.004 & $* * *$ \\
\hline Tier1 Cap_Adq Ratio & 1852 & 0.1177 & 0.1143 & 211 & 0.0923 & 0.0840 & 1292 & 0.1248 & 0.1210 & 0.03 & $* * *$ & 0.04 & $* * *$ \\
\hline Non Performing Loans & 2266 & 0.0293 & 0.0178 & 550 & 0.0150 & 0.0079 & 1346 & 0.0354 & 0.0211 & 0.02 & $* * *$ & 0.01 & $* * *$ \\
\hline Cost to Income Ratio & 1913 & 0.4203 & 0.4185 & 429 & 0.5441 & 0.5351 & 1202 & 0.3660 & 0.3761 & -0.18 & $* * *$ & -0.16 & $* * *$ \\
\hline Excess Stock Return & 2432 & 0.0240 & 0.0109 & 605 & 0.0130 & 0.0086 & 1436 & 0.0259 & 0.0125 & 0.013 & & 0.004 & \\
\hline Correlations & & \multicolumn{2}{|c|}{ [1] } & [2] & [3] & \multicolumn{2}{|c|}{ [4] } & [5] & [6] & \multicolumn{2}{|c|}{ [7] } & \multicolumn{2}{|c|}{ [8] } \\
\hline CGVSCORE & [1] & \multicolumn{2}{|c|}{1} & & & & & & & & & & \\
\hline ENVSCORE & [2] & \multirow{2}{*}{\multicolumn{2}{|c|}{$\begin{array}{l}0.4205^{*} \\
0.4923 \text { * }\end{array}$}} & 1 & & & & & & & & & \\
\hline SOCSCORE & [3] & & & $0.8190 *$ & 1 & & & & & & & & \\
\hline Net Interest Margin & [4] & \multicolumn{2}{|c|}{$\begin{array}{l}0.4923^{*} \\
-0.0095\end{array}$} & $-0.2060 *$ & -0.0980 * & \multicolumn{2}{|c|}{1} & & & & & & \\
\hline Tier1 Cap_Adq Ratio & [5] & \multicolumn{2}{|c|}{0.0332} & $-0.0842 *$ & -0.0536 & \multicolumn{2}{|c|}{$0.2256^{*}$} & 1 & & & & & \\
\hline Non Performing Loans & [6] & \multicolumn{2}{|c|}{$-0.1026^{*}$} & $0.1126^{*}$ & $0.0870 *$ & \multicolumn{2}{|c|}{0.0366} & 0.0237 & 1 & & & & \\
\hline Cost to Income Ratio & [7] & \multicolumn{2}{|c|}{0.0315} & 0.2469 * & 0.3079 * & \multirow{2}{*}{\multicolumn{2}{|c|}{$\begin{array}{c}-0.2655^{*} \\
0.0760 \text { * }\end{array}$}} & -0.2455 * & 0.0215 & \multicolumn{2}{|c|}{1} & \multirow{2}{*}{\multicolumn{2}{|c|}{1}} \\
\hline Excess Stock Return & [8] & \multicolumn{2}{|c|}{$-0.0664 *$} & -0.0282 & -0.028 & & & $0.1102 *$ & $-0.0822 *$ & \multicolumn{2}{|c|}{-0.0443} & & \\
\hline
\end{tabular}




\section{Results}

In this section, we provide the analysis of our regression estimations where we use bank specific financial performance measures and both encompassing and detailed responsibility indicators. We first provide a detailed analysis of various responsibility and financial performance measures and their interrelations. Then, we investigate whether the global financial crisis affected the finance-responsibility nexus in the banking industry. Last is the analysis of the influence of per capita income, legal system, and the way in which bank supervision is organized.

\subsection{Main Analysis}

Table 2 presents the results of 3SLS estimations of the simultaneous equation system regarding the effect of a bank's responsibility (corporate governance (CGVSCORE), environmental (ENVSCORE) and social (SOCSCORE) scores) on financial performance, as well of that of financial performance on these responsibility indicators (we also perform OLS and WLS estimations to provide a comparison across alternative estimations methods and report the findings in Appendix C). At this stage, we ignore the role of different types of development (i.e., economic, institutional, regulatory), but leave this for our analysis of the impact of the global financial crisis (Sections 4.2 and 4.3). The results are reported for CGVSCORE, ENVSCORE, and for SOCSCORE separately. For all three responsibility scores Table 2, we report the results for financial performance equations in the first part, and the results for responsibility equations in the second part.

Table 2 first relates CGVSCORE to banks' financial performance indicators. We find that CGVSCORE and Net Interest Margin (NIM) positively and significantly affect each other, which supports both $\mathrm{H} 1$ and $\mathrm{H} 2$. This suggests that especially banks with more lending business, i.e., more traditional commercial banks, will have better governance compared to those with relatively more fee-based income. The other significant effect we observe is a positive effect of banks' TIER1 capital adequacy ratio on CGVSCORE. In this case, the causality appears to run from finance to responsibility and not the other way. This confirms the available funds view (H2) regarding the relationship between governance and financial performance in the banking industry [50]. Next we report the results for the relationship between ENVSCORE and financial performance. We find that ENVSCORE significantly decreases NIM and increases Cost to Income Ratio (CIR). These results suggest that better environmental performance lowers banks' efficiency and that banks high on environmental responsibilities are not the most cost-efficient ones. This finding confirms the trade-off view $(\mathrm{H} 1)$ as more responsibility reduces banks' financial efficiency. Banks with higher non-performing loans and cost to income ratio have higher environmental performance. However, the positive and significant effect of Tier 1 capital adequacy on ENVSCORE indicates that banks adequately backed up by equity improve their environmental performance, which confirms the available funds hypothesis (H2). The results with SOCSCORE show that, as in the case of environmental performance, a higher SOCSCORE is associated with a higher cost to income ratio and vice versa, supporting H1. This indicates that being socially responsible comes at a cost. On the other hand, the positive and significant effects of NIM, Tier1 and Excess Return on SOCSCORE show that banks with sufficient margin, better capitalization and outperformance have higher social responsibility. This confirms the available funds hypothesis (H2).

Both OLS and WLS estimations reported in Appendix C provide similar findings as 3SLS estimations do. Even though there are some differences across alternative estimations, the similar estimation results of 3SLS with especially WLS in most of the cases indicate that our 3SLS estimations are not affected by countries represented with varying number of observations in our sample. These comparative results indicate the importance of the 3SLS estimations, where two equations are simultaneously estimated, to be able to identify the direction of the effect.

Among the control variables, the estimated coefficients of the lag of financial performance proxies are always highly significant and most of the time reduce the role of country level variable, Private Credit to GDP. In the responsibility equations, size and two instrumental variables (the means of responsibility scores by country/year and by country/industry) are major determinants of responsibility 
indicators. Moreover, according to Variance of Inflation Factors (VIFs) reported in Appendix D, we do not detect any major correlations among independent variables. The reason for VIFs being a little higher for the means of responsibility scores of CGVSCORE by country/year and by country/industry is that CGVSCOREs are stable over the sample period. However, the VIFs of the other two responsibility scores are very low.

Based on Table 2, we conclude that there mainly is support for the available funds hypothesis, especially in the case of the Tier- 1 capital adequacy ratio. There is some support for the trade-off hypothesis and none for the social impact and opportunism hypotheses. In the case of corporate governance and the net interest margin, there is some evidence of positive synergy.

Table 2. The relationship between responsibility scores and financial performance for the banking industry. This table reports 3SLS estimations for simultaneous equation system of the relationship between responsibility scores and bank specific financial performance measures for the effect of crisis years with CGVSCORE, ENVSCORE, and SOCSCORE. The definitions of variables are in Appendix A. All regressions control year fixed effects. The sample period is from 2002 to 2015 . Robust standard errors presented in brackets are clustered at the firm level, and ${ }^{* *}, * *$, and ${ }^{*}$ denote statistical significance at the $1 \%, 5 \%$, and $10 \%$ levels.

\begin{tabular}{|c|c|c|c|c|c|}
\hline \multicolumn{6}{|c|}{ Corporate Governance (CGVSCORE) } \\
\hline & $\begin{array}{l}\text { Net Interest } \\
\text { Margin }\end{array}$ & Tier1 Cap_Adq & $\begin{array}{c}\text { Non } \\
\text { Performing } \\
\text { Loans }\end{array}$ & $\begin{array}{c}\text { Cost Income } \\
\text { Ratio }\end{array}$ & Excess Return \\
\hline \multicolumn{6}{|c|}{ Financial Performance Equation } \\
\hline \multirow{2}{*}{$\begin{array}{c}\text { Financial } \\
\text { Performance_lag1 }\end{array}$} & $0.918^{* * *}$ & $0.830^{* * *}$ & $0.979^{* * *}$ & $0.598^{* * *}$ & $-0.064^{* * *}$ \\
\hline & [0.006] & {$[0.014]$} & {$[0.009]$} & [0.019] & {$[0.020]$} \\
\hline \multirow[t]{2}{*}{ CGVSCORE } & $0.098^{* *}$ & 0.353 & 0.219 & -0.008 & -0.04 \\
\hline & [0.047] & [0.245] & {$[0.160]$} & {$[0.023]$} & [0.026] \\
\hline \multirow[t]{2}{*}{ Size } & $-0.028^{* * *}$ & 0.004 & -0.01 & $0.014^{* * *}$ & $-0.010^{* *}$ \\
\hline & [0.009] & [0.045] & {$[0.029]$} & {$[0.003]$} & [0.005] \\
\hline \multirow[t]{2}{*}{ LoanDeposit } & -0.03 & 0.19 & $0.907^{* * *}$ & $0.062 * * *$ & $-0.039 * *$ \\
\hline & [0.027] & [0.147] & [0.095] & [0.009] & [0.015] \\
\hline \multirow[t]{2}{*}{ Pr.Crd.GDP } & -0.018 & 0.198 * & -0.018 & $-0.058^{* * *}$ & $-0.057^{* * *}$ \\
\hline & {$[0.023]$} & [0.109] & [0.073] & [0.009] & [0.012] \\
\hline \multirow[t]{2}{*}{ Constant } & $0.648^{* * *}$ & 1.954 & $-1.177^{* *}$ & $-0.103 *$ & $0.503^{* * *}$ \\
\hline & [0.191] & {$[1.254]$} & [0.569] & {$[0.054]$} & [0.094] \\
\hline R-squared & 0.927 & 0.753 & 0.86 & 0.654 & 0.083 \\
\hline Observations & 2200 & 1611 & 2155 & 1717 & 2338 \\
\hline \multicolumn{6}{|c|}{ CGVSCORE Equation } \\
\hline \multirow{2}{*}{$\begin{array}{c}\text { Financial } \\
\text { Performance }\end{array}$} & $0.007^{* * *}$ & $0.004^{* * *}$ & 0.001 & 0.056 & -0.008 \\
\hline & {$[0.002]$} & {$[0.001]$} & {$[0.001]$} & {$[0.039]$} & {$[0.140]$} \\
\hline \multirow[t]{2}{*}{ Size } & $0.064^{* * *}$ & $0.061^{* * *}$ & $0.059^{* * *}$ & $0.052 * * *$ & $0.057^{* * *}$ \\
\hline & {$[0.003]$} & {$[0.004]$} & [0.003] & [0.004] & [0.004] \\
\hline \multirow[t]{2}{*}{ LoanDeposit } & -0.004 & $0.044^{* * *}$ & -0.004 & -0.007 & 0.005 \\
\hline & {$[0.010]$} & {$[0.012]$} & {$[0.010]$} & [0.014] & [0.010] \\
\hline Mean_SCORE & $0.383^{* * *}$ & $0.238^{* * *}$ & $0.358^{* * *}$ & $0.314^{* * *}$ & $0.368^{* * *}$ \\
\hline (Country/Year) & {$[0.034]$} & {$[0.042]$} & {$[0.035]$} & {$[0.041]$} & {$[0.050]$} \\
\hline Mean_SCORE & $0.651^{* * *}$ & $0.758^{* * *}$ & $0.675^{* * * *}$ & $0.684^{* * *}$ & $0.666^{* * *}$ \\
\hline (Country/Industry) & [0.035] & [0.041] & [0.036] & [0.044] & [0.043] \\
\hline \multirow[t]{2}{*}{ Constant } & $-1.252 * * *$ & $-1.268^{* * *}$ & $-1.156^{* * *}$ & $-0.973^{* * *}$ & $-1.106^{* * *}$ \\
\hline & [0.066] & [0.105] & [0.062] & [0.070] & [0.092] \\
\hline R-squared & 0.669 & 0.668 & 0.662 & 0.596 & 0.673 \\
\hline Observations & 2200 & 1611 & 2155 & 1717 & 2338 \\
\hline
\end{tabular}


Table 2. Cont.

\begin{tabular}{|c|c|c|c|c|c|}
\hline \multicolumn{6}{|c|}{ Environmental Score (ENVSCORE) } \\
\hline & $\begin{array}{l}\text { Net Interest } \\
\text { Margin }\end{array}$ & Tier1 Cap_Adq & $\begin{array}{c}\text { Non } \\
\text { Performing } \\
\text { Loans }\end{array}$ & $\begin{array}{l}\text { Cost Income } \\
\text { Ratio }\end{array}$ & Excess Return \\
\hline \multicolumn{6}{|c|}{ Financial Performance Equation } \\
\hline \multirow{2}{*}{$\begin{array}{c}\text { Financial } \\
\text { Performance_lag1 }\end{array}$} & $0.921^{* * *}$ & $0.833^{* * *}$ & $0.977^{* * *}$ & $0.576^{* * *}$ & $-0.063^{* * *}$ \\
\hline & {$[0.006]$} & {$[0.014]$} & {$[0.010]$} & {$[0.020]$} & {$[0.020]$} \\
\hline \multirow[t]{2}{*}{ ENVSCORE } & $-0.145^{*}$ & 0.167 & 0.007 & $0.089^{* * *}$ & 0.068 \\
\hline & {$[0.077]$} & [0.392] & {$[0.279]$} & {$[0.025]$} & {$[0.044]$} \\
\hline \multirow[t]{2}{*}{ Size } & 0.006 & -0.004 & 0.002 & -0.001 & $-0.024 * * *$ \\
\hline & [0.016] & {$[0.084]$} & {$[0.058]$} & {$[0.005]$} & [0.009] \\
\hline \multirow[t]{2}{*}{ LoanDeposit } & -0.007 & 0.23 & $0.936^{* * *}$ & $0.057^{* * *}$ & $-0.050 * * *$ \\
\hline & {$[0.028]$} & [0.147] & {$[0.095]$} & [0.009] & [0.016] \\
\hline \multirow[t]{2}{*}{ Pr.Crd.GDP } & -0.005 & $0.264^{* *}$ & 0.019 & $-0.058^{* * *}$ & $-0.060 * * *$ \\
\hline & {$[0.021]$} & [0.103] & [0.069] & [0.007] & [0.011] \\
\hline \multirow[t]{2}{*}{ Constant } & 0.084 & 2.075 & -1.366 & $0.153 *$ & $0.740 * * *$ \\
\hline & [0.289] & [1.718] & [1.009] & [0.085] & [0.157] \\
\hline R-squared & 0.926 & 0.753 & 0.86 & 0.649 & 0.077 \\
\hline Observations & 2200 & 1611 & 2155 & 1717 & 2338 \\
\hline \multicolumn{6}{|c|}{ ENVSCORE Equation } \\
\hline \multirow{2}{*}{$\begin{array}{c}\text { Financial } \\
\text { Performance }\end{array}$} & 0.005 & $0.008^{* * *}$ & $0.004^{* * *}$ & 0.094 * & 0.121 \\
\hline & [0.003] & [0.002] & {$[0.001]$} & [0.048] & [0.147] \\
\hline \multirow[t]{2}{*}{ Size } & $0.137^{* * *}$ & $0.140^{* * *}$ & $0.137^{* * *}$ & $0.128^{* * *}$ & $0.132 * * *$ \\
\hline & {$[0.005]$} & {$[0.005]$} & {$[0.004]$} & [0.005] & {$[0.005]$} \\
\hline \multirow[t]{2}{*}{ LoanDeposit } & -0.009 & 0.003 & -0.009 & $-0.057^{* * *}$ & 0.007 \\
\hline & {$[0.014]$} & [0.017] & {$[0.014]$} & [0.018] & {$[0.014]$} \\
\hline Mean_SCORE & 0.062 & 0.048 & 0.011 & $0.243 * * *$ & 0.031 \\
\hline (Country/Year) & {$[0.045]$} & [0.048] & {$[0.042]$} & {$[0.054]$} & {$[0.042]$} \\
\hline Mean_SCORE & $0.700^{* * *}$ & $0.717^{* * *}$ & $0.676^{* * *}$ & $0.664^{* * *}$ & $0.696^{* * *}$ \\
\hline (Country/Industry) & [0.027] & [0.032] & [0.028] & {$[0.033]$} & [0.030] \\
\hline \multirow[t]{2}{*}{ Constant } & $-2.494^{* * *}$ & $-2.584^{* * *}$ & $-2.465^{* * *}$ & $-2.335^{* * *}$ & $-2.427^{* * *}$ \\
\hline & [0.088] & [0.138] & [0.082] & [0.089] & [0.106] \\
\hline R-squared & 0.588 & 0.608 & 0.592 & 0.596 & 0.578 \\
\hline Observations & 2200 & 1611 & 2155 & 1717 & 2338 \\
\hline \multicolumn{6}{|c|}{ Social Score (SOCSCORE) } \\
\hline & $\begin{array}{l}\text { Net Interest } \\
\text { Margin }\end{array}$ & Tier1 Cap_Adq & $\begin{array}{c}\text { Non } \\
\text { Performing } \\
\text { Loans }\end{array}$ & $\begin{array}{l}\text { Cost Income } \\
\text { Ratio }\end{array}$ & Excess Return \\
\hline \multicolumn{6}{|c|}{ Financial Performance Equation } \\
\hline \multirow[t]{2}{*}{$\begin{array}{c}\text { Financial } \\
\text { Performance_lag1 }\end{array}$} & $0.923^{* * *}$ & $0.830 * * *$ & $0.977^{* * *}$ & $0.582^{* * *}$ & $-0.061^{* * *}$ \\
\hline & {$[0.006]$} & [0.014] & [0.009] & {$[0.019]$} & {$[0.020]$} \\
\hline \multirow[t]{2}{*}{ SOCSCORE } & -0.084 & 0.389 & 0.046 & $0.082 * * *$ & 0.042 \\
\hline & {$[0.077]$} & {$[0.378]$} & {$[0.262]$} & {$[0.027]$} & [0.043] \\
\hline \multirow[t]{2}{*}{ Size } & -0.007 & -0.033 & -0.003 & 0.002 & $-0.018^{* *}$ \\
\hline & [0.015] & {$[0.072]$} & {$[0.048]$} & {$[0.005]$} & [0.008] \\
\hline \multirow[t]{2}{*}{ LoanDeposit } & -0.008 & 0.184 & $0.932^{* * *}$ & $0.056^{* * *}$ & $-0.049 * * *$ \\
\hline & [0.029] & {$[0.154]$} & {$[0.097]$} & [0.009] & [0.016] \\
\hline \multirow[t]{2}{*}{ Pr.Crd.GDP } & -0.004 & $0.297^{* * * *}$ & 0.023 & $-0.055^{* * *}$ & $-0.059 * * *$ \\
\hline & [0.021] & [0.109] & {$[0.072]$} & [0.007] & [0.012] \\
\hline \multirow[t]{2}{*}{ Constant } & 0.303 & 2.518 & -1.283 & 0.077 & $0.635^{* * *}$ \\
\hline & [0.259] & [1.535] & [0.811] & {$[0.076]$} & [0.132] \\
\hline R-squared & 0.926 & 0.754 & 0.86 & 0.65 & 0.079 \\
\hline Observations & 2200 & 1611 & 2155 & 1717 & 2338 \\
\hline
\end{tabular}


Table 2. Cont.

\begin{tabular}{cccccc}
\hline & \multicolumn{5}{c}{ SOCSCORE Equation } \\
Financial & $0.016^{* * *}$ & $0.008^{* * *}$ & 0.002 & $0.107^{* * *}$ & $0.403^{* * *}$ \\
Performance & {$[0.003]$} & {$[0.002]$} & {$[0.001]$} & {$[0.044]$} & {$[0.154]$} \\
& $0.119^{* * *}$ & $0.114^{* * *}$ & $0.110^{* * *}$ & $0.108^{* * *}$ & $0.114^{* * *}$ \\
Size & {$[0.004]$} & {$[0.004]$} & {$[0.004]$} & {$[0.004]$} & {$[0.005]$} \\
& -0.007 & 0.02 & -0.014 & $-0.048^{* * *}$ & $0.019^{*}$ \\
LoanDeposit & {$[0.013]$} & {$[0.015]$} & {$[0.014]$} & {$[0.017]$} & {$[0.015]$} \\
& $0.216^{* * *}$ & $0.167^{* * *}$ & $0.187^{* * *}$ & $0.292^{* * *}$ & $0.196^{* * *}$ \\
Mean_SCORE & {$[0.043]$} & {$[0.046]$} & {$[0.043]$} & {$[0.049]$} & {$[0.049]$} \\
Country/Year) & $0.694^{* * *}$ & $0.764^{* * *}$ & $0.711^{* * *}$ & $0.644^{* * *}$ & $0.680^{* * *}$ \\
Mean_SCORE & {$[0.027]$} & {$[0.029]$} & {$[0.027]$} & {$[0.034]$} & {$[0.034]$} \\
(Country/Industry) & $-2.203^{* * *}$ & $-2.239^{* * *}$ & $-2.009 * * *$ & $-1.920^{* * *}$ & $-2.149^{* * *}$ \\
Constant & {$[0.080]$} & {$[0.119]$} & {$[0.073]$} & {$[0.080]$} & {$[0.110]$} \\
R-squared & 0.593 & 0.643 & 0.595 & 0.581 & 0.465 \\
Observations & 2200 & 1611 & 2155 & 1717 & 2338 \\
\hline
\end{tabular}

In Table 3, we report the relationship with more fine-grained components of responsibility in each of these three domains with 3SLS estimations only (see Appendix A for the description of these indicators). More specifically, we have the following components of the generic governance score (CGVSCORE); board function (CGBF), board structure (CGBS), compensation policy (CGCP), vision and strategy (CGVS), and shareholder rights (CGSR). For the generic environmental score (ENVSCORE), we have emission reduction (ENER), product innovation (ENPI), and resource reduction (ENRR). For the generic social score (SOCSCORE), we have product responsibility (SOPR), community (SOCO), human rights (SOHR), diversity and opportunity (SODO), employment quality (SOEQ), health and safety (SOHS), and training and development (SOTD).

Table 3. The relationship between components of responsibility scores and financial performance. This table reports 3SLS estimations for the relationship between bank specific financial performance measures and the components of responsibility scores with CGVSCORE, ENVSCORE, and SOCSCORE. The definitions of components and all other variables are given in Appendix A. All regressions control year fixed effects. The sample period is from 2002 to 2015. Robust standard errors presented in brackets are clustered at the firm level, and ${ }^{* * *}, * *$, and * denote statistical significance at the $1 \%, 5 \%$, and $10 \%$ levels.

\begin{tabular}{|c|c|c|c|c|c|}
\hline \multicolumn{6}{|c|}{ Components of Corporate Governance Score (CGVSCORE) } \\
\hline & $\begin{array}{l}\text { CGBF (Board } \\
\text { Function) }\end{array}$ & $\begin{array}{c}\text { CGBS } \\
\text { (Board } \\
\text { Structure) }\end{array}$ & $\begin{array}{c}\text { CGCP (Compensation } \\
\text { Policy) }\end{array}$ & $\begin{array}{c}\text { CGVS } \\
\text { (Vision-Strategy }\end{array}$ & $\begin{array}{c}\text { CGSR } \\
\text { (Share-Holder } \\
\text { Rights) }\end{array}$ \\
\hline \multicolumn{6}{|c|}{ Net Interest Margin Ratio (NIM) } \\
\hline Component of & $0.124^{* * *}$ & $0.104^{* *}$ & $0.107^{* *}$ & $-0.136^{*}$ & 0.061 \\
\hline CGVSCORE & [0.043] & {$[0.045]$} & [0.043] & [0.076] & [0.064] \\
\hline R-squared & 0.925 & 0.925 & 0.925 & 0.925 & 0.925 \\
\hline \multicolumn{6}{|c|}{ Component of CGVSCORE Equation } \\
\hline NIM & 0.003 & 0.003 & 0.002 & $0.011^{* * *}$ & 0.002 \\
\hline & [0.003] & [0.003] & [0.003] & {$[0.003]$} & [0.004] \\
\hline R-squared & 0.671 & 0.656 & 0.653 & 0.547 & 0.294 \\
\hline Observations & 2200 & 2200 & 2200 & 2200 & 2200 \\
\hline \multicolumn{6}{|c|}{ Tier1 Capital Adequacy Ratio (TIER1) } \\
\hline Component of & 0.139 & $0.487^{* *}$ & 0.452 ** & 0.019 & 0.034 \\
\hline CGVSCORE & {$[0.215]$} & {$[0.228]$} & {$[0.221]$} & {$[0.375]$} & {$[0.345]$} \\
\hline R-squared & 0.748 & 0.749 & 0.748 & 0.748 & 0.748 \\
\hline
\end{tabular}


Table 3. Cont.

\begin{tabular}{|c|c|c|c|c|c|}
\hline \multicolumn{6}{|c|}{ Component of CGVSCORE Equation } \\
\hline \multirow[t]{2}{*}{ NIM } & 0.003 & 0.003 & 0.002 & $0.011^{* * *}$ & 0.002 \\
\hline & [0.003] & [0.003] & [0.003] & [0.003] & [0.004] \\
\hline R-squared & 0.671 & 0.656 & 0.653 & 0.547 & 0.294 \\
\hline Observations & 2200 & 2200 & 2200 & 2200 & 2200 \\
\hline \multicolumn{6}{|c|}{ Tier1 Capital Adequacy Ratio (TIER1) } \\
\hline Component of & 0.139 & $0.487^{* *}$ & $0.452 * *$ & 0.019 & 0.034 \\
\hline CGVSCORE & [0.215] & {$[0.228]$} & [0.221] & [0.375] & [0.345] \\
\hline R-squared & 0.748 & 0.749 & 0.748 & 0.748 & 0.748 \\
\hline \multicolumn{6}{|c|}{ Component of CGVSCORE Equation } \\
\hline \multirow[t]{2}{*}{ TIER1 } & 0.001 & $0.004^{* * *}$ & 0 & $0.008 * * *$ & 0 \\
\hline & [0.002] & {$[0.001]$} & [0.001] & [0.002] & [0.002] \\
\hline R-squared & 0.659 & 0.665 & 0.678 & 0.578 & 0.293 \\
\hline Observations & 1611 & 1611 & 1611 & 1611 & 1611 \\
\hline \multicolumn{6}{|c|}{ Non Performing Loans Ratio (NPL) } \\
\hline Component of & $0.324 * *$ & -0.104 & $0.263 *$ & -0.319 & $0.508^{* *}$ \\
\hline CGVSCORE & {$[0.144]$} & [0.155] & [0.149] & [0.267] & {$[0.208]$} \\
\hline R-squared & 0.85 & 0.85 & 0.85 & 0.849 & 0.849 \\
\hline \multicolumn{6}{|c|}{ Component of CGVSCORE Equation } \\
\hline \multirow[t]{2}{*}{ NPL } & $0.004^{* * *}$ & $0.003 * * *$ & -0.001 & 0.002 & -0.002 \\
\hline & {$[0.001]$} & {$[0.001]$} & {$[0.001]$} & {$[0.001]$} & [0.002] \\
\hline R-squared & 0.664 & 0.642 & 0.647 & 0.546 & 0.318 \\
\hline Observations & 2155 & 2155 & 2155 & 2155 & 2155 \\
\hline \multicolumn{6}{|c|}{ Cost to Income Ratio (CIR) } \\
\hline Component of & -0.029 & $-0.041 * *$ & -0.002 & $0.124 * * *$ & -0.035 \\
\hline CGVSCORE & [0.021] & [0.018] & {$[0.020]$} & {$[0.025]$} & [0.022] \\
\hline R-squared & 0.592 & 0.593 & 0.595 & 0.593 & 0.595 \\
\hline \multicolumn{6}{|c|}{ Component of CGVSCORE Equation } \\
\hline \multirow[t]{2}{*}{ CIR } & -0.041 & 0.041 & -0.047 & 0.037 & $0.305^{* * *}$ \\
\hline & [0.042] & [0.042] & {$[0.041]$} & {$[0.052]$} & {$[0.053]$} \\
\hline R-squared & 0.622 & 0.646 & 0.602 & 0.553 & 0.26 \\
\hline Observations & 1717 & 1717 & 1717 & 1717 & 1717 \\
\hline \multicolumn{6}{|c|}{ Excess Stock Return (ESR) } \\
\hline Component of & $-0.045^{*}$ & -0.028 & -0.029 & $0.089 * *$ & $-0.096^{* * *}$ \\
\hline CGVSCORE & {$[0.023]$} & [0.025] & {$[0.024]$} & {$[0.043]$} & [0.034] \\
\hline R-squared & 0.022 & 0.021 & 0.022 & 0.018 & 0.018 \\
\hline \multicolumn{6}{|c|}{ Component of CGVSCORE Equation } \\
\hline \multirow[t]{2}{*}{ ESR } & -0.136 & -0.184 & -0.021 & 0.193 & $0.366^{*}$ \\
\hline & [0.138] & [0.138] & {$[0.131]$} & [0.162] & [0.198] \\
\hline R-squared & 0.662 & 0.634 & 0.664 & 0.519 & 0.226 \\
\hline Observations & 2338 & 2338 & 2338 & 2338 & 2338 \\
\hline \multicolumn{6}{|c|}{ Components of Environmental Score (ENVSCORE) } \\
\hline & \multicolumn{2}{|c|}{$\begin{array}{l}\text { ENER (Emission } \\
\text { Reduction) }\end{array}$} & $\begin{array}{l}\text { ENPI (Produ } \\
\text { Innovation) }\end{array}$ & \multicolumn{2}{|c|}{ ENRR (Resource Reduction) } \\
\hline \multicolumn{6}{|c|}{ Net Interest Margin Ratio (NIM) } \\
\hline $\begin{array}{l}\text { Component of } \\
\text { ENVSCORE }\end{array}$ & & & -0.156 & & \\
\hline & & & [0.106] & & \\
\hline R-squared & & & 0.925 & & \\
\hline & & Tomponen & ISCORE Equa & & \\
\hline NIM & & & -0.001 & & \\
\hline & & & {$[0.004]$} & & \\
\hline R-squared & & & 0.471 & & \\
\hline Observations & & & 2200 & & \\
\hline & & ier1 Capit & acy Ratio (TI & & \\
\hline $\begin{array}{l}\text { Component of } \\
\text { ENVSCORE }\end{array}$ & & & 0.119 & & \\
\hline & & & [0.520] & & \\
\hline R-squared & & & 0.749 & & \\
\hline
\end{tabular}


Table 3. Cont.

\begin{tabular}{|c|c|c|c|c|c|c|c|}
\hline \multicolumn{8}{|c|}{ Component of ENVSCORE Equation } \\
\hline \multirow{2}{*}{\multicolumn{2}{|c|}{ TIER1 }} & \multicolumn{2}{|c|}{$0.008^{* * *}$} & \multicolumn{2}{|c|}{$0.006^{* * *}$} & \multicolumn{2}{|c|}{$0.007 * * *$} \\
\hline & & \multicolumn{2}{|c|}{$[0.002]$} & \multicolumn{2}{|c|}{$[0.002]$} & \multicolumn{2}{|c|}{$[0.002]$} \\
\hline \multirow{2}{*}{\multicolumn{2}{|c|}{$\begin{array}{c}\text { R-squared } \\
\text { Observations }\end{array}$}} & \multicolumn{2}{|c|}{0.608} & \multicolumn{2}{|c|}{0.497} & \multicolumn{2}{|c|}{0.571} \\
\hline & & \multicolumn{2}{|c|}{1611} & \multicolumn{2}{|c|}{1611} & \multicolumn{2}{|c|}{1611} \\
\hline \multicolumn{8}{|c|}{ Non Performing Loans Ratio (NPL) } \\
\hline \multirow{2}{*}{\multicolumn{2}{|c|}{ Component of ENVSCORE }} & & & -0.6 & $631 *$ & & \\
\hline & & & & {$[0.3$} & 374] & & \\
\hline R-squ & sared & & & & 85 & & \\
\hline & & & mponent o & ENVSCORE Equ & dation & & \\
\hline $\mathrm{Nl}$ & & 0.0 & & 0.00 & $5^{* * *}$ & & \\
\hline & & {$[0$.} & & {$[0$.} & $01]$ & & \\
\hline R-squ & sared & & & & 173 & & \\
\hline Observ & rations & & & & 55 & & \\
\hline & & & Cost to I & ome Ratio (CIR & & & \\
\hline Component o & f ENVSCORE & 0.07 & & 0.17 & $4 * * *$ & 0.06 & $* * *$ \\
\hline & & & & {$[0$.} & 34] & & \\
\hline R-squ & sared & & & & 589 & & \\
\hline & & & mponent o & ENVSCORE Equ & 1ation & & \\
\hline $\mathrm{Cl}$ & & & & 0.12 & $29 * *$ & & \\
\hline & & & & {$[0$.} & 52] & & \\
\hline R-squ & lared & & & & .5 & & \\
\hline Obsert & rations & & & & 17 & & \\
\hline & & & Excess $\mathrm{S}$ & ck Return (ESR) & & & \\
\hline Component o & f ENVSCORE & & & 0.1 & $11 *$ & & \\
\hline & & & & {$[0$.} & 60] & & \\
\hline R-squ & sared & & & & 02 & & \\
\hline & & & mponent o & ENVSCORE Equ & ration & & \\
\hline $\mathrm{ES}$ & & & & & 101 & & \\
\hline & & & & {$[0]$.} & 60] & & \\
\hline R-squ & lared & & & & 46 & & \\
\hline Observ & rations & & & & 38 & & \\
\hline & & & onents of & cial Score (SOC & SCORE) & & \\
\hline & $\begin{array}{c}\text { SOPR } \\
\text { (Product } \\
\text { Res-ponsibility) }\end{array}$ & $\begin{array}{l}\text { SOCO(Com } \\
\text { munity) }\end{array}$ & $\begin{array}{l}\text { SOHR } \\
\text { (Human } \\
\text { Rights) }\end{array}$ & $\begin{array}{c}\text { SODO } \\
\text { (Diversity \& } \\
\text { Opportunity) }\end{array}$ & $\begin{array}{c}\text { SOEQ } \\
\text { (Employ-ment } \\
\text { Quality) }\end{array}$ & $\begin{array}{l}\text { SOHS (Health } \\
\text { \& Safety) }\end{array}$ & $\begin{array}{c}\text { SOTD } \\
\text { (Training } \\
\text { \&Development) }\end{array}$ \\
\hline & & & Net Interes & Margin Ratio (N & IM) & & \\
\hline $\begin{array}{l}\text { Component } \\
\text { of }\end{array}$ & -0.13 & 0.027 & $-0.215^{* * *}$ & -0.064 & 0.028 & -0.066 & -0.067 \\
\hline SOCSCORE & [0.096] & [0.090] & [0.073] & [0.066] & [0.067] & [0.059] & [0.086] \\
\hline R-squared & 0.925 & 0.925 & 0.925 & 0.925 & 0.925 & 0.925 & 0.925 \\
\hline & & & mponent $\mathrm{c}$ & SOCSCORE Equ & lation & & \\
\hline NIM & $0.021^{* * *}$ & $0.009^{* * *}$ & $0.006^{* *}$ & $0.012^{* * *}$ & $0.014^{* * *}$ & $0.008^{* * *}$ & $0.013^{* * *}$ \\
\hline & [0.004] & [0.003] & [0.003] & [0.004] & [0.004] & [0.003] & [0.003] \\
\hline R-squared & 0.361 & 0.374 & 0.525 & 0.482 & 0.415 & 0.559 & 0.52 \\
\hline Observations & 2200 & 2200 & 2200 & 2200 & 2200 & 2200 & 2200 \\
\hline & & & r1 Capital & lequacy Ratio ( & IER1) & & \\
\hline $\begin{array}{l}\text { Component } \\
\text { of }\end{array}$ & -0.036 & 0.198 & 0.243 & 0.409 & 0.651 * & 0.155 & 0.059 \\
\hline SOCSCORE & [0.461] & [0.437] & [0.368] & [0.325] & [0.344] & [0.295] & [0.442] \\
\hline R-squared & 0.747 & 0.748 & 0.748 & 0.749 & 0.749 & 0.748 & 0.748 \\
\hline & & & mponent c & SOCSCORE Equ & lation & & \\
\hline TIER1 & $0.005^{* * *}$ & $0.004^{* *}$ & $0.007^{* * *}$ & $0.009 * * *$ & $0.007^{* * *}$ & $0.004^{* *}$ & $0.005^{* * *}$ \\
\hline & [0.002] & {$[0.002]$} & [0.002] & [0.002] & [0.002] & {$[0.002]$} & [0.002] \\
\hline R-squared & 0.397 & 0.405 & 0.557 & 0.52 & 0.444 & 0.606 & 0.551 \\
\hline Observations & 1611 & 1611 & 1611 & 1611 & 1611 & 1611 & 1611 \\
\hline & & & on Perform & ig Loans Ratio ( & NPL) & & \\
\hline $\begin{array}{l}\text { Component } \\
\text { of }\end{array}$ & -0.033 & $-0.524 *$ & -0.408 & 0.055 & $0.645^{* * *}$ & 0.204 & 0.248 \\
\hline SOCSCORE & [0.322] & [0.303] & [0.253] & [0.223] & [0.230] & [0.206] & [0.305] \\
\hline R-squared & 0.849 & 0.848 & 0.849 & 0.849 & 0.85 & 0.849 & 0.849 \\
\hline
\end{tabular}


Table 3. Cont.

\begin{tabular}{|c|c|c|c|c|c|c|c|}
\hline \multicolumn{8}{|c|}{ Component of SOCSCORE Equation } \\
\hline NPL & $0.004 * *$ & 0.002 & 0.001 & 0 & 0.001 & $0.003 * * *$ & 0.001 \\
\hline & {$[0.002]$} & [0.001] & [0.001] & [0.002] & [0.002] & {$[0.001]$} & {$[0.001]$} \\
\hline R-squared & 0.355 & 0.368 & 0.527 & 0.484 & 0.41 & 0.583 & 0.519 \\
\hline Observations & 2155 & 2155 & 2155 & 2155 & 2155 & 2155 & 2155 \\
\hline \multicolumn{8}{|c|}{ Cost to Income Ratio (CIR) } \\
\hline $\begin{array}{l}\text { Component } \\
\text { of }\end{array}$ & $0.067^{* *}$ & $0.071 * *$ & $0.077^{* * *}$ & 0.01 & $0.074^{* *}$ & $0.091 * * *$ & $0.117^{* * *}$ \\
\hline SOCSCORE & {$[0.028]$} & [0.029] & [0.023] & [0.024] & [0.032] & {$[0.020]$} & {$[0.030]$} \\
\hline R-squared & 0.589 & 0.587 & 0.593 & 0.593 & 0.563 & 0.593 & 0.581 \\
\hline \multicolumn{8}{|c|}{ Component of SOCSCORE Equation } \\
\hline \multirow[t]{2}{*}{ CIR } & 0.088 & 0.075 & $0.119^{* * *}$ & 0.023 & 0.032 & 0.029 & -0.023 \\
\hline & {$[0.054]$} & [0.048] & {$[0.046]$} & [0.051] & [0.053] & [0.045] & [0.052] \\
\hline R-squared & 0.379 & 0.396 & 0.531 & 0.482 & 0.311 & 0.545 & 0.508 \\
\hline Observations & 1717 & 1717 & 1717 & 1717 & 1717 & 1717 & 1717 \\
\hline \multicolumn{8}{|c|}{ Excess Stock Return (ESR) } \\
\hline $\begin{array}{l}\text { Component } \\
\text { of }\end{array}$ & -0.004 & $0.082 *$ & 0.079 * & 0.014 & -0.034 & $0.067 * *$ & 0.034 \\
\hline SOCSCORE & {$[0.053]$} & [0.049] & [0.042] & [0.036] & {$[0.038]$} & {$[0.034]$} & {$[0.050]$} \\
\hline R-squared & 0.024 & 0.015 & 0.014 & 0.021 & 0.022 & 0.018 & 0.022 \\
\hline \multicolumn{8}{|c|}{ Component of SOCSCORE Equation } \\
\hline ESR & $0.505^{* *}$ & 0.149 & $0.481^{* * *}$ & -0.145 & 0.023 & 0.103 & $0.502 * * *$ \\
\hline & [0.198] & [0.157] & [0.167] & [0.177] & [0.142] & [0.136] & [0.191] \\
\hline R-squared & 0.143 & 0.349 & 0.326 & 0.48 & 0.42 & 0.558 & 0.311 \\
\hline Observations & 2338 & 2338 & 2338 & 2338 & 2338 & 2338 & 2338 \\
\hline
\end{tabular}

In Table 2, it shows that governance had a significant effect on NIM only. Table 3 shows that banks with higher scores regarding board function, board structure and compensation policy have higher NIM, but a higher score on vision and strategy reduces margins, both supporting H1. Among those, only vision and strategy have a bidirectional relationship with banks' NIM. This is consistent with both $\mathrm{H} 1$ and $\mathrm{H} 2$. The other statistically significant positive effects of the governance components on financial performance are as follows: board structure and compensation policy on TIER1; board function and compensation policy on NPL; vision and strategy on CIR; and vision and strategy on ESR. On the other hand, there is also a significantly negative relationship between board structure and $\mathrm{CIR}$, and between board function and shareholder rights with ESR. When we test $\mathrm{H} 2$ by assessing the effects of financial performance proxies on the fine-grained governance components, we confirm the general finding in Table 2 of positive and significant effects of net margins and TIER1 on governance. Table 3 also shows that these effects are primarily based on vision and strategy for NIM and on board structure and vision and strategy for TIER1. However, we also observe the positive and significant effects of NPL on board function and board strategy, and of the CIR and ESR on shareholder rights.

Regarding our $\mathrm{H} 1$ for the ENVSCORE, recall that in Table 2 we found a significantly negative effect on NIM and a positive effect on CIR. In Table 3, we confirm the negative effect on margins with energy reduction and reduction of resource usage, and the positive effect on efficiency with product innovation in addition to the other two components. In addition, product innovation also has significantly negative (positive) effects on NPL (ESR). In terms of H2 testing the effects of the financial performance on those three components, we find strong positive effects of TIER1 and NPL on ENVSCORE with all three of its components, with the exception of NPL on resource reduction. The margin only has a marginally significant positive effect on emission reduction. With ESR and CIR, there is only a significant positive impact on product innovation.

When we examine the components of SOCSCORE in Table 3, we have strong evidence for the confirmation of the positive effect on bank efficiency. Six of the seven components of social performance have significantly positive effects on CIR, but there is only one positive bidirectional effect between the ratio and human rights. Moreover, the results confirm the positive and significant effects of NIM and TIER1 in Table 2, as both have significantly positive effects on all seven components of social 
performance. ESR has a positive and significant association with product responsibility, human rights and training and development.

The decomposition of the aggregate responsibility scores in more fine-grained indicators in Table 3 is in line with Table 2. We establish that it is usually some specific constituents that relate to the finance-responsibility nexus, not all 'ingredients' are relevant. Especially capital adequacy appears to be the driver of responsibility, confirming the available funds hypothesis. There is only scarce evidence supporting the other hypotheses.

\subsection{The Role of the Global Financial Crisis}

Due to the economic and social impact of the global financial crisis, we are also interested in how it might have affected the relationship between banks' responsibility and financial performance (see [24,33] for non-financials). Cornett et al. [15] address this for a sample of US banks and conclude that banks increase their profitability when they are being socially responsible as financial performance is positively and significantly related to responsibility. They see a role for bank size, as the biggest banks pursue more socially responsible activities than smaller banks. Further, they report large banks see a steep increase in responsibility strengths and a steep drop in responsibility concerns after the crisis. However, the sequence of the relations is not clear in their study.

To test the exploratory hypothesis regarding the impact of the global financial crisis (H3), we investigate how the crisis affects the finance-responsibility nexus. Table 1 already revealed that banks' financial performance indicators all improved significantly after the crisis and that their governance and social scores became worse. This suggests that the financial crisis had an immediate effect on banks' financial performance, which in turn affected banks' responsibility. Then, pressure from regulators to improve financial performance, especially to strengthen the capital base, as well as pressure from other stakeholders to behave responsibly, might alter the finance-responsibility nexus. However, it might also be the case that the crisis affects banks' responsibility efforts. This could be because the crisis appeals to the ethical stance and conduct of banks. Due to pressure from the public, banks might have felt an urge to improve their responsibility. Therefore, we compare pre- and post-crisis periods in order to find out whether the crisis did result in any structural change of the bank performance-responsibility nexus. Methodologically, we follow the approach used to arrive at our main results in Table 2.

To compare the pre- and post-crisis periods (2002-2007 and 2010-2015 respectively), we define a post-crisis dummy, which takes the value of 1 for post-crisis years (2010-2015) and 0 for pre-crisis years (2002-2007). Thus, stand-alone variables representing either responsibility or financial performance will show the relationships in pre-crisis years. With the interaction variables for the combined effect of the post-crisis dummy with either responsibility or financial performance, we examine how the nature of the relationships change during the post-crisis years compared to pre-crisis years. This analysis aims to detect whether the relationships between financial and non-financial performances have different characteristics before and after the crisis. If this is the case, it suggests that the crisis played significant role in banks' responsibility policies. Table 4 shows the estimation results.

Table 4 provides comparisons of the relationships between financial performance proxies and CGVSCORE between pre and post-crisis years. The results are very interesting in relation to the overall results presented for total sample period in Table 2. In the financial performance equation, CGVSCORE has a negative impact on NIM and ESR, and positively influences TIER1, NPL, and CIR. However, the effects in the post-crisis period are opposite. The coefficients of the interaction variable Post_Crisis*CGVSCORE for NIM and ESR are positive and larger than the negative coefficients of the standalone variable of CGVSCORE. This indicates that the effects of banks' governance on NIM and ESR are stronger in the post-crisis period compared to the pre-crisis period. Further, the effects of governance on TIER1, NPL, and CIR are weaker during the post crisis period relative to the pre-crisis period, when the effects of governance were significant and positive. This makes sense, as banks with stronger governance require less capital, will have less risky loans and make fewer 
costs. In Table 1, we observed that there was a reduction in banks' governance performance in the post-crisis period. The negative coefficients for the interactions in Table 5 indicate that banks with higher governance scores have lower TIER1, NPL and CIR. With respect to the financial performance equation, we find significant and positive effects for NIM, TIER1, NPL, ESR and a negative effect for $\mathrm{CIR}$ on the governance score during the pre-crisis period. These effects go in the opposite directions as is evidenced by the estimated coefficients of Post_Crisis*Financial Performance during the post-crisis period for all financial performance proxies. This supports our $\mathrm{H} 3$ and suggests that the relevance of financial performance in explaining governance performance decreased in the post-crisis period.

Table 4. 3SLS estimation for the effects of financial performance on responsibility scores with comparison between the post and pre-crisis periods in the banking industry. This table reports 3SLS estimations for simultaneous equation system of the relationship between responsibility scores and bank specific financial performance measures for the effect of crisis years with CGVSCORE, ENVSCORE, and SOCSCORE. The definitions of variables are in Appendix A. Post_Crisis is a dummy variable taking one for years after 2009, and zero for years before 2008. All regressions control year fixed effects. The sample period is from 2002 to 2015 . Robust standard errors presented in brackets are clustered at the firm level, and ${ }^{* * *}, *$, and ${ }^{*}$ denote statistical significance at the $1 \%, 5 \%$, and $10 \%$ levels.

\begin{tabular}{|c|c|c|c|c|c|}
\hline \multicolumn{6}{|c|}{ Corporate Governance (CGVSCORE) } \\
\hline & $\begin{array}{c}\text { Net Interest } \\
\text { Margin }\end{array}$ & $\begin{array}{c}\text { Tier1 } \\
\text { Cap_Adq }\end{array}$ & $\begin{array}{c}\text { Non Performing } \\
\text { Loans }\end{array}$ & $\begin{array}{c}\text { Cost Income } \\
\text { Ratio }\end{array}$ & $\begin{array}{l}\text { Excess } \\
\text { Return }\end{array}$ \\
\hline \multicolumn{6}{|c|}{ Financial Performance Equation } \\
\hline \multirow{2}{*}{$\begin{array}{c}\text { Financial } \\
\text { Performance_lag1 }\end{array}$} & $0.938^{* * *}$ & $0.886^{* * *}$ & $1.003^{* * *}$ & $0.601^{* * *}$ & -0.027 \\
\hline & [0.007] & {$[0.018]$} & {$[0.011]$} & {$[0.021]$} & [0.019] \\
\hline \multirow[t]{2}{*}{ CGVSCORE } & $-0.291 * * *$ & $23.535^{* * *}$ & $3.998 * * *$ & $0.377^{* * *}$ & $-0.150^{* * *}$ \\
\hline & [0.092] & [3.317] & {$[0.376]$} & [0.067] & {$[0.051]$} \\
\hline \multirow[t]{2}{*}{ Post_Crisis } & $-0.212 * * *$ & $13.943^{* * *}$ & $2.568 * * *$ & $0.210 * * *$ & $-0.285 * * *$ \\
\hline & [0.075] & [2.304] & [0.306] & {$[0.046]$} & {$[0.043]$} \\
\hline \multirow[t]{2}{*}{ Post_Crisis*CGVSCORI } & E $0.429 * * *$ & $-23.119 * * *$ & $-4.301 * * *$ & $-0.363 * * *$ & $0.172 * * *$ \\
\hline & [0.097] & [3.296] & [0.401] & [0.064] & {$[0.054]$} \\
\hline \multirow[t]{2}{*}{ Size } & -0.015 & -0.072 & -0.051 & $0.010^{* * *}$ & $-0.008 *$ \\
\hline & {$[0.010]$} & [0.059] & [0.033] & [0.003] & {$[0.005]$} \\
\hline \multirow[t]{2}{*}{ LoanDeposit } & -0.041 & -0.01 & $0.882 * * *$ & $0.072 * * *$ & $-0.035^{* *}$ \\
\hline & {$[0.028]$} & [0.205] & [0.113] & [0.011] & [0.015] \\
\hline \multirow[t]{2}{*}{ Pr.Crd.GDP } & 0.008 & -0.038 & -0.105 & $-0.079 * * *$ & $-0.028 * *$ \\
\hline & [0.023] & [0.149] & [0.087] & [0.010] & [0.012] \\
\hline \multirow[t]{2}{*}{ Constant } & $0.524^{* * *}$ & $-11.301^{* * *}$ & $-2.348^{* * *}$ & $-0.253^{* * *}$ & $0.490 * * *$ \\
\hline & [0.191] & [2.384] & [0.670] & [0.064] & [0.091] \\
\hline \multirow[t]{2}{*}{ R-squared } & 0.935 & 0.663 & 0.855 & 0.621 & 0.108 \\
\hline & \multicolumn{5}{|c|}{ CGVSCORE Equation } \\
\hline \multirow{2}{*}{$\begin{array}{c}\text { Financial } \\
\text { Performance }\end{array}$} & $0.023^{* * *}$ & $0.018 * *$ & $0.069 * * *$ & $-0.389 * *$ & $8.391 * * *$ \\
\hline & [0.008] & {$[0.008]$} & [0.008] & [0.155] & [1.772] \\
\hline \multirow[t]{2}{*}{ Post_Crisis } & $0.120 * * *$ & $0.256^{* *}$ & $0.180 * * *$ & $-0.168^{* *}$ & $1.549^{* * *}$ \\
\hline & [0.033] & [0.127] & {$[0.028]$} & [0.069] & {$[0.340]$} \\
\hline \multirow{2}{*}{$\begin{array}{l}\text { Post_Crisis*Financial } \\
\text { Performance }\end{array}$} & $-0.016^{* *}$ & $-0.014^{*}$ & $-0.069^{* * *}$ & $0.450 * * *$ & $-8.373^{* * *}$ \\
\hline & [0.008] & [0.008] & [0.008] & [0.149] & [1.772] \\
\hline \multirow[t]{2}{*}{ Size } & $0.063^{* * *}$ & $0.060^{* * *}$ & $0.054^{* * *}$ & $0.053^{* * *}$ & $0.076^{* * *}$ \\
\hline & [0.004] & [0.004] & {$[0.004]$} & [0.004] & [0.019] \\
\hline \multirow[t]{2}{*}{ LoanDeposit } & -0.006 & $0.052 * * *$ & 0.01 & 0.009 & -0.018 \\
\hline & [0.011] & [0.014] & [0.012] & [0.015] & [0.060] \\
\hline Mean_SCORE & $0.374^{* * *}$ & $0.236 * * *$ & $0.439 * * *$ & $0.280 * * *$ & $1.096 * * *$ \\
\hline (Country/Year) & {$[0.038]$} & {$[0.048]$} & [0.041] & [0.047] & [0.259] \\
\hline Mean_SCORE & $0.643^{* * *}$ & $0.744^{* * *}$ & $0.696^{* * *}$ & $0.695^{* * *}$ & $0.504^{* *}$ \\
\hline (Country/Industry) & [0.038] & [0.047] & [0.042] & [0.049] & [0.216] \\
\hline \multirow[t]{2}{*}{ Constant } & $-1.269 * * *$ & $-1.442 * * *$ & $-1.223^{* * *}$ & $-0.802 * * *$ & $-3.167^{* * *}$ \\
\hline & [0.076] & [0.163] & [0.073] & [0.084] & [0.574] \\
\hline R-squared & 0.664 & 0.652 & 0.608 & 0.58 & \\
\hline Observations & 1840 & 1284 & 1808 & 1464 & 1966 \\
\hline
\end{tabular}


Table 4. Cont.

\begin{tabular}{|c|c|c|c|c|c|}
\hline \multicolumn{6}{|c|}{ Environmental Score (ENVSCORE) } \\
\hline & $\begin{array}{l}\text { Net Interest } \\
\text { Margin }\end{array}$ & $\begin{array}{c}\text { Tier1 } \\
\text { Cap_Adq }\end{array}$ & $\begin{array}{c}\text { Non Performing } \\
\text { Loans }\end{array}$ & $\begin{array}{c}\text { Cost Income } \\
\text { Ratio }\end{array}$ & $\begin{array}{l}\text { Excess } \\
\text { Return }\end{array}$ \\
\hline \multicolumn{6}{|c|}{ Financial Performance Equation } \\
\hline $\begin{array}{c}\text { Financial } \\
\text { Performance lag1 }\end{array}$ & $0.934^{* * *}$ & $0.923 * * *$ & $0.976^{* * *}$ & $0.642 * * *$ & -0.021 \\
\hline & [0.007] & {$[0.074]$} & {$[0.010]$} & [0.023] & [0.019] \\
\hline ENVSCORE & $\begin{array}{c}0.668^{* * *} \\
{[0.144]}\end{array}$ & $\begin{array}{c}149.302 * * * \\
{[29.514]}\end{array}$ & $\begin{array}{c}1.921^{* * *} \\
{[0.626]}\end{array}$ & $\begin{array}{c}-0.386 * * * \\
{[0.055]}\end{array}$ & $\begin{array}{c}0.198^{* *} \\
{[0.083]}\end{array}$ \\
\hline Post_Crisis & $\begin{array}{c}0.218^{* * *} \\
{[0.078]}\end{array}$ & $\begin{array}{c}88.030^{* * *} \\
{[18.122]}\end{array}$ & $\begin{array}{c}0.701^{* *} \\
{[0.303]}\end{array}$ & $\begin{array}{c}-0.142 * * * \\
{[0.030]}\end{array}$ & $\begin{array}{c}-0.121^{* * *} \\
{[0.044]}\end{array}$ \\
\hline Post_Crisis*ENVSCORE & $\begin{array}{c}\mathrm{E}-0.540 * * * \\
{[0.123]}\end{array}$ & $\begin{array}{c}-144.383^{* * *} \\
{[28.672]}\end{array}$ & $\begin{array}{c}-1.187 * * \\
{[0.527]}\end{array}$ & $\begin{array}{c}0.324^{* * *} \\
{[0.049]}\end{array}$ & $\begin{array}{c}-0.171 \text { ** } \\
{[0.071]}\end{array}$ \\
\hline Size & $\begin{array}{l}-0.069 * * * \\
{[0.016]}\end{array}$ & $\begin{array}{c}-1.800^{* * *} \\
{[0.459]}\end{array}$ & $\begin{array}{c}-0.187^{* * *} \\
{[0.061]}\end{array}$ & $\begin{array}{c}0.038^{* * *} \\
{[0.005]}\end{array}$ & $\begin{array}{l}-0.023^{* * *} \\
{[0.008]}\end{array}$ \\
\hline LoanDeposit & $\begin{array}{l}-0.037 \\
{[0.029]}\end{array}$ & $\begin{array}{c}0.54 \\
{[0.830]}\end{array}$ & $\begin{array}{c}0.809 * * * \\
{[0.109]}\end{array}$ & $\begin{array}{c}0.062 * * * \\
{[0.011]}\end{array}$ & $\begin{array}{c}-0.036^{* *} \\
{[0.015]}\end{array}$ \\
\hline Pr.Crd.GDP & $\begin{array}{l}0.050 * * \\
{[0.024]}\end{array}$ & $\begin{array}{c}0.984 \\
{[0.608]}\end{array}$ & $\begin{array}{c}0.094 \\
{[0.086]}\end{array}$ & $\begin{array}{c}-0.065^{* * *} \\
{[0.009]}\end{array}$ & $\begin{array}{l}-0.019 \\
{[0.012]}\end{array}$ \\
\hline Constant & $\begin{array}{c}1.031^{* * *} \\
{[0.255]}\end{array}$ & $\begin{array}{c}-59.110^{* * *} \\
{[14.383]}\end{array}$ & $\begin{array}{c}1.322 \\
{[0.937]}\end{array}$ & $\begin{array}{c}-0.381 * * * \\
{[0.083]}\end{array}$ & $\begin{array}{c}0.582 * * * \\
{[0.125]}\end{array}$ \\
\hline R-squared & 0.93 & & 0.864 & 0.563 & 0.083 \\
\hline \multicolumn{6}{|c|}{ ENVSCORE Equation } \\
\hline $\begin{array}{c}\text { Financial } \\
\text { Performance }\end{array}$ & $0.031^{* * *}$ & $0.055^{* * *}$ & $-0.019^{* *}$ & $-1.758^{* * *}$ & -2.290 * \\
\hline Post_Crisis & $\begin{array}{c}{[0.010]} \\
0.248^{* * *} \\
{[0.041]}\end{array}$ & $\begin{array}{c}{[0.011]} \\
0.719 * * * \\
{[0.172]}\end{array}$ & $\begin{array}{c}{[0.009]} \\
0.149 * * \\
{[0.033]}\end{array}$ & $\begin{array}{c}{[0.226]} \\
-0.555 \text { *** } \\
{[0.101]}\end{array}$ & $\begin{array}{l}{[1.276]} \\
-0.199 \\
{[0.230]}\end{array}$ \\
\hline $\begin{array}{l}\text { Post_Crisis*Financial } \\
\text { Performance }\end{array}$ & $-0.020 * *$ & $-0.046^{* * *}$ & $0.025^{* * *}$ & $1.679^{* * *}$ & $2.289 *$ \\
\hline & {$[0.010]$} & {$[0.011]$} & {$[0.010]$} & [0.217] & [1.278] \\
\hline Size & $\begin{array}{c}0.137^{* * *} \\
{[0.005]}\end{array}$ & $\begin{array}{c}0.147^{* * *} \\
{[0.006]}\end{array}$ & $\begin{array}{c}0.135^{* * *} \\
{[0.005]}\end{array}$ & $\begin{array}{c}0.136^{* * *} \\
{[0.006]}\end{array}$ & $\begin{array}{c}0.112 * * * \\
{[0.010]}\end{array}$ \\
\hline LoanDeposit & $\begin{array}{l}-0.011 \\
{[0.015]}\end{array}$ & $\begin{array}{c}0.019 \\
{[0.020]}\end{array}$ & $\begin{array}{l}-0.021 \\
{[0.016]}\end{array}$ & $\begin{array}{c}0.012 \\
{[0.025]}\end{array}$ & $\begin{array}{l}-0.022 \\
{[0.027]}\end{array}$ \\
\hline Mean_SCORE & $0.102 * *$ & 0.005 & 0.026 & $0.333^{* * *}$ & 0.168 \\
\hline (Country/Year) & {$[0.050]$} & [0.057] & [0.049] & [0.074] & [0.108] \\
\hline Mean_SCORE & $0.709 * * *$ & $0.690 * * *$ & $0.669 * * *$ & $0.791 * * *$ & $0.795^{* * *}$ \\
\hline (Country/Industry) & [0.030] & [0.039] & [0.031] & [0.045] & [0.062] \\
\hline Constant & $\begin{array}{c}-2.600 * * * \\
{[0.101]}\end{array}$ & $\begin{array}{c}-3.308 * * * \\
{[0.226]}\end{array}$ & $\begin{array}{c}-2.388^{* * *} \\
{[0.088]}\end{array}$ & $\begin{array}{c}-1.883 * * * \\
{[0.132]}\end{array}$ & $\begin{array}{c}-1.727^{* * *} \\
{[0.343]}\end{array}$ \\
\hline R-squared & 0.584 & 0.559 & 0.592 & 0.353 & 0.002 \\
\hline Observations & 1840 & 1284 & 1808 & 1464 & 1966 \\
\hline \multicolumn{6}{|c|}{ Social Score (SOCSCORE) } \\
\hline & $\begin{array}{l}\text { Net Interest } \\
\text { Margin }\end{array}$ & $\begin{array}{c}\text { Tier1 } \\
\text { Cap_Adq }\end{array}$ & $\begin{array}{l}\text { Non Performing } \\
\text { Loans }\end{array}$ & $\begin{array}{c}\text { Cost Income } \\
\text { Ratio }\end{array}$ & $\begin{array}{l}\text { Excess } \\
\text { Return }\end{array}$ \\
\hline \multicolumn{6}{|c|}{ Financial Performance Equation } \\
\hline $\begin{array}{c}\text { Financial } \\
\text { Performance_lag1 }\end{array}$ & $0.932 * * *$ & $0.878^{* * *}$ & $1.000 * * *$ & $0.654^{* * *}$ & -0.017 \\
\hline & {$[0.007]$} & [0.018] & {$[0.012]$} & [0.025] & [0.019] \\
\hline SOCSCORE & $\begin{array}{l}0.310^{* *} \\
{[0.153]}\end{array}$ & $\begin{array}{c}-13.110 * \\
{[7.258]}\end{array}$ & $\begin{array}{c}6.209^{* * *} \\
{[0.682]}\end{array}$ & $\begin{array}{c}-0.594^{* * *} \\
{[0.080]}\end{array}$ & $\begin{array}{c}0.144 \\
{[0.088]}\end{array}$ \\
\hline Post_Crisis & $\begin{array}{c}0.135 \\
{[0.090]}\end{array}$ & $\begin{array}{l}-9.020 * \\
{[4.602]}\end{array}$ & $\begin{array}{c}2.810 * * * \\
{[0.397]}\end{array}$ & $\begin{array}{c}-0.288^{* * *} \\
{[0.045]}\end{array}$ & $\begin{array}{c}-0.128^{* *} \\
{[0.052]}\end{array}$ \\
\hline Post_Crisis*SOCSCORE & $\begin{array}{c}E-0.246^{*} \\
{[0.131]}\end{array}$ & $\begin{array}{l}13.302 * \\
{[7.036]}\end{array}$ & $\begin{array}{c}-4.949^{* * *} \\
{[0.599]}\end{array}$ & $\begin{array}{c}0.505^{* * *} \\
{[0.071]}\end{array}$ & $\begin{array}{l}-0.116 \\
{[0.076]}\end{array}$ \\
\hline Size & $\begin{array}{c}-0.040^{* * *} \\
{[0.015]}\end{array}$ & $\begin{array}{c}0.081 \\
{[0.096]}\end{array}$ & $\begin{array}{c}-0.395^{* * *} \\
{[0.059]}\end{array}$ & $\begin{array}{c}0.044^{* * *} \\
{[0.006]}\end{array}$ & $\begin{array}{c}-0.019 * * \\
{[0.007]}\end{array}$ \\
\hline LoanDeposit & $\begin{array}{l}-0.041 \\
{[0.029]}\end{array}$ & $\begin{array}{l}0.354^{*} \\
{[0.194]}\end{array}$ & $\begin{array}{c}0.548^{* * *} \\
{[0.129]}\end{array}$ & $\begin{array}{c}0.067^{* * *} \\
{[0.012]}\end{array}$ & $\begin{array}{c}-0.040 \text { ** } \\
{[0.016]}\end{array}$ \\
\hline Pr.Crd.GDP & $\begin{array}{c}0.025 \\
{[0.023]}\end{array}$ & $\begin{array}{c}0.153 \\
{[0.145]}\end{array}$ & $\begin{array}{c}0.337^{* * *} \\
{[0.100]}\end{array}$ & $\begin{array}{c}-0.064^{* * *} \\
{[0.009]}\end{array}$ & $\begin{array}{c}-0.022 * \\
{[0.013]}\end{array}$ \\
\hline Constant & $\begin{array}{c}0.660^{* * *} \\
{[0.234]}\end{array}$ & $\begin{array}{l}8.513^{* *} \\
{[3.585]}\end{array}$ & $\begin{array}{c}2.495 * * * \\
{[0.906]}\end{array}$ & $\begin{array}{c}-0.340 * * * \\
{[0.082]}\end{array}$ & $\begin{array}{c}0.523 * * * \\
{[0.111]}\end{array}$ \\
\hline R-squared & 0.934 & 0.737 & 0.819 & 0.491 & 0.084 \\
\hline
\end{tabular}


Table 4. Cont.

\begin{tabular}{cccccc}
\hline & \multicolumn{5}{c}{ SOCSCORE Equation } \\
Financial & $0.042^{* * *}$ & $0.046^{* * *}$ & $-0.031^{* * *}$ & $-1.760^{* * *}$ & $4.009^{* *}$ \\
Performance & {$[0.009]$} & {$[0.009]$} & {$[0.008]$} & {$[0.209]$} & {$[1.934]$} \\
& $0.152^{* * * *}$ & $0.588^{* * *}$ & 0.033 & $-0.669^{* * *}$ & $0.798^{* *}$ \\
Post_Crisis & {$[0.038]$} & {$[0.147]$} & {$[0.030]$} & {$[0.093]$} & {$[0.347]$} \\
& $-0.022^{* *}$ & $-0.037^{* * *}$ & $0.036^{* * *}$ & $1.692^{* * *}$ & $-4.000^{* *}$ \\
Post_Crisis*Financial & & {$[0.009]$} & {$[0.200]$} & {$[1.934]$} \\
Performance & {$[0.009]$} & {$[0.009]$} & $0.109^{* * *}$ & $0.117^{* * *}$ & $0.115^{* * *}$ \\
& $0.119^{* * *}$ & $0.117^{* * *}$ & {$[0.004]$} & {$[0.006]$} & {$[0.012]$} \\
Size & {$[0.004]$} & {$[0.005]$} & $-0.029^{*}$ & 0.034 & 0.025 \\
& -0.01 & $0.038^{* *}$ & {$[0.015]$} & {$[0.024]$} & {$[0.037]$} \\
LoanDeposit & {$[0.015]$} & {$[0.018]$} & $0.209^{* * *}$ & $0.318^{* * *}$ & 0.144 \\
& $0.261^{* * *}$ & $0.157^{* * *}$ & {$[0.048]$} & {$[0.069]$} & {$[0.119]$} \\
Mean_SCORE & {$[0.048]$} & {$[0.053]$} & $0.694^{* * *}$ & $0.800^{* * *}$ & $0.685^{* * *}$ \\
(Country/Year) & $0.699^{* * * *}$ & $0.751^{* * *}$ & {$[0.030]$} & {$[0.049]$} & {$[0.076]$} \\
Mean_SCORE & {$[0.030]$} & {$[0.035]$} & $-1.928^{* * *}$ & $-1.494^{* * *}$ & $-2.777^{* * *}$ \\
(Country/Industry) & $-2.296^{* * *}$ & $-2.779^{* * *}$ & {$[0.079]$} & {$[0.123]$} & {$[0.464]$} \\
Constant & {$[0.093]$} & {$[0.191]$} & 0.598 & 0.294 & \\
R-squared & 0.587 & 0.515 & 1808 & 1464 & 1966 \\
Observations & 1840 & 1284 & & & \\
\hline
\end{tabular}

Table 5. 3SLS estimations for the role of economic development with comparison between the post and pre-crisis periods in the banking industry. This table reports 3SLS estimations for simultaneous equation system of the relationship between responsibility scores and bank specific financial performance measures for the effect of crisis years with CGVSCORE, ENVSCORE, and SOCSCORE. Low and High income countries are determined by the bottom and top 40 percentile of GDP per Capita by year. The definitions of variables are in Appendix A. All regressions control year fixed effects. The sample period is from 2002 to 2015 . Robust standard errors presented in brackets are clustered at the firm level, and ${ }^{* * *}$, **, and * denote statistical significance at the $1 \%, 5 \%$, and $10 \%$ levels.

\begin{tabular}{|c|c|c|c|c|c|}
\hline \multicolumn{6}{|c|}{ Low Income Countries } \\
\hline & $\begin{array}{l}\text { Net Interest } \\
\text { Margin }\end{array}$ & $\begin{array}{c}\text { Tier1 } \\
\text { Cap_Adq }\end{array}$ & $\begin{array}{c}\text { Non Performing } \\
\text { Loans }\end{array}$ & $\begin{array}{l}\text { Cost Income } \\
\text { Ratio }\end{array}$ & $\begin{array}{l}\text { Excess } \\
\text { Return }\end{array}$ \\
\hline \multicolumn{6}{|c|}{ Financial Performance Equation } \\
\hline \multirow{2}{*}{$\begin{array}{c}\text { Financial } \\
\text { Performance_lag1 }\end{array}$} & $0.909 * * *$ & $0.991^{* * *}$ & $0.987^{* * *}$ & $0.695^{* * *}$ & -0.022 \\
\hline & [0.010] & {$[0.062]$} & [0.017] & [0.035] & {$[0.028]$} \\
\hline \multirow[t]{2}{*}{ CGVSCORE } & 0.454 & $120.180 * * *$ & $6.106^{* * *}$ & $-0.544^{* * *}$ & 0.12 \\
\hline & [0.358] & [16.958] & [1.763] & [0.120] & {$[0.200]$} \\
\hline \multirow[t]{2}{*}{ Post_Crisis } & -0.006 & $45.130^{* * *}$ & $2.989 * * *$ & $-0.263^{* * *}$ & $-0.216^{* *}$ \\
\hline & [0.150] & [7.508] & [0.778] & [0.075] & [0.096] \\
\hline \multirow[t]{2}{*}{ Post_Crisis*CGVSCORE } & -0.255 & $-118.805^{* * *}$ & $-6.830 * * *$ & $0.507^{* * *}$ & -0.049 \\
\hline & {$[0.345]$} & [16.911] & {$[1.708]$} & {$[0.118]$} & {$[0.192]$} \\
\hline \multicolumn{6}{|c|}{ CGVSCORE Equation } \\
\hline \multirow{2}{*}{$\begin{array}{c}\text { Financial } \\
\text { Performance }\end{array}$} & $0.055^{* * *}$ & $0.056^{* * *}$ & $0.143^{* * *}$ & $-8.659 * * *$ & $14.530 * * *$ \\
\hline & [0.018] & [0.017] & [0.022] & [1.449] & [5.093] \\
\hline \multirow[t]{2}{*}{ Post_Crisis } & $0.365^{* * *}$ & $1.279^{* * *}$ & $0.494^{* * *}$ & $-4.103^{* * *}$ & $2.886^{* * *}$ \\
\hline & [0.072] & [0.370] & [0.065] & [0.749] & [1.001] \\
\hline \multirow[t]{2}{*}{$\begin{array}{c}\text { Post_Crisis }{ }^{*} \text { Financial } \\
\text { Performance }\end{array}$} & $-0.051 * * *$ & $-0.049 * * *$ & $-0.144^{* * *}$ & $8.581^{* * *}$ & $-14.535^{* * *}$ \\
\hline & [0.019] & [0.017] & [0.022] & [1.446] & [5.098] \\
\hline Observations & 843 & 623 & 808 & 662 & 889 \\
\hline
\end{tabular}


Table 5. Cont.

\begin{tabular}{|c|c|c|c|c|c|}
\hline \multicolumn{6}{|c|}{ High Income Countries } \\
\hline & $\begin{array}{l}\text { Net Interest } \\
\text { Margin }\end{array}$ & $\begin{array}{c}\text { Tier1 } \\
\text { Cap_Adq }\end{array}$ & $\begin{array}{c}\text { Non Performing } \\
\text { Loans }\end{array}$ & $\begin{array}{l}\text { Cost Income } \\
\text { Ratio }\end{array}$ & $\begin{array}{l}\text { Excess } \\
\text { Return }\end{array}$ \\
\hline \multicolumn{6}{|c|}{ Financial Performance Equation } \\
\hline $\begin{array}{c}\text { Financial } \\
\text { Performance lag1 }\end{array}$ & $0.960 * * *$ & $0.899 * * *$ & $0.960 * * *$ & $0.439 * * *$ & $-0.108^{* * *}$ \\
\hline & {$[0.014]$} & [0.044] & [0.019] & [0.039] & [0.034] \\
\hline CGVSCORE & $\begin{array}{l}-0.065 \\
{[0.322]}\end{array}$ & $\begin{array}{c}61.871^{* * *} \\
{[15.068]}\end{array}$ & $\begin{array}{l}-3.256^{* *} \\
{[1.287]}\end{array}$ & $\begin{array}{c}0.702 * * * \\
{[0.179]}\end{array}$ & $\begin{array}{c}-0.374 * * \\
{[0.146]}\end{array}$ \\
\hline Post_Crisis & $\begin{array}{c}0.007 \\
{[0.233]}\end{array}$ & $\begin{array}{c}52.726^{* * *} \\
{[13.101]}\end{array}$ & $\begin{array}{c}-1.834^{* *} \\
{[0.883]}\end{array}$ & $\begin{array}{c}0.419^{* * * *} \\
{[0.124]}\end{array}$ & $\begin{array}{c}-0.319^{* * *} \\
{[0.107]}\end{array}$ \\
\hline Post_Crisis*CGVSCORE & $\begin{array}{c}0.071 \\
{[0.316]}\end{array}$ & $\begin{array}{c}-61.396 * * * \\
{[14.973]}\end{array}$ & $\begin{array}{l}2.632 * * \\
{[1.227]}\end{array}$ & $\begin{array}{c}-0.689 * * * \\
{[0.173]}\end{array}$ & $\begin{array}{c}0.387^{* * *} \\
{[0.144]}\end{array}$ \\
\hline \multicolumn{6}{|c|}{ CGVSCORE Equation } \\
\hline $\begin{array}{c}\text { Financial } \\
\text { Performance }\end{array}$ & 0.006 & $0.077^{* * *}$ & $-0.510 * * *$ & -0.283 & $7.183^{* * *}$ \\
\hline & {$[0.014]$} & {$[0.020]$} & [0.107] & {$[0.269]$} & [2.695] \\
\hline Post_Crisis & $\begin{array}{l}-0.021 \\
{[0.057]}\end{array}$ & $\begin{array}{c}0.538^{* *} \\
{[0.220]}\end{array}$ & $\begin{array}{c}-0.418^{* * *} \\
{[0.091]}\end{array}$ & $\begin{array}{c}-0.176^{*} \\
{[0.101]}\end{array}$ & $\begin{array}{l}0.660 \text { ** } \\
{[0.300]}\end{array}$ \\
\hline $\begin{array}{l}\text { Post_Crisis*Financial } \\
\text { Performance }\end{array}$ & -0.008 & $-0.073^{* * *}$ & $0.507^{* * *}$ & 0.323 & $-7.258^{* * *}$ \\
\hline & {$[0.014]$} & [0.020] & {$[0.107]$} & [0.249] & [2.718] \\
\hline Observations & 634 & 443 & 647 & 639 & 675 \\
\hline \multicolumn{6}{|c|}{ Low Income Countries } \\
\hline & $\begin{array}{l}\text { Net Interest } \\
\text { Margin }\end{array}$ & $\begin{array}{c}\text { Tier1 } \\
\text { Cap_Adq } \\
\end{array}$ & $\begin{array}{c}\text { Non Performing } \\
\text { Loans }\end{array}$ & $\begin{array}{l}\text { Cost Income } \\
\text { Ratio }\end{array}$ & $\begin{array}{l}\text { Excess } \\
\text { Return }\end{array}$ \\
\hline \multicolumn{6}{|c|}{ Financial Performance Equation } \\
\hline $\begin{array}{c}\text { Financial } \\
\text { Performance_lag1 }\end{array}$ & $0.932 * * *$ & $0.987^{* * *}$ & $0.983^{* * *}$ & $0.680^{* * *}$ & -0.019 \\
\hline & [0.013] & {$[0.102]$} & {$[0.016]$} & {$[0.036]$} & {$[0.028]$} \\
\hline ENVSCORE & $\begin{array}{c}-2.095^{* * *} \\
{[0.442]}\end{array}$ & $\begin{array}{c}217.732 * * * \\
{[47.081]}\end{array}$ & $\begin{array}{c}1.38 \\
{[1.697]}\end{array}$ & $\begin{array}{c}-0.558^{* * *} \\
{[0.121]}\end{array}$ & $\begin{array}{c}0.266 \\
{[0.197]}\end{array}$ \\
\hline Post_Crisis & $\begin{array}{c}-1.097^{* * *} \\
{[0.262]}\end{array}$ & $\begin{array}{c}143.826^{* * *} \\
{[32.147]}\end{array}$ & $\begin{array}{c}1.196 \\
{[0.963]}\end{array}$ & $\begin{array}{c}-0.393 * * * \\
{[0.099]}\end{array}$ & $\begin{array}{l}-0.085 \\
{[0.125]}\end{array}$ \\
\hline Post_Crisis*ENVSCORE & $\begin{array}{c}1.958 * * * \\
{[0.414]}\end{array}$ & $\begin{array}{c}-215.593 * * * \\
{[46.724]}\end{array}$ & $\begin{array}{l}-1.136 \\
{[1.583]}\end{array}$ & $\begin{array}{c}0.516^{* * *} \\
{[0.117]}\end{array}$ & $\begin{array}{l}-0.249 \\
{[0.186]}\end{array}$ \\
\hline \multicolumn{6}{|c|}{ ENVSCORE Equation } \\
\hline $\begin{array}{c}\text { Financial } \\
\text { Performance }\end{array}$ & 0.023 & $0.036^{*}$ & $0.046^{*}$ & $-5.359 * * *$ & $8.712^{* * *}$ \\
\hline & {$[0.023]$} & {$[0.021]$} & {$[0.024]$} & [1.282] & [3.372] \\
\hline Post_Crisis & $\begin{array}{c}0.284^{* * *} \\
{[0.088]}\end{array}$ & $\begin{array}{c}0.752 \\
{[0.459]}\end{array}$ & $\begin{array}{c}0.311 * * * \\
{[0.071]}\end{array}$ & $\begin{array}{c}-2.454^{* * *} \\
{[0.669]}\end{array}$ & $\begin{array}{c}1.810 * * * \\
{[0.637]}\end{array}$ \\
\hline $\begin{array}{l}\text { Post_Crisis*Financial } \\
\text { Performance }\end{array}$ & -0.018 & -0.028 & $-0.043 *$ & $5.233^{* * *}$ & $-8.694^{* * *}$ \\
\hline & {$[0.024]$} & [0.021] & {$[0.024]$} & [1.279] & {$[3.370]$} \\
\hline Observations & 843 & 623 & 808 & 662 & 889 \\
\hline \multicolumn{6}{|c|}{ High Income Countries } \\
\hline & $\begin{array}{l}\text { Net Interest } \\
\text { Margin }\end{array}$ & $\begin{array}{c}\text { Tier1 } \\
\text { Cap_Adq }\end{array}$ & $\begin{array}{l}\text { Non Performing } \\
\text { Loans }\end{array}$ & $\begin{array}{l}\text { Cost Income } \\
\text { Ratio }\end{array}$ & $\begin{array}{l}\text { Excess } \\
\text { Return }\end{array}$ \\
\hline \multicolumn{6}{|c|}{ Financial Performance Equation } \\
\hline $\begin{array}{c}\text { Financial } \\
\text { Performance_lag1 }\end{array}$ & $1.011 * * *$ & $0.811^{* * *}$ & $0.884 * * *$ & $0.551 * * *$ & $-0.096^{* * *}$ \\
\hline & [0.023] & [0.139] & [0.031] & [0.063] & [0.034] \\
\hline ENVSCORE & $\begin{array}{c}2.940 * * * \\
{[0.481]}\end{array}$ & $\begin{array}{c}136.647^{* * *} \\
{[41.337]}\end{array}$ & $\begin{array}{c}-9.208^{* * *} \\
{[2.050]}\end{array}$ & $\begin{array}{c}-1.157^{* * *} \\
{[0.230]}\end{array}$ & $\begin{array}{l}-0.101 \\
{[0.201]}\end{array}$ \\
\hline Post_Crisis & $\begin{array}{l}0.305^{* *} \\
{[0.148]}\end{array}$ & $\begin{array}{c}45.062 * * * \\
{[16.203]}\end{array}$ & $\begin{array}{c}-0.737^{*} \\
{[0.402]}\end{array}$ & $\begin{array}{c}-0.138 * * \\
{[0.055]}\end{array}$ & $\begin{array}{l}-0.068 \\
{[0.046]}\end{array}$ \\
\hline Post_Crisis*ENVSCORE & $\begin{array}{c}-1.852^{* * *} \\
{[0.318]}\end{array}$ & $\begin{array}{c}-122.264^{* * *} \\
{[37.445]}\end{array}$ & $\begin{array}{c}6.354^{* * *} \\
{[1.364]}\end{array}$ & $\begin{array}{c}0.754^{* * *} \\
{[0.155]}\end{array}$ & $\begin{array}{c}0.081 \\
{[0.129]}\end{array}$ \\
\hline
\end{tabular}


Table 5. Cont.

\begin{tabular}{|c|c|c|c|c|c|}
\hline \multicolumn{6}{|c|}{ ENVSCORE Equation } \\
\hline Financial & -0.01 & $0.187^{* * *}$ & $-0.684^{* * *}$ & $-1.386^{* * *}$ & $1.611 *$ \\
\hline & {$[0.015]$} & {$[0.032]$} & [0.117] & [0.324] & {$[0.830]$} \\
\hline Post_Crisis & $\begin{array}{l}0.143^{* *} \\
{[0.063]}\end{array}$ & $\begin{array}{c}1.875^{* * *} \\
{[0.352]}\end{array}$ & $\begin{array}{c}-0.398^{* * *} \\
{[0.107]}\end{array}$ & $\begin{array}{c}-0.365^{* * *} \\
{[0.127]}\end{array}$ & $\begin{array}{c}0.322 * * * \\
{[0.100]}\end{array}$ \\
\hline $\begin{array}{l}\text { Post_Crisis*Financial } \\
\text { Performance }\end{array}$ & 0 & $-0.176^{* * *}$ & $0.688^{* * *}$ & $1.284^{* * *}$ & $-1.621 *$ \\
\hline Observations & $\begin{array}{c}{[0.015]} \\
634\end{array}$ & $\begin{array}{c}{[0.032]} \\
443\end{array}$ & $\begin{array}{c}{[0.117]} \\
647\end{array}$ & $\begin{array}{c}{[0.295]} \\
639\end{array}$ & $\begin{array}{c}{[0.830]} \\
675\end{array}$ \\
\hline \multicolumn{6}{|c|}{ Low Income Countries } \\
\hline & $\begin{array}{l}\text { Net Interest } \\
\text { Margin }\end{array}$ & $\begin{array}{c}\text { Tier1 } \\
\text { Cap_Adq }\end{array}$ & $\begin{array}{c}\text { Non } \\
\text { Performing } \\
\text { Loans }\end{array}$ & $\begin{array}{c}\text { Cost Income } \\
\text { Ratio }\end{array}$ & $\begin{array}{l}\text { Excess } \\
\text { Return }\end{array}$ \\
\hline \multicolumn{6}{|c|}{ Financial Performance Equation } \\
\hline $\begin{array}{c}\text { Financial } \\
\text { Performance lag1 }\end{array}$ & $0.919 * * *$ & $1.174^{* *}$ & $0.985 * * *$ & $0.704^{* * *}$ & -0.015 \\
\hline & {$[0.011]$} & [0.545] & {$[0.016]$} & [0.063] & [0.029] \\
\hline SOCSCORE & $\begin{array}{c}-0.940 * \\
{[0.494]}\end{array}$ & $\begin{array}{l}-554.809 \\
{[736.447]}\end{array}$ & $\begin{array}{c}1.334 \\
{[1.999]}\end{array}$ & $\begin{array}{c}-1.755^{* * *} \\
{[0.345]}\end{array}$ & $\begin{array}{c}0.34 \\
{[0.266]}\end{array}$ \\
\hline Post_Crisis & $\begin{array}{c}-0.594^{*} \\
{[0.315]}\end{array}$ & $\begin{array}{l}-456.096 \\
{[601.733]}\end{array}$ & $\begin{array}{l}1.446 \\
{[1.255]}\end{array}$ & $\begin{array}{c}-1.257^{* * *} \\
{[0.266]}\end{array}$ & $\begin{array}{c}0.007 \\
{[0.184]}\end{array}$ \\
\hline Post_Crisis*SOCSCORE & $\begin{array}{l}0.860^{*} \\
{[0.457]}\end{array}$ & $\begin{array}{l}550.038 \\
{[729.201]}\end{array}$ & $\begin{array}{c}-1.31 \\
{[1.869]}\end{array}$ & $\begin{array}{c}1.634^{* * *} \\
{[0.330]}\end{array}$ & $\begin{array}{l}-0.339 \\
{[0.249]}\end{array}$ \\
\hline \multicolumn{6}{|c|}{ SOCSCORE Equation } \\
\hline $\begin{array}{c}\text { Financial } \\
\text { Performance }\end{array}$ & 0.016 & 0.037 * & 0.026 & $-3.072^{* * *}$ & $11.902 * *$ \\
\hline Post_Crisis & $\begin{array}{c}{[0.021]} \\
0.173^{* *} \\
{[0.081]}\end{array}$ & $\begin{array}{c}{[0.019]} \\
0.578 \\
{[0.408]}\end{array}$ & $\begin{array}{c}{[0.022]} \\
0.200 * * * \\
{[0.064]}\end{array}$ & $\begin{array}{c}{[1.004]} \\
-1.363 * * \\
{[0.529]}\end{array}$ & $\begin{array}{l}{[4.839]} \\
2.320^{* *} \\
{[0.929]}\end{array}$ \\
\hline $\begin{array}{l}\text { Post_Crisis*Financial } \\
\text { Performance }\end{array}$ & -0.002 & -0.025 & -0.021 & $2.888^{* * *}$ & $-11.904^{* *}$ \\
\hline Observations & $\begin{array}{c}{[0.022]} \\
843\end{array}$ & $\begin{array}{c}{[0.019]} \\
623\end{array}$ & $\begin{array}{c}{[0.022]} \\
808\end{array}$ & $\begin{array}{c}{[1.005]} \\
662\end{array}$ & $\begin{array}{c}{[4.838]} \\
889\end{array}$ \\
\hline \multicolumn{6}{|c|}{ High Income Countries } \\
\hline & $\begin{array}{l}\text { Net Interest } \\
\text { Margin }\end{array}$ & $\begin{array}{c}\text { Tier1 } \\
\text { Cap_Adq }\end{array}$ & $\begin{array}{c}\text { Non } \\
\text { Performing } \\
\text { Loans }\end{array}$ & $\begin{array}{c}\text { Cost Income } \\
\text { Ratio }\end{array}$ & $\begin{array}{l}\text { Excess } \\
\text { Return }\end{array}$ \\
\hline \multicolumn{6}{|c|}{ Financial Performance Equation } \\
\hline $\begin{array}{c}\text { Financial } \\
\text { Performance_lag1 }\end{array}$ & $0.984^{* * *}$ & $0.814^{* * *}$ & $0.853 * * *$ & $0.504^{* * *}$ & $-0.100 * * *$ \\
\hline SOCSCORE & $\begin{array}{c}{[0.019]} \\
2.172 * * * \\
{[0.420]}\end{array}$ & $\begin{array}{c}{[0.152]} \\
163.573^{* * *} \\
{[52.539]}\end{array}$ & $\begin{array}{c}{[0.037]} \\
-11.941 * * * \\
{[2.134]}\end{array}$ & $\begin{array}{c}{[0.050]} \\
-0.848^{* * *} \\
{[0.187]}\end{array}$ & $\begin{array}{c}{[0.035]} \\
0.05 \\
{[0.178]}\end{array}$ \\
\hline Post_Crisis & $\begin{array}{c}0.564 * * * \\
{[0.160]}\end{array}$ & $\begin{array}{l}43.195^{* *} \\
{[16.818]}\end{array}$ & $\begin{array}{l}-2.271^{* * *} \\
{[0.646]}\end{array}$ & $\begin{array}{c}-0.257^{* * *} \\
{[0.064]}\end{array}$ & $\begin{array}{l}-0.091 \\
{[0.061]}\end{array}$ \\
\hline Post_Crisis*SOCSCORE & $\begin{array}{c}-1.421 * * * \\
{[0.299]}\end{array}$ & $\begin{array}{c}-146.049^{* * *} \\
{[47.525]}\end{array}$ & $\begin{array}{c}7.418 * * * \\
{[1.490]}\end{array}$ & $\begin{array}{c}0.547^{* * *} \\
{[0.134]}\end{array}$ & $\begin{array}{c}0.074 \\
{[0.123]}\end{array}$ \\
\hline \multicolumn{6}{|c|}{ SOCSCORE Equation } \\
\hline $\begin{array}{c}\text { Financial } \\
\text { Performance }\end{array}$ & 0.003 & $0.142 * * *$ & $-0.500^{* * *}$ & $-1.383^{* * *}$ & 0.593 \\
\hline Post_Crisis & $\begin{array}{c}{[0.013]} \\
0.028 \\
{[0.058]}\end{array}$ & $\begin{array}{c}{[0.025]} \\
1.442 * * * \\
{[0.277]}\end{array}$ & $\begin{array}{c}{[0.096]} \\
-0.371 * * * \\
{[0.090]}\end{array}$ & $\begin{array}{c}{[0.276]} \\
-0.494 * * * \\
{[0.108]}\end{array}$ & $\begin{array}{c}{[0.473]} \\
0.078 \\
{[0.059]}\end{array}$ \\
\hline $\begin{array}{l}\text { Post_Crisis*Financial } \\
\text { Performance }\end{array}$ & -0.005 & $-0.130^{* * *}$ & $0.496^{* * *}$ & $1.215^{* * *}$ & -0.564 \\
\hline Observations & $\begin{array}{c}{[0.014]} \\
634\end{array}$ & $\begin{array}{c}{[0.025]} \\
443\end{array}$ & $\begin{array}{c}{[0.096]} \\
647\end{array}$ & $\begin{array}{c}{[0.256]} \\
639\end{array}$ & $\begin{array}{c}{[0.474]} \\
675\end{array}$ \\
\hline
\end{tabular}

Table 4 reports comparisons of the relationships between financial performance proxies and ENVSCORE between pre- and post-crisis years. The results for the financial performance equations 
show that the effects of banks' environmental performance on NIM, TIER1, NPL, and ESR are positive and those on CIR are negative during the pre-crisis period. However, the estimated coefficients of the interaction variables (Post_Crisis*ENVSCORE) with opposite signs in relation to the standalone variable of ENVSCORE indicate that the positive effects of NIM, TIER1, NPL, and ESR and the negative effects of CIR are weakened during the post-crisis period compared to the pre-crisis period. With respect to the environmental performance equation, ENVSCORE is affected by NIM and TIER1 (NPL, CIR, and ESR) positively (negatively) during the pre-crisis period, and those effects decrease in the post-crisis period compared to the pre-crisis years.

Table 4 also provides the comparisons between pre- and post-crisis years of the relationships between financial performance and SOCSCORE. The results for the financial performance equation show that the effects of banks' social responsibility on NIM and NPL (TIER1 and CIR) are positive (negative) during pre-crisis period. These effects are less positive (negative) during the post-crisis period compared to the pre-crisis one. Interestingly, we do not observe any significant effects of social performance on ESR in either the pre- or post-crisis period. Apart from ESR, the results for the SOCSCORE equation is very similar to the results we observe for ENVSCORE. Social performance is affected by NIM, TIER1 and ESR (NPL and CIR) significantly and positively (negatively) during the pre-crisis period, and these effects turn out to be exactly opposite during the post-crisis period relative to the pre-crisis.

Thus, as to the role of the global financial crisis, we establish that differences in bank responsibility drive the changes in the ways in which financial and responsibility performance interact in the post-crisis period. Banks' responsibility fell in the wake of the crisis and has not reverted in the period studied afterwards. We also find that the finance-responsibility nexus weakened compared to the years before the global financial crisis. This could be the case because before the crisis, it was predominantly for strategic reasons that banks engaged with responsibility. After the crisis, regulators and supervisors pressed banks to improve their financial performance.

\subsection{The Role of Economic and Institutional Development}

Hypothesis 4 tests whether and how economic development and institutions condition the finance-responsibility nexus and the role of the crisis therein. To this extent, we classify our sample countries along four different lines: 1. per capita income, 2. legal origin, 3. single versus multiple bank supervisors, and 4. central bank versus other agencies as banking supervisor (see Appendix B for the country groups). We report the results from the univariate comparisons in Appendix E, where we report mean values and two sets of comparisons for variables of banks' responsibility and financial performances for: (1) the differences in mean values of variables between the pre- and post-crisis periods for two group of countries, and (2) the differences in mean values of variables between two group of countries for all sample period along with pre and post-crisis periods. First, we examine the comparisons for low and high-income countries. Banks' governance is significantly improved in low-income countries (Low) while both governance and social performance deteriorated in high income countries (High) during the post crisis period relative to pre-crisis period. In Low for all three periods, banks' governance is significantly lower, even with improvement in governance, and environmental and social performance are significantly higher, except in pre-crisis period, than those in High. For banks' financial performance variables, all have improved during the post-crisis relative to pre-crisis period in both Low, except excess stock return, and High. The NIM (TIER1) in Low is significantly lower (higher, but not significant) than that in High before the crisis, but higher (lower) afterwards. All other financial performance variables in Low are significantly higher than those in High. The exception is ESR during the post-crisis period, when there is no significant difference between two country groups. The comparison regarding the legal origin of the countries where banks operate yields very interesting observations. Even though the governance and the environment scores of banks in civil law countries (Civil) increased after the crisis, and the governance and social score of banks in common law countries (Common) decreased, banks in Civil have significantly lower governance and 
higher environment and social performance (not significant before the crisis) than those in Common. All financial performance of banks in both Civil (except ESR) and Common improved during the post-crisis period from the levels in pre-crisis period. NIM and TIER1 (NPL and CIR) in Civil are significantly lower (higher), than those in Common. There was a significant difference in ESR in favor of banks in Civil before the crisis, but then it disappeared after the crisis. For the comparisons between countries with single and multiple supervisory authorities (Single vs. Multiple), we find that, after the crisis, banks experienced significant decrease with social scores in Single and with both governance and social scores in Multiple. Environment performance is significantly higher in Multiple during the post-crisis period relative pre-crisis. However, banks' governance is lower and environment and social scores are higher in all three periods for Single than those for Multiple. All financial performance of banks in both Single and Multiple (except ESR) improved during the post-crisis period from the levels in pre-crisis period. As in the comparisons between Civil and Common, NIM and TIER1 (NPL and CIR) in Single are significantly lower (higher), than those in Multiple. The significant difference in ESR before crisis in favor of banks in Single disappeared after the crisis. Banks experienced significant decrease with governance and environment scores and with governance only after the crisis in countries with supervision performed by outside of central bank (Non CB) and by central bank (CB), respectively. Environment performance is significantly higher in $\mathrm{CB}$ during the post-crisis period relative pre-crisis. Comparisons of Non CB and CB reveal that banks' governance is significantly lower, and environment and social scores are significantly higher in all three periods, except post crisis, for Non CB than those for CB. In terms of financial performances, NIM and TIER1 (NPL and CIR) in Non CB are significantly lower (higher), than those in CB. There is a significant difference in ESR after the crisis in favor of Non CB.)

Given the aim of this study, we are especially interested if and how income and institutional features of the country sample relate to our results regarding the finance-responsibility nexus. These factors turned out to be relevant in previous studies $[18,23,24]$. To find out if they also apply for the banking industry, we rely on the same model as used in the previous subsection but we now explicitly account for different types of development, i.e., per capita income (low versus high-income countries, Table 5, institutional development (civil law countries versus common law countries, Table 6, and the organization of bank supervision (countries with single bank supervisory authorities versus those with multiple ones, Table 7; countries with banking supervision conducted by the central bank versus those where agencies outside the central bank conduct this supervision, Table 8 .

Table 5 compares the finance-responsibility nexus along the governance, environment and social performance for low- and high-income countries (i.e., countries, which are in the bottom and top 40 percentile of GDP per capita). We provide the comparison of the relationships between financial performance proxies and CGVSCORE between pre and post-crisis years. It shows that governance improves TIER1 for banks in both low and high-income countries during the pre-crisis period. It improves efficiency (CIR) for low-income country banks but not for high-income country ones. It reduces NPL in high-income country banks, but increases NPL for low-income country banks. Better financial performance in general improves governance, apart from NPL in low-income countries. Further, the crisis has had an important role, as there is a sign switch regarding the finance-responsibility nexus. After the crisis (Post_Crisis*CGVSCORE), the impact of good governance on TIER1 decreases with banks in both low- and high-income countries relative to pre-crisis period. However, banks with relatively high governance scores reduce their NPL, but increase CIR in low-income countries. The effects of governance on these two ratios decreases in high income countries. With respect to the effects of financial performance on governance, we observe that the effects are becoming smaller for all financial variables during the post-crisis period in low-income countries. However, we establish that TIER1 and ESR reduce governance for high-income countries banks. We also find that financial performance and environmental performance significantly interact regarding most financial indicators. Here too, we find that the crisis had an impact and we detect some differences between banks in low and those in high-income countries: negative (positive) impact of ENVSCORE on NIM during 
the pre-crisis period decreases for low (high) income countries. The positive effect of environmental performance on TIER1 decreases during the post-crisis period in both groups of countries. The other difference between two types of countries is that the crisis mitigates the positive (negative) effect of NPL on environmental performance during the pre-crisis period. For the relationships between social and financial performance, it seems that the relationships are in general quite similar compared to environmental performance, apart for banks in low-income countries where they in general are weaker or absent. As such, we conclude that per capita income is relevant for the finance-responsibility nexus in relation to governance and social performance, but not with environmental performance.

Table 6 compares the finance-responsibility nexus and the role of the crisis between civil law countries (Civil) and common law countries (Common). The differences between two groups of countries are as follows: during the pre-crisis period, CGVSCORE improves TIER1 and CIR for banks in Civil, but not for those in Common (CIR increases), and reduces NIM for banks in Common, but has no effects on banks in Civil. The effects of financial performance are similar, apart for TIER, which reduces (increases) governance in Civil (Common). When we examine the effects during the post-crisis period, (Post_Crisis ${ }^{*}$ CGVSCORE), we observe smaller effects of CGVSCORE on CIR with the opposite direction in civil and common law countries. The impact of good governance on TIER1 decreases with banks in Civil only; on NIM it increases in both Civil and Common. With respect to the effects of financial performance on governance after the crisis, the major difference in both groups of countries is that TIER1 improves governance in Civil but worsens it in Common. There is a negative (positive) impact of ENVSCORE on NIM during the pre-crisis period for banks in Civil (Common), and these opposite impacts become less during the post-crisis period in both groups. The positive effect of environmental performance on TIER1 decreases during the post-crisis period in both groups of countries. The other difference between the two types of countries is that the crisis mitigates the positive effect of NPL on environmental performance in civil law countries. More importantly, we observe simultaneous significant effects between financial performance and ENVSCORE for both the pre and post-crisis period specifically for banks in common law countries. For the relationships between social and financial performance, we find that there is no effect of financial performance on SOCSCORE, except a marginal effect for ESR, both before and after the crisis with banks in Civil. Social performance does influence finance though, with the crisis having a mitigating effect. In Common, we witness two-way interaction next to a significant crisis effect. Better financial performance, except with NIM, in the post-crisis period also changes the effects on social performance opposite to the effects during pre-crisis period. In all, it shows that the division of our sample along Common and Civil is relevant as the two have a different finance-performance nexus and the financial crisis did have a different impact. These results confirm the findings of $[23,24]$, who both suggested such differences. However, as we use more detailed finance and responsibility indicators, we have expanded their original insights. Further, we show that there is support for the probing hypothesis about a different finance-responsibility nexus in relation to the legal system.

Table 7 investigates whether the organization of banking supervision matters for the finance-responsibility nexus in the banking industry in relation to the global financial crisis [18]. We first compare countries with a single bank supervisory authority (Single) with those having multiple supervisory authorities (Multiple). Regarding governance, we find that in Single, governance significantly influences TIER1, but that better capitalized banks do not have better governance. When there are multiple bank supervisors, better governance does not affect TIER1, but banks with more capital have better governance. We also find a different effect of the crisis: for banks in Single, the crisis did change how governance influences TIER1 but it did not affect the role of governance in relation to capital adequacy. For banks in Multiple, the crisis did not affect the lack of impact of governance on capital but did so regarding how capital adequacy affects governance. For environmental and social performance, we witness asymmetries between two the types of supervision, in particular the effects of responsibility on NIM, the effects of TIER1 on responsibility, and opposite relationships between responsibility and NPL. In general, the division of banks 
along single or multiple supervision seems to play a moderate role regarding the finance-responsibility nexus for NIM, TIER1 and NPL and the role of the financial crisis.

Table 8 shows the comparison of the nexus in relation to the crisis in countries with bank supervision conducted by agencies outside the central bank (Non CB) and in those where the central bank acts as the banking supervisor (CB). With banks' governance, the major difference between the two sets of countries is the relationship between CGVSCORE and TIER1. The impact of governance on TIER1 and the reverse impact are totally opposite for the two groups. While the relationship is negative for Non CB and positive for $\mathrm{CB}$ before the crisis, those effects become smaller after the crisis. The other difference is that the relationship between CIR and governance is insignificant for banks in Non CB, but the impact of governance is important in both pre and post crisis periods in CB. For environmental and social performance, it shows that for banks in $\mathrm{CB}$ the impact of financial performance on environmental and social performances are more pronounced before and after the crisis than for banks in Non CB, with ESR being the exception (the relationships between ESR and responsibility are also significant in Non CB). In all, there is evidence to conclude that there are differences in the finance-responsibility nexus for banks supervised by either the central bank or by agencies outside the central bank.

In all, Tables 5-8 reveal that changes in responsibility policies of the banks are behind the changes in the ways in which financial and responsibility performance interact in the post-crisis period. In turn, these seem to be conditioned by economic development, legal origin and organization of bank regulation and supervision. In general, we observe that the crisis weakened the finance-responsibility nexus. This could be the case because before the crisis, it was predominantly for strategic reasons that banks engaged with responsibility. After the crisis, regulators and supervisors pressed banks to improve their financial performance, which seems to have affected their investments in CSR. As mentioned before, there is no grounding theoretical framework at this stage, which offers an encompassing explanation for the findings of our exploratory analysis and we leave this for further research.

Table 6. 3SLS estimation for the role of legal origin with comparison between the post and pre-crisis periods in the banking industry. This table reports 3SLS estimations for simultaneous equation system of the relationship between responsibility scores and bank specific financial performance measures for the effect of crisis years with CGVSCORE, ENVSCORE, and SOCSCORE. The definitions of variables are in Appendix A. All regressions control year fixed effects. The sample period is from 2002 to 2015. Robust standard errors presented in brackets are clustered at the firm level, and ${ }^{* * *}, * *$, and * denote statistical significance at the $1 \%, 5 \%$, and $10 \%$ levels.

\begin{tabular}{|c|c|c|c|c|c|}
\hline \multicolumn{6}{|c|}{ Civil Law Countries } \\
\hline & $\begin{array}{l}\text { Net Interest } \\
\text { Margin }\end{array}$ & $\begin{array}{c}\text { Tier1 } \\
\text { Cap_Adq }\end{array}$ & $\begin{array}{c}\text { Non Performing } \\
\text { Loans }\end{array}$ & $\begin{array}{c}\text { Cost Income } \\
\text { Ratio }\end{array}$ & $\begin{array}{l}\text { Excess } \\
\text { Return }\end{array}$ \\
\hline \multicolumn{6}{|c|}{ Financial Performance Equation } \\
\hline $\begin{array}{c}\text { Financial } \\
\text { Performance lag1 }\end{array}$ & $0.896^{* * *}$ & $0.856^{* * *}$ & $1.022 * * *$ & $0.497 * * *$ & 0.019 \\
\hline & {$[0.010]$} & [0.033] & [0.017] & [0.043] & {$[0.027]$} \\
\hline CGVSCORE & $\begin{array}{l}-0.313 \\
{[0.198]}\end{array}$ & $\begin{array}{c}111.699 * * * \\
{[33.893]}\end{array}$ & $\begin{array}{c}7.172 * * * \\
{[1.276]}\end{array}$ & $\begin{array}{c}-0.843 * * * \\
{[0.251]}\end{array}$ & $\begin{array}{l}-0.061 \\
{[0.136]}\end{array}$ \\
\hline Post_Crisis & $\begin{array}{l}-0.066 \\
{[0.094]}\end{array}$ & $\begin{array}{l}1.786 \\
{[3.745]}\end{array}$ & $\begin{array}{c}1.905^{* * *} \\
{[0.537]}\end{array}$ & $\begin{array}{c}-0.385^{* * *} \\
{[0.090]}\end{array}$ & $\begin{array}{c}-0.421^{* * *} \\
{[0.065]}\end{array}$ \\
\hline Post_Crisis*CGVSCORE & $\begin{array}{l}0.337^{*} \\
{[0.179]}\end{array}$ & $\begin{array}{c}-110.130^{* * *} \\
{[33.662]}\end{array}$ & $\begin{array}{c}-6.395^{* * *} \\
{[1.147]}\end{array}$ & $\begin{array}{c}0.774^{* * *} \\
{[0.240]}\end{array}$ & $\begin{array}{c}0.079 \\
{[0.122]}\end{array}$ \\
\hline \multicolumn{6}{|c|}{ CGVSCORE Equation } \\
\hline $\begin{array}{c}\text { Financial } \\
\text { Performance }\end{array}$ & $0.025^{* *}$ & $-0.032 *$ & $0.069^{* * *}$ & $-2.351^{* * *}$ & $12.668^{* *}$ \\
\hline & {$[0.011]$} & {$[0.018]$} & {$[0.011]$} & {$[0.506]$} & [6.409] \\
\hline Post_Crisis & $\begin{array}{c}0.198^{* * *} \\
{[0.044]}\end{array}$ & $\begin{array}{l}-0.939 \\
{[0.618]}\end{array}$ & $\begin{array}{c}0.417^{* * *} \\
{[0.054]}\end{array}$ & $\begin{array}{c}-1.415^{* * *} \\
{[0.365]}\end{array}$ & $\begin{array}{l}4.667^{* *} \\
{[2.297]}\end{array}$ \\
\hline $\begin{array}{l}\text { Post_Crisis*Financial } \\
\text { Performance }\end{array}$ & -0.014 & $0.037^{* *}$ & $-0.069 * * *$ & $2.289 * * *$ & $-12.637 * *$ \\
\hline & {$[0.011]$} & {$[0.018]$} & {$[0.011]$} & {$[0.496]$} & [6.403] \\
\hline Observations & 939 & 669 & 584 & 589 & 1004 \\
\hline
\end{tabular}


Table 6. Cont.

\begin{tabular}{|c|c|c|c|c|c|}
\hline \multicolumn{6}{|c|}{ Common Law Countries } \\
\hline & $\begin{array}{l}\text { Net Interest } \\
\text { Margin }\end{array}$ & $\begin{array}{c}\text { Tier1 } \\
\text { Cap_Adq }\end{array}$ & $\begin{array}{c}\text { Non Performing } \\
\text { Loans }\end{array}$ & $\begin{array}{c}\text { Cost Income } \\
\text { Ratio }\end{array}$ & $\begin{array}{l}\text { Excess } \\
\text { Return }\end{array}$ \\
\hline \multicolumn{6}{|c|}{ Financial Performance Equation } \\
\hline $\begin{array}{c}\text { Financial } \\
\text { Performance_lag1 }\end{array}$ & $0.970 * * *$ & $1.061^{* * *}$ & $0.936^{* * *}$ & $0.666^{* * *}$ & $-0.081^{* * *}$ \\
\hline & {$[0.011]$} & {$[0.259]$} & {$[0.015]$} & {$[0.027]$} & {$[0.028]$} \\
\hline CGVSCORE & $\begin{array}{c}-1.143 * * * \\
{[0.381]}\end{array}$ & $\begin{array}{l}-464.702 \\
{[308.408]}\end{array}$ & $\begin{array}{c}5.895^{* * *} \\
{[1.076]}\end{array}$ & $\begin{array}{c}0.413 * * * \\
{[0.111]}\end{array}$ & $\begin{array}{c}0.159 \\
{[0.178]}\end{array}$ \\
\hline Post_Crisis & $\begin{array}{c}-0.697^{* * *} \\
{[0.251]}\end{array}$ & $\begin{array}{l}-346.834 \\
{[230.590]}\end{array}$ & $\begin{array}{c}3.929 * * * \\
{[0.722]}\end{array}$ & $\begin{array}{c}0.283 * * * \\
{[0.077]}\end{array}$ & $\begin{array}{c}0.014 \\
{[0.118]}\end{array}$ \\
\hline Post_Crisis*CGVSCORE & $\begin{array}{c}1.079 * * * \\
{[0.348]}\end{array}$ & $\begin{array}{c}454.273 \\
{[301.388]}\end{array}$ & $\begin{array}{c}-5.448^{* * *} \\
{[0.996]}\end{array}$ & $\begin{array}{c}-0.369^{* * *} \\
{[0.104]}\end{array}$ & $\begin{array}{l}-0.125 \\
{[0.163]}\end{array}$ \\
\hline \multicolumn{6}{|c|}{ CGVSCORE Equation } \\
\hline $\begin{array}{c}\text { Financial } \\
\text { Performance }\end{array}$ & $0.030 * *$ & $0.101 * * *$ & $0.078^{* *}$ & $-0.601 * * *$ & $6.096^{* * *}$ \\
\hline Post_Crisis & $\begin{array}{c}{[0.013]} \\
0.084 \\
{[0.052]}\end{array}$ & $\begin{array}{c}{[0.027]} \\
0.784^{* * *} \\
{[0.242]}\end{array}$ & $\begin{array}{c}{[0.031]} \\
0.062 \\
{[0.041]}\end{array}$ & $\begin{array}{c}{[0.164]} \\
-0.274^{* * *} \\
{[0.066]}\end{array}$ & $\begin{array}{l}{[2.344]} \\
0.476^{* *} \\
{[0.208]}\end{array}$ \\
\hline $\begin{array}{l}\text { Post_Crisis*Financial } \\
\text { Performance }\end{array}$ & $-0.028^{* *}$ & $-0.097^{* * *}$ & $-0.078 * *$ & $0.661^{* * *}$ & $-6.081^{* * *}$ \\
\hline & [0.013] & [0.027] & [0.031] & [0.157] & [2.341] \\
\hline Observations & 887 & 644 & 912 & 858 & 945 \\
\hline \multicolumn{6}{|c|}{ Civil Law Countries } \\
\hline & $\begin{array}{l}\text { Net Interest } \\
\text { Margin }\end{array}$ & $\begin{array}{c}\text { Tier1 } \\
\text { Cap_Adq }\end{array}$ & $\begin{array}{c}\text { Non Performing } \\
\text { Loans }\end{array}$ & $\begin{array}{c}\text { Cost Income } \\
\text { Ratio }\end{array}$ & $\begin{array}{l}\text { Excess } \\
\text { Return }\end{array}$ \\
\hline \multicolumn{6}{|c|}{ Financial Performance Equation } \\
\hline $\begin{array}{c}\text { Financial } \\
\text { Performance_lag1 }\end{array}$ & $0.892^{* * *}$ & $1.029^{* * *}$ & $1.007^{* * *}$ & $0.508^{* * *}$ & 0.019 \\
\hline & {$[0.010]$} & [0.110] & [0.024] & {$[0.046]$} & {$[0.027]$} \\
\hline ENVSCORE & $\begin{array}{c}-0.734 * * * \\
{[0.246]}\end{array}$ & $\begin{array}{c}200.884^{* * *} \\
{[47.634]}\end{array}$ & $\begin{array}{c}15.784 * * * \\
{[2.639]}\end{array}$ & $\begin{array}{c}-0.893 * * * \\
{[0.222]}\end{array}$ & $\begin{array}{l}-0.019 \\
{[0.165]}\end{array}$ \\
\hline Post_Crisis & $\begin{array}{c}-0.250 * \\
{[0.134]}\end{array}$ & $\begin{array}{c}91.096^{* * *} \\
{[24.769]}\end{array}$ & $\begin{array}{c}6.030 * * * \\
{[1.174]}\end{array}$ & $\begin{array}{c}-0.587^{* * *} \\
{[0.123]}\end{array}$ & $\begin{array}{c}-0.402 * * * \\
{[0.089]}\end{array}$ \\
\hline Post_Crisis*ENVSCORE & $\begin{array}{c}0.609 * * * \\
{[0.219]}\end{array}$ & $\begin{array}{c}-198.174^{* * *} \\
{[47.124]}\end{array}$ & $\begin{array}{c}-13.210 * * * \\
{[2.286]}\end{array}$ & $\begin{array}{c}0.847^{* * *} \\
{[0.216]}\end{array}$ & $\begin{array}{c}0.013 \\
{[0.147]}\end{array}$ \\
\hline \multicolumn{6}{|c|}{ ENVSCORE Equation } \\
\hline $\begin{array}{c}\text { Financial } \\
\text { Performance }\end{array}$ & $0.026^{*}$ & 0.012 & 0.017 & -0.784 & 5.972 \\
\hline & [0.016] & [0.026] & [0.013] & {$[0.494]$} & [3.910] \\
\hline Post_Crisis & $\begin{array}{c}0.310^{* * *} \\
{[0.058]}\end{array}$ & $\begin{array}{c}0.316 \\
{[0.870]}\end{array}$ & $\begin{array}{c}0.309 * * * \\
{[0.065]}\end{array}$ & $\begin{array}{l}-0.293 \\
{[0.351]}\end{array}$ & $\begin{array}{l}2.388 * \\
{[1.374]}\end{array}$ \\
\hline $\begin{array}{l}\text { Post_Crisis*Financial } \\
\text { Performance }\end{array}$ & -0.02 & -0.003 & -0.012 & $0.836^{*}$ & -5.959 \\
\hline & {$[0.016]$} & {$[0.026]$} & {$[0.014]$} & [0.489] & [3.905] \\
\hline Observations & 939 & 624 & 879 & 589 & 1004 \\
\hline \multicolumn{6}{|c|}{ Common Law Countries } \\
\hline & $\begin{array}{l}\text { Net Interest } \\
\text { Margin }\end{array}$ & $\begin{array}{c}\text { Tier1 } \\
\text { Cap_Adq }\end{array}$ & $\begin{array}{l}\text { Non Performing } \\
\text { Loans }\end{array}$ & $\begin{array}{c}\text { Cost Income } \\
\text { Ratio }\end{array}$ & $\begin{array}{l}\text { Excess } \\
\text { Return }\end{array}$ \\
\hline \multicolumn{6}{|c|}{ Financial Performance Equation } \\
\hline $\begin{array}{c}\text { Financial } \\
\text { Performance lag1 }\end{array}$ & $0.968^{* * *}$ & $0.830 * * *$ & $0.921 * * *$ & $0.702 * * *$ & $-0.081^{* * *}$ \\
\hline & [0.012] & [0.078] & [0.012] & {$[0.026]$} & {$[0.027]$} \\
\hline ENVSCORE & $\begin{array}{c}0.998 * * * \\
{[0.160]}\end{array}$ & $\begin{array}{c}84.366^{* * *} \\
{[21.647]}\end{array}$ & $\begin{array}{l}-0.143 \\
{[0.368]}\end{array}$ & $\begin{array}{c}-0.144^{* * *} \\
{[0.042]}\end{array}$ & $\begin{array}{l}0.122 * \\
{[0.073]}\end{array}$ \\
\hline Post_Crisis & $\begin{array}{c}0.266^{* * *} \\
{[0.095]}\end{array}$ & $\begin{array}{c}51.270 * * * \\
{[13.662]}\end{array}$ & $\begin{array}{l}-0.095 \\
{[0.207]}\end{array}$ & $\begin{array}{l}-0.019 \\
{[0.024]}\end{array}$ & $\begin{array}{l}-0.057 \\
{[0.042]}\end{array}$ \\
\hline Post_Crisis*ENVSCORE & $\begin{array}{c}-0.704^{* * *} \\
{[0.136]}\end{array}$ & $\begin{array}{c}-79.880 * * * \\
{[20.668]}\end{array}$ & $\begin{array}{c}0.453 \\
{[0.311]}\end{array}$ & $\begin{array}{c}0.119 * * * \\
{[0.035]}\end{array}$ & $\begin{array}{l}-0.069 \\
{[0.062]}\end{array}$ \\
\hline
\end{tabular}


Table 6. Cont.

\begin{tabular}{|c|c|c|c|c|c|}
\hline \multicolumn{6}{|c|}{ ENVSCORE Equation } \\
\hline $\begin{array}{c}\text { Financial } \\
\text { Performance }\end{array}$ & 0.019 & $0.279 * * *$ & $-0.079 * *$ & $-1.398 * * *$ & 2.956 \\
\hline & [0.015] & {$[0.046]$} & [0.034] & [0.237] & [1.844] \\
\hline Post_Crisis & $\begin{array}{c}0.177 * * * \\
{[0.064]}\end{array}$ & $\begin{array}{c}2.361 * * * \\
{[0.417]}\end{array}$ & $\begin{array}{l}0.090^{* *} \\
{[0.045]}\end{array}$ & $\begin{array}{c}-0.316^{* * *} \\
{[0.092]}\end{array}$ & $\begin{array}{c}0.397^{* * *} \\
{[0.148]}\end{array}$ \\
\hline $\begin{array}{l}\text { Post_Crisis*Financial } \\
\text { Performance }\end{array}$ & -0.004 & $-0.266^{* * *}$ & $0.086^{* *}$ & $1.337^{* * *}$ & -2.941 \\
\hline Observations & $\begin{array}{c}{[0.016]} \\
887\end{array}$ & $\begin{array}{c}{[0.045]} \\
644\end{array}$ & $\begin{array}{c}{[0.034]} \\
912\end{array}$ & $\begin{array}{c}{[0.226]} \\
858\end{array}$ & $\begin{array}{c}{[1.843]} \\
945\end{array}$ \\
\hline \multicolumn{6}{|c|}{ Civil Law Countries } \\
\hline & $\begin{array}{l}\text { Net Interest } \\
\text { Margin }\end{array}$ & $\begin{array}{c}\text { Tier1 } \\
\text { Cap_Adq }\end{array}$ & $\begin{array}{l}\text { Non Performing } \\
\text { Loans }\end{array}$ & $\begin{array}{c}\text { Cost Income } \\
\text { Ratio }\end{array}$ & $\begin{array}{l}\text { Excess } \\
\text { Return }\end{array}$ \\
\hline \multicolumn{6}{|c|}{ Financial Performance Equation } \\
\hline $\begin{array}{c}\text { Financial } \\
\text { Performance_lag1 }\end{array}$ & $0.894^{* * *}$ & $0.864^{* * *}$ & $1.031^{* * *}$ & $0.579 * * *$ & 0.02 \\
\hline SOCSCORE & $\begin{array}{c}{[0.010]} \\
-0.502 * * \\
{[0.226]}\end{array}$ & $\begin{array}{c}{[0.046]} \\
72.424^{* * *} \\
{[22.266]}\end{array}$ & $\begin{array}{c}{[0.022]} \\
12.701 * * * \\
{[1.633]}\end{array}$ & $\begin{array}{c}{[0.064]} \\
-1.700 * * \\
{[0.362]}\end{array}$ & $\begin{array}{l}{[0.027]} \\
-0.011 \\
{[0.152]}\end{array}$ \\
\hline Post_Crisis & $\begin{array}{l}-0.189 \\
{[0.139]}\end{array}$ & $\begin{array}{c}53.984^{* * *} \\
{[18.805]}\end{array}$ & $\begin{array}{c}5.696^{* * *} \\
{[0.959]}\end{array}$ & $\begin{array}{c}-1.291 * * * \\
{[0.254]}\end{array}$ & $\begin{array}{c}-0.413^{* * *} \\
{[0.095]}\end{array}$ \\
\hline Post_Crisis*SOCSCORE & $\begin{array}{l}0.373 * \\
{[0.191]}\end{array}$ & $\begin{array}{l}-70.704 * * * \\
{[21.898]}\end{array}$ & $\begin{array}{c}-9.926^{* * *} \\
{[1.390]}\end{array}$ & $\begin{array}{c}1.598^{* * *} \\
{[0.345]}\end{array}$ & $\begin{array}{c}0.024 \\
{[0.127]}\end{array}$ \\
\hline \multicolumn{6}{|c|}{ SOCSCORE Equation } \\
\hline $\begin{array}{c}\text { Financial } \\
\text { Performance }\end{array}$ & 0.009 & 0.001 & 0.014 & -0.328 & $8.684^{*}$ \\
\hline Post_Crisis & $\begin{array}{l}{[0.014]} \\
0.132 * * \\
{[0.054]}\end{array}$ & $\begin{array}{l}{[0.022]} \\
-0.202 \\
{[0.749]}\end{array}$ & $\begin{array}{c}{[0.012]} \\
0.191 * * * \\
{[0.060]}\end{array}$ & $\begin{array}{l}{[0.410]} \\
-0.199 \\
{[0.288]}\end{array}$ & $\begin{array}{l}{[4.839]} \\
3.180^{*} \\
{[1.698]}\end{array}$ \\
\hline $\begin{array}{l}\text { Post_Crisis*Financial } \\
\text { Performance }\end{array}$ & 0.01 & 0.004 & -0.008 & 0.368 & $-8.647^{*}$ \\
\hline & {$[0.014]$} & [0.022] & {$[0.013]$} & [0.405] & {$[4.830]$} \\
\hline Observations & 939 & 669 & 584 & 589 & 1004 \\
\hline \multicolumn{6}{|c|}{ Common Law Countries } \\
\hline & $\begin{array}{l}\text { Net Interest } \\
\text { Margin }\end{array}$ & $\begin{array}{c}\text { Tier1 } \\
\text { Cap_Adq }\end{array}$ & $\begin{array}{l}\text { Non Performing } \\
\text { Loans }\end{array}$ & $\begin{array}{c}\text { Cost Income } \\
\text { Ratio }\end{array}$ & $\begin{array}{l}\text { Excess } \\
\text { Return }\end{array}$ \\
\hline \multicolumn{6}{|c|}{ Financial Performance Equation } \\
\hline $\begin{array}{c}\text { Financial } \\
\text { Performance_lag1 }\end{array}$ & $0.963^{* * *}$ & $0.803 * * *$ & $0.928^{* * *}$ & $0.702 * * *$ & $-0.079 * * *$ \\
\hline SOCSCORE & $\begin{array}{c}{[0.012]} \\
1.324^{* * *} \\
{[0.223]}\end{array}$ & $\begin{array}{c}{[0.096]} \\
117.542^{* * *} \\
{[36.447]}\end{array}$ & $\begin{array}{l}{[0.012]} \\
-0.456 \\
{[0.480]}\end{array}$ & $\begin{array}{c}{[0.026]} \\
-0.184^{* * *} \\
{[0.057]}\end{array}$ & $\begin{array}{c}{[0.028]} \\
0.105 \\
{[0.098]}\end{array}$ \\
\hline Post_Crisis & $\begin{array}{c}0.499 * * * \\
{[0.122]}\end{array}$ & $\begin{array}{l}64.264^{* * *} \\
{[20.470]}\end{array}$ & $\begin{array}{c}0.048 \\
{[0.264]}\end{array}$ & $\begin{array}{c}-0.056^{*} \\
{[0.031]}\end{array}$ & $\begin{array}{l}-0.048 \\
{[0.053]}\end{array}$ \\
\hline Post_Crisis*SOCSCORE & $\begin{array}{c}-0.982 * * * \\
{[0.191]}\end{array}$ & $\begin{array}{c}-111.216^{* * *} \\
{[34.760]}\end{array}$ & $\begin{array}{c}0.177 \\
{[0.413]}\end{array}$ & $\begin{array}{c}0.160^{* * *} \\
{[0.048]}\end{array}$ & $\begin{array}{l}-0.06 \\
{[0.083]}\end{array}$ \\
\hline \multicolumn{6}{|c|}{ SOCSCORE Equation } \\
\hline $\begin{array}{c}\text { Financial } \\
\text { Performance }\end{array}$ & $0.039 * * *$ & $0.234^{* * *}$ & $-0.129^{* * *}$ & $-1.453 * * *$ & $2.906^{*}$ \\
\hline Post_Crisis & $\begin{array}{l}{[0.014]} \\
0.144^{* *} \\
{[0.059]}\end{array}$ & $\begin{array}{c}{[0.039]} \\
2.006^{* * *} \\
{[0.352]}\end{array}$ & $\begin{array}{l}{[0.031]} \\
-0.038 \\
{[0.041]}\end{array}$ & $\begin{array}{c}{[0.219]} \\
-0.447^{* * *} \\
{[0.085]}\end{array}$ & $\begin{array}{l}{[1.616]} \\
0.300 * * \\
{[0.134]}\end{array}$ \\
\hline $\begin{array}{l}\text { Post_Crisis*Financial } \\
\text { Performance }\end{array}$ & -0.023 & $-0.222 * * *$ & $0.130^{* * *}$ & $1.370^{* * *}$ & $-2.900 *$ \\
\hline & [0.015] & [0.038] & [0.031] & [0.206] & [1.614] \\
\hline Observations & 887 & 644 & 912 & 858 & 945 \\
\hline
\end{tabular}


Table 7. 3SLS estimation for the role of the number of supervisory authorities on banks with comparison between the post and pre-crisis periods in the banking industry. This table reports 3SLS estimations for simultaneous equation system of the relationship between responsibility scores and bank specific financial performance measures for the effect of crisis years with CGVSCORE, ENVSCORE, and SOCSCORE. The definitions of variables are in Appendix A. All regressions control year fixed effects. The sample period is from 2002 to 2015. Robust standard errors presented in brackets are clustered at the firm level, and ${ }^{* * *},{ }^{* *}$, and * denote statistical significance at the $1 \%, 5 \%$, and $10 \%$ levels.

\begin{tabular}{|c|c|c|c|c|c|}
\hline \multicolumn{6}{|c|}{ Countries with Single Bank Supervisory Authorities } \\
\hline & $\begin{array}{l}\text { Net Interest } \\
\text { Margin }\end{array}$ & $\begin{array}{c}\text { Tier1 } \\
\text { Cap_Adq }\end{array}$ & $\begin{array}{c}\text { Non Performing } \\
\text { Loans }\end{array}$ & $\begin{array}{c}\text { Cost Income } \\
\text { Ratio }\end{array}$ & $\begin{array}{l}\text { Excess } \\
\text { Return }\end{array}$ \\
\hline \multicolumn{6}{|c|}{ Financial Performance Equation } \\
\hline \multirow{2}{*}{$\begin{array}{c}\text { Financial } \\
\text { Performance_lag1 }\end{array}$} & $0.920 * * *$ & $0.875^{* * *}$ & $0.999 * * *$ & $0.602 * * *$ & 0.005 \\
\hline & {$[0.008]$} & [0.022] & [0.012] & {$[0.026]$} & [0.022] \\
\hline \multirow[t]{2}{*}{ CGVSCORE } & -0.085 & $34.518^{* * *}$ & $3.257 * * *$ & -0.076 & -0.083 \\
\hline & [0.108] & [5.904] & {$[0.540]$} & [0.073] & [0.067] \\
\hline \multirow[t]{2}{*}{ Post_Crisis } & $-0.165^{* *}$ & $14.770^{* * *}$ & $1.958^{* * *}$ & $-0.144^{* * *}$ & $-0.326^{* * *}$ \\
\hline & {$[0.084]$} & [3.071] & [0.405] & [0.052] & [0.054] \\
\hline \multirow[t]{2}{*}{ Post_Crisis*CGVSCORE } & 0.260 ** & $-33.863^{* * *}$ & $-3.531^{* * *}$ & 0.082 & $0.121 *$ \\
\hline & {$[0.111]$} & [5.875] & [0.555] & {$[0.070]$} & [0.069] \\
\hline \multicolumn{6}{|c|}{ CGVSCORE Equation } \\
\hline \multirow{2}{*}{$\begin{array}{c}\text { Financial } \\
\text { Performance }\end{array}$} & $0.029 * *$ & 0.003 & $0.087^{* * *}$ & $-2.824^{* * *}$ & $11.003^{* * *}$ \\
\hline & [0.011] & {$[0.008]$} & [0.009] & [0.471] & [3.409] \\
\hline \multirow[t]{2}{*}{ Post_Crisis } & $0.216^{* * *}$ & 0.243 & $0.384^{* * *}$ & $-1.402 * * *$ & $2.639 * * *$ \\
\hline & [0.042] & [0.167] & {$[0.041]$} & [0.268] & [0.800] \\
\hline \multirow{2}{*}{$\begin{array}{l}\text { Post_Crisis*Financial } \\
\text { Performance }\end{array}$} & $-0.019 *$ & 0 & $-0.088^{* * *}$ & $2.764^{* * *}$ & $-10.989 * * *$ \\
\hline & {$[0.011]$} & {$[0.008]$} & {$[0.010]$} & {$[0.465]$} & {$[3.412]$} \\
\hline \multirow[t]{3}{*}{ Observations } & 1294 & 892 & 1263 & 915 & 1389 \\
\hline & \multicolumn{4}{|c|}{ Countries with multiple bank supervisory authorities } & \\
\hline & \multirow[t]{2}{*}{$\begin{array}{l}\text { Net Interest } \\
\text { Margin }\end{array}$} & $\begin{array}{c}\text { Tier1 } \\
\text { Cap_Adq }\end{array}$ & $\begin{array}{l}\text { Non Performing } \\
\text { Loans }\end{array}$ & $\begin{array}{c}\text { Cost Income } \\
\text { Ratio }\end{array}$ & $\begin{array}{l}\text { Excess } \\
\text { Return }\end{array}$ \\
\hline \multicolumn{5}{|c|}{ Financial Performance Equation } & \\
\hline \multirow{2}{*}{$\begin{array}{c}\text { Financial } \\
\text { Performance_lag1 }\end{array}$} & $0.960 * * *$ & $0.887^{* * *}$ & $0.830 * * *$ & $0.436^{* * *}$ & $-0.097^{* * *}$ \\
\hline & {$[0.016]$} & {$[0.030]$} & {$[0.024]$} & {$[0.039]$} & [0.036] \\
\hline \multirow[t]{2}{*}{ CGVSCORE } & 0.05 & 10.335 & $2.955^{* * *}$ & $0.297^{* * *}$ & $-0.448^{* * *}$ \\
\hline & {$[0.250]$} & [7.376] & {$[0.604]$} & {$[0.097]$} & [0.111] \\
\hline \multirow[t]{2}{*}{ Post_Crisis } & 0.11 & 8.633 & $2.372^{* * *}$ & $0.163^{* *}$ & $-0.400 * * *$ \\
\hline & [0.189] & {$[6.463]$} & {$[0.441]$} & {$[0.070]$} & [0.084] \\
\hline \multirow[t]{2}{*}{ Post_Crisis*CGVSCORE } & -0.027 & -10.476 & $-3.198 * * *$ & $-0.282 * * *$ & $0.437^{* * *}$ \\
\hline & [0.251] & [7.317] & [0.595] & [0.095] & [0.112] \\
\hline \multicolumn{6}{|c|}{ CGVSCORE Equation } \\
\hline \multirow[t]{2}{*}{$\begin{array}{c}\text { Financial } \\
\text { Performance }\end{array}$} & -0.015 & $0.236^{* * *}$ & -0.03 & $0.948^{* * *}$ & $2.437^{* * *}$ \\
\hline & [0.014] & [0.057] & [0.036] & [0.302] & [0.789] \\
\hline \multirow[t]{2}{*}{ Post_Crisis } & -0.04 & $1.843^{* * *}$ & -0.07 & $0.243^{* *}$ & $0.315^{* *}$ \\
\hline & [0.056] & [0.517] & [0.049] & [0.107] & {$[0.126]$} \\
\hline \multirow{2}{*}{$\begin{array}{l}\text { Post_Crisis*Financial } \\
\text { Performance }\end{array}$} & 0.004 & $-0.227^{* * *}$ & 0.03 & $-0.746^{* * *}$ & $-2.475 * * *$ \\
\hline & [0.014] & [0.056] & [0.035] & [0.278] & [0.798] \\
\hline Observations & 546 & 392 & 545 & 549 & 577 \\
\hline
\end{tabular}


Table 7. Cont.

\begin{tabular}{|c|c|c|c|c|c|}
\hline \multicolumn{6}{|c|}{ Countries with single bank supervisory authorities } \\
\hline & $\begin{array}{l}\text { Net Interest } \\
\text { Margin }\end{array}$ & $\begin{array}{c}\text { Tier1 } \\
\text { Cap_Adq }\end{array}$ & $\begin{array}{c}\text { Non Performing } \\
\text { Loans }\end{array}$ & $\begin{array}{l}\text { Cost Income } \\
\text { Ratio }\end{array}$ & $\begin{array}{l}\text { Excess } \\
\text { Return }\end{array}$ \\
\hline \multicolumn{6}{|c|}{ Financial Performance Equation } \\
\hline \multirow[t]{2}{*}{$\begin{array}{c}\text { Financial } \\
\text { Performance_lag1 }\end{array}$} & $0.920 * * *$ & $1.030^{* * *}$ & $0.992 * * *$ & $0.616^{* * *}$ & 0.008 \\
\hline & [0.008] & [0.067] & {$[0.014]$} & {$[0.030]$} & {$[0.022]$} \\
\hline \multirow[t]{2}{*}{ ENVSCORE } & -0.121 & $130.631^{* * *}$ & $7.339 * * *$ & $-0.447^{* * *}$ & 0.107 \\
\hline & [0.186] & [20.048] & [1.032] & [0.103] & [0.119] \\
\hline \multirow[t]{2}{*}{ Post_Crisis } & -0.134 & $93.093^{* * *}$ & $3.924 * * *$ & $-0.368^{* * *}$ & $-0.218 * * *$ \\
\hline & {$[0.120]$} & [14.997] & {$[0.655]$} & {$[0.074]$} & {$[0.078]$} \\
\hline \multirow{2}{*}{ Post_Crisis*ENVSCORE } & 0.142 & $-128.206^{* * *}$ & $-6.106^{* * *}$ & $0.394^{* * *}$ & -0.088 \\
\hline & [0.165] & [19.765] & [0.923] & [0.095] & [0.106] \\
\hline \multicolumn{6}{|c|}{ ENVSCORE Equation } \\
\hline \multirow[t]{2}{*}{$\begin{array}{c}\text { Financial } \\
\text { Performance }\end{array}$} & $0.045^{* * *}$ & 0.015 & $0.033 * * *$ & $-2.265^{* * *}$ & 43.931 \\
\hline & [0.015] & [0.011] & {$[0.011]$} & {$[0.471]$} & [39.866] \\
\hline \multirow[t]{2}{*}{ Post_Crisis } & $0.293^{* * *}$ & 0.237 & $0.273^{* * *}$ & $-1.069 * * *$ & 9.908 \\
\hline & [0.053] & [0.221] & {$[0.048]$} & {$[0.270]$} & [8.825] \\
\hline \multirow{2}{*}{$\begin{array}{l}\text { Post_Crisis*Financial } \\
\text { Performance }\end{array}$} & $-0.033 * *$ & -0.009 & $-0.028 * *$ & $2.227^{* * *}$ & -43.876 \\
\hline & [0.015] & [0.011] & {$[0.012]$} & [0.467] & [39.824] \\
\hline \multirow[t]{3}{*}{ Observations } & 1294 & 892 & 1263 & 915 & 1389 \\
\hline & \multicolumn{4}{|c|}{ Countries with multiple bank supervisory authorities } & \\
\hline & $\begin{array}{c}\text { Net Interest } \\
\text { Margin }\end{array}$ & $\begin{array}{c}\text { Tier1 } \\
\text { Cap_Adq }\end{array}$ & $\begin{array}{c}\text { Non Performing } \\
\text { Loans }\end{array}$ & $\begin{array}{l}\text { Cost Income } \\
\text { Ratio }\end{array}$ & $\begin{array}{l}\text { Excess } \\
\text { Return }\end{array}$ \\
\hline \multicolumn{6}{|c|}{ Financial Performance Equation } \\
\hline \multirow[t]{2}{*}{$\begin{array}{c}\text { Financial } \\
\text { Performance_lag1 }\end{array}$} & $1.037^{* * *}$ & $0.625^{* * *}$ & $0.816^{* * *}$ & $0.595^{* * *}$ & $-0.124 * *$ \\
\hline & {$[0.036]$} & [0.203] & {$[0.027]$} & {$[0.091]$} & {$[0.060]$} \\
\hline \multirow[t]{2}{*}{ ENVSCORE } & $5.535 * * *$ & $141.312 * *$ & $-3.560 * *$ & $-1.018^{* *}$ & $1.864^{* * *}$ \\
\hline & [1.125] & [59.030] & [1.546] & [0.505] & [0.654] \\
\hline \multirow[t]{2}{*}{ Post_Crisis } & $0.671^{* * *}$ & $45.590 * *$ & -0.243 & $-0.106^{*}$ & 0.052 \\
\hline & {$[0.240]$} & {$[21.464]$} & {$[0.248]$} & {$[0.063]$} & [0.092] \\
\hline \multirow[t]{2}{*}{ Post_Crisis*ENVSCORE } & $-3.907^{* * *}$ & $-124.851^{* *}$ & $2.731 * *$ & $0.731 *$ & $-1.283 * * *$ \\
\hline & [0.806] & [52.640] & [1.107] & {$[0.377]$} & [0.459] \\
\hline \multicolumn{6}{|c|}{ ENVSCORE Equation } \\
\hline \multirow{2}{*}{$\begin{array}{c}\text { Financial } \\
\text { Performance }\end{array}$} & 0.012 & $0.426^{* * *}$ & $-0.222 * * *$ & $-1.343^{* * *}$ & 36.834 \\
\hline & [0.017] & [0.089] & [0.035] & {$[0.296]$} & {$[67.868]$} \\
\hline \multirow[t]{2}{*}{ Post_Crisis } & $0.262 * * *$ & $3.795^{* * *}$ & -0.056 & $-0.318^{* * *}$ & 5.49 \\
\hline & {$[0.068]$} & {$[0.831]$} & {$[0.055]$} & [0.113] & [9.797] \\
\hline \multirow{2}{*}{$\begin{array}{l}\text { Post_Crisis*Financial } \\
\text { Performance }\end{array}$} & -0.024 & $-0.400^{* * *}$ & $0.223 * * *$ & $1.337^{* * *}$ & -37.129 \\
\hline & {$[0.017]$} & [0.086] & {$[0.034]$} & {$[0.273]$} & {$[68.471]$} \\
\hline \multirow[t]{3}{*}{ Observations } & 546 & 392 & 545 & 549 & 577 \\
\hline & Countries & ith single ba & supervisory autho & & \\
\hline & $\begin{array}{l}\text { Net Interest } \\
\text { Margin }\end{array}$ & $\begin{array}{c}\text { Tier1 } \\
\text { Cap_Adq }\end{array}$ & $\begin{array}{c}\text { Non Performing } \\
\text { Loans }\end{array}$ & $\begin{array}{l}\text { Cost Income } \\
\text { Ratio }\end{array}$ & $\begin{array}{l}\text { Excess } \\
\text { Return }\end{array}$ \\
\hline & & nancial Perfor & ince Equation & & \\
\hline $\begin{array}{c}\text { Financial } \\
\text { Performance lag1 }\end{array}$ & $0.918^{* * *}$ & $0.849 * * *$ & $1.015^{* * *}$ & $0.662 * * *$ & 0.009 \\
\hline & {$[0.008]$} & [0.031] & [0.015] & [0.038] & {$[0.022]$} \\
\hline SOCSCORE & -0.028 & $53.086^{* * *}$ & $7.654 * * *$ & $-0.997 * * *$ & 0.047 \\
\hline & [0.167] & [13.120] & [0.847] & [0.151] & [0.102] \\
\hline Post_Crisis & -0.09 & $42.175^{* * *}$ & $4.776^{* * *}$ & $-0.795^{* * *}$ & $-0.257^{* * *}$ \\
\hline & {$[0.123]$} & [10.907] & {$[0.641]$} & [0.119] & [0.078] \\
\hline Post_Crisis*SOCSCORE & 0.061 & $-51.896^{* * *}$ & $-6.647^{* * *}$ & $0.891^{* * *}$ & -0.019 \\
\hline & [0.149] & [12.932] & [0.784] & [0.141] & {$[0.092]$} \\
\hline
\end{tabular}


Table 7. Cont.

\begin{tabular}{|c|c|c|c|c|c|}
\hline \multicolumn{6}{|c|}{ SOCSCORE Equation } \\
\hline Financial & $0.026^{*}$ & 0.009 & $0.024^{* *}$ & $-1.171^{* * *}$ & $25.470 *$ \\
\hline & {$[0.014]$} & [0.010] & {$[0.011]$} & [0.385] & [14.188] \\
\hline Post_Crisis & $\begin{array}{c}0.148^{* * *} \\
{[0.049]}\end{array}$ & $\begin{array}{c}0.111 \\
{[0.198]}\end{array}$ & $\begin{array}{c}0.191 * * * \\
{[0.045]}\end{array}$ & $\begin{array}{c}-0.511^{* *} \\
{[0.222]}\end{array}$ & $\begin{array}{l}5.846^{*} \\
{[3.217]}\end{array}$ \\
\hline $\begin{array}{l}\text { Post_Crisis*Financial } \\
\text { Performance }\end{array}$ & -0.003 & -0.004 & $-0.020 *$ & $1.039 * * *$ & $-25.434 *$ \\
\hline & [0.014] & [0.010] & {$[0.011]$} & [0.382] & [14.177] \\
\hline Observations & 1294 & 892 & 1263 & 915 & 1389 \\
\hline \multicolumn{6}{|c|}{ Countries with multiple bank supervisory authorities } \\
\hline & $\begin{array}{l}\text { Net Interest } \\
\text { Margin }\end{array}$ & $\begin{array}{c}\text { Tier1 } \\
\text { Cap_Adq }\end{array}$ & $\begin{array}{c}\text { Non Performing } \\
\text { Loans }\end{array}$ & $\begin{array}{c}\text { Cost Income } \\
\text { Ratio }\end{array}$ & $\begin{array}{l}\text { Excess } \\
\text { Return }\end{array}$ \\
\hline \multicolumn{6}{|c|}{ Financial Performance Equation } \\
\hline $\begin{array}{c}\text { Financial } \\
\text { Performance_lag1 }\end{array}$ & $1.019 * * *$ & $0.546^{*}$ & $0.796^{* * *}$ & $0.502 * * *$ & -0.052 \\
\hline & {$[0.033]$} & {$[0.285]$} & [0.051] & [0.054] & {$[0.046]$} \\
\hline SOCSCORE & $\begin{array}{c}4.898^{* * *} \\
{[1.092]}\end{array}$ & $\begin{array}{c}182.146 \\
{[111.821]}\end{array}$ & $\begin{array}{c}-10.864^{* * *} \\
{[2.612]}\end{array}$ & $\begin{array}{l}-0.578 \\
{[0.363]}\end{array}$ & $\begin{array}{l}0.938^{* *} \\
{[0.427]}\end{array}$ \\
\hline Post_Crisis & $\begin{array}{c}1.221^{* * *} \\
{[0.322]}\end{array}$ & $\begin{array}{c}46.878 \\
{[31.299]}\end{array}$ & $\begin{array}{c}-2.109^{* * *} \\
{[0.705]}\end{array}$ & $\begin{array}{l}-0.153 \\
{[0.097]}\end{array}$ & $\begin{array}{c}0.1 \\
{[0.112]}\end{array}$ \\
\hline Post_Crisis*SOCSCORE & $\begin{array}{c}-3.272^{* * *} \\
{[0.756]}\end{array}$ & $\begin{array}{c}-160.523 \\
{[99.460]}\end{array}$ & $\begin{array}{c}7.141^{* * *} \\
{[1.830]}\end{array}$ & $\begin{array}{c}0.371 \\
{[0.264]}\end{array}$ & $\begin{array}{c}-0.603 * * \\
{[0.289]}\end{array}$ \\
\hline \multicolumn{6}{|c|}{ SOCSCORE Equation } \\
\hline $\begin{array}{c}\text { Financial } \\
\text { Performance }\end{array}$ & 0.01 & $0.361 * * *$ & $-0.131^{* * *}$ & $-1.174 * * *$ & 3.916 \\
\hline Post_Crisis & $\begin{array}{c}{[0.014]} \\
0.089 \\
{[0.060]}\end{array}$ & $\begin{array}{c}{[0.073]} \\
3.210^{* * * *} \\
{[0.681]}\end{array}$ & $\begin{array}{c}{[0.029]} \\
-0.080 * \\
{[0.047]}\end{array}$ & $\begin{array}{c}{[0.241]} \\
-0.393^{* * *} \\
{[0.090]}\end{array}$ & $\begin{array}{c}{[3.167]} \\
0.579 \\
{[0.445]}\end{array}$ \\
\hline $\begin{array}{l}\text { Post_Crisis*Financial } \\
\text { Performance }\end{array}$ & -0.018 & $-0.337^{* * *}$ & $0.123 * * *$ & $1.133^{* * *}$ & -3.9 \\
\hline & [0.014] & [0.071] & [0.029] & [0.227] & [3.190] \\
\hline Observations & 546 & 392 & 545 & 549 & 577 \\
\hline
\end{tabular}

Table 8. 3SLS estimation for the role of provider of banking supervision with comparison between the post and pre-crisis periods in the banking industry. This table reports 3SLS estimations for simultaneous equation system of the relationship between responsibility scores and bank specific financial performance measures for the effect of crisis years with CGVSCORE, ENVSCORE, and SOCSCORE. The definitions of variables are in Appendix A. All regressions control year fixed effects. The sample period is from 2002 to 2015. Robust standard errors presented in brackets are clustered at the firm level, and ${ }^{* * *},{ }^{* *}$, and * denote statistical significance at the $1 \%, 5 \%$, and $10 \%$ levels.

\begin{tabular}{cccccc}
\hline \multicolumn{5}{c}{ Countries with Banking Supervision Conducted by Agencies Outside of the Central Bank } \\
\hline & $\begin{array}{c}\text { Net Interest } \\
\text { Margin }\end{array}$ & $\begin{array}{c}\text { Tier1 } \\
\text { Cap_Adq }\end{array}$ & $\begin{array}{c}\text { Non Performing } \\
\text { Loans }\end{array}$ & $\begin{array}{c}\text { Cost Income } \\
\text { Ratio }\end{array}$ & $\begin{array}{c}\text { Excess } \\
\text { Return }\end{array}$ \\
\hline & & Financial Performance Equation & & \\
Financial & $0.939^{* * *}$ & $0.960^{* * *}$ & $0.909^{* * *}$ & $0.461^{* * *}$ & -0.017 \\
Performance_lag1 & {$[0.011]$} & {$[0.036]$} & {$[0.016]$} & {$[0.037]$} & {$[0.031]$} \\
& $-0.161^{*}$ & $-49.606^{* * *}$ & $1.735^{* * *}$ & 0.052 & $-0.128^{*}$ \\
CGVSCORE & {$[0.086]$} & {$[9.280]$} & {$[0.290]$} & {$[0.090]$} & {$[0.068]$} \\
Post_Crisis & $-0.181^{* *}$ & $-31.736^{* * *}$ & $1.231^{* * *}$ & $-0.122^{*}$ & $-0.386^{* * *}$ \\
& {$[0.083]$} & {$[6.541]$} & {$[0.261]$} & {$[0.068]$} & {$[0.064]$} \\
Post_Crisis*CGVSCORE & $0.263^{* * *}$ & $49.653^{* * *}$ & $-1.671^{* * *}$ & 0.002 & $0.166^{* *}$ \\
& {$[0.092]$} & {$[9.291]$} & {$[0.300]$} & {$[0.092]$} & {$[0.073]$} \\
\hline
\end{tabular}


Table 8. Cont.

\begin{tabular}{|c|c|c|c|c|c|}
\hline \multicolumn{6}{|c|}{ CGVSCORE Equation } \\
\hline \multirow{2}{*}{$\begin{array}{c}\text { Financial } \\
\text { Performance }\end{array}$} & 0.016 & $-0.036^{* * *}$ & $0.030^{* * *}$ & 0.483 & $4.393^{* * *}$ \\
\hline & [0.017] & [0.013] & [0.008] & {$[0.350]$} & {$[1.314]$} \\
\hline \multirow{2}{*}{ Post_Crisis } & $0.168^{* * *}$ & -0.152 & $0.225^{* * *}$ & 0.355 & $1.330 * * *$ \\
\hline & {$[0.051]$} & [0.152] & {$[0.042]$} & {$[0.216]$} & {$[0.378]$} \\
\hline \multirow[t]{2}{*}{$\begin{array}{c}\text { Post_Crisis*Financial } \\
\text { Performance }\end{array}$} & -0.011 & $0.039 * * *$ & $-0.028^{* * *}$ & -0.389 & $-4.361^{* * *}$ \\
\hline & {$[0.017]$} & [0.013] & [0.008] & {$[0.350]$} & [1.317] \\
\hline \multirow[t]{3}{*}{ Observations } & 710 & 459 & 660 & 507 & 769 \\
\hline & \multicolumn{5}{|c|}{ ountries with banking supervision conducted by the central bank } \\
\hline & $\begin{array}{l}\text { Net Interest } \\
\text { Margin }\end{array}$ & $\begin{array}{c}\text { Tier1 } \\
\text { Cap_Adq }\end{array}$ & $\begin{array}{c}\text { Non Performing } \\
\text { Loans }\end{array}$ & $\begin{array}{l}\text { Cost Income } \\
\text { Ratio }\end{array}$ & $\begin{array}{l}\text { Excess } \\
\text { Return }\end{array}$ \\
\hline \multicolumn{6}{|c|}{ Financial Performance Equation } \\
\hline \multirow[t]{2}{*}{$\begin{array}{c}\text { Financial } \\
\text { Performance_lag1 }\end{array}$} & $0.935^{* * *}$ & $1.051^{* * *}$ & $0.971^{* * *}$ & $0.627^{* * *}$ & -0.035 \\
\hline & [0.009] & [0.056] & [0.018] & [0.028] & [0.023] \\
\hline CGVSCORE & $\begin{array}{c}-0.553^{* *} \\
{[0.259]}\end{array}$ & $\begin{array}{c}104.167^{* * *} \\
{[12.547]}\end{array}$ & $\begin{array}{c}12.286^{* * *} \\
{[1.505]}\end{array}$ & $\begin{array}{c}0.518^{* * *} \\
{[0.097]}\end{array}$ & $\begin{array}{l}-0.129 \\
{[0.127]}\end{array}$ \\
\hline Post_Crisis & $\begin{array}{c}-0.302 * * \\
{[0.143]}\end{array}$ & $\begin{array}{c}59.073 * * * \\
{[7.970]}\end{array}$ & $\begin{array}{c}6.715^{* * *} \\
{[0.845]}\end{array}$ & $\begin{array}{c}0.346^{* * *} \\
{[0.063]}\end{array}$ & $\begin{array}{c}-0.236 * * * \\
{[0.074]}\end{array}$ \\
\hline Post_Crisis*CGVSCORE & $\begin{array}{c}0.638^{* * *} \\
{[0.226]}\end{array}$ & $\begin{array}{c}-101.037^{* * *} \\
{[12.274]}\end{array}$ & $\begin{array}{c}-11.577^{* * *} \\
{[1.333]}\end{array}$ & $\begin{array}{c}-0.492 * * * \\
{[0.087]}\end{array}$ & $\begin{array}{l}0.187^{*} \\
{[0.111]}\end{array}$ \\
\hline \multicolumn{6}{|c|}{ CGVSCORE Equation } \\
\hline \multirow[t]{2}{*}{$\begin{array}{c}\text { Financial } \\
\text { Performance }\end{array}$} & $0.037^{* * *}$ & $0.060 * * *$ & $0.094^{* * *}$ & 0.239 & $9.536^{* * *}$ \\
\hline & {$[0.011]$} & [0.012] & {$[0.018]$} & [0.413] & {$[2.666]$} \\
\hline Post_Crisis & $\begin{array}{c}0.144^{* * *} \\
{[0.048]}\end{array}$ & $\begin{array}{c}0.936^{* * *} \\
{[0.223]}\end{array}$ & $\begin{array}{c}0.134^{* * *} \\
{[0.039]}\end{array}$ & $\begin{array}{c}0.019 \\
{[0.136]}\end{array}$ & $\begin{array}{c}1.218^{* * *} \\
{[0.371]}\end{array}$ \\
\hline \multirow[t]{2}{*}{$\begin{array}{l}\text { Post_Crisis*Financial } \\
\text { Performance }\end{array}$} & $-0.031^{* * *}$ & $-0.054^{* * *}$ & $-0.092 * * *$ & -0.153 & $-9.532 * * *$ \\
\hline & [0.012] & [0.012] & [0.018] & [0.410] & {$[2.666]$} \\
\hline Observations & 1130 & 825 & 1148 & 957 & 1197 \\
\hline \multicolumn{6}{|c|}{ Countries with banking supervision conducted by agencies outside of the central bank } \\
\hline & $\begin{array}{c}\text { Net Interest } \\
\text { Margin }\end{array}$ & $\begin{array}{c}\text { Tier1 } \\
\text { Cap_Adq }\end{array}$ & $\begin{array}{c}\text { Non Performing } \\
\text { Loans }\end{array}$ & $\begin{array}{l}\text { Cost Income } \\
\text { Ratio }\end{array}$ & $\begin{array}{l}\text { Excess } \\
\text { Return }\end{array}$ \\
\hline \multicolumn{6}{|c|}{ Financial Performance Equation } \\
\hline \multirow[t]{2}{*}{$\begin{array}{c}\text { Financial } \\
\text { Performance_lag1 }\end{array}$} & $0.933^{* * *}$ & $1.034^{* * *}$ & $0.942^{* * *}$ & $0.469^{* * *}$ & -0.01 \\
\hline & {$[0.011]$} & [0.100] & {$[0.023]$} & [0.043] & [0.031] \\
\hline ENVSCORE & $\begin{array}{c}0.095 \\
{[0.163]}\end{array}$ & $\begin{array}{c}147.225^{* * *} \\
{[35.691]}\end{array}$ & $\begin{array}{c}5.913^{* * *} \\
{[0.757]}\end{array}$ & $\begin{array}{c}-0.549 * * * \\
{[0.161]}\end{array}$ & $\begin{array}{c}0.001 \\
{[0.137]}\end{array}$ \\
\hline Post_Crisis & $\begin{array}{c}0.012 \\
{[0.110]}\end{array}$ & $\begin{array}{c}125.616^{* * *} \\
{[30.650]}\end{array}$ & $\begin{array}{c}3.400 * * * \\
{[0.505]}\end{array}$ & $\begin{array}{c}-0.454^{* * *} \\
{[0.107]}\end{array}$ & $\begin{array}{c}-0.313^{* * *} \\
{[0.090]}\end{array}$ \\
\hline Post_Crisis*ENVSCORE & $\begin{array}{l}-0.119 \\
{[0.139]}\end{array}$ & $\begin{array}{c}-144.680^{* * *} \\
{[35.205]}\end{array}$ & $\begin{array}{c}-4.857^{* * *} \\
{[0.648]}\end{array}$ & $\begin{array}{c}0.487^{* * *} \\
{[0.145]}\end{array}$ & $\begin{array}{c}0.005 \\
{[0.119]}\end{array}$ \\
\hline \multicolumn{6}{|c|}{ ENVSCORE Equation } \\
\hline \multirow[t]{2}{*}{$\begin{array}{c}\text { Financial } \\
\text { Performance }\end{array}$} & 0.032 & 0.005 & -0.003 & 0.568 & $9.038^{* *}$ \\
\hline & [0.024] & [0.019] & [0.011] & [0.458] & [4.550] \\
\hline Post_Crisis & $\begin{array}{c}0.216^{* * *} \\
{[0.072]}\end{array}$ & $\begin{array}{l}-0.069 \\
{[0.219]}\end{array}$ & $\begin{array}{c}0.156^{* * *} \\
{[0.059]}\end{array}$ & $\begin{array}{c}0.445 \\
{[0.278]}\end{array}$ & $\begin{array}{l}2.587^{* *} \\
{[1.231]}\end{array}$ \\
\hline \multirow{2}{*}{$\begin{array}{l}\text { Post_Crisis*Financial } \\
\text { Performance }\end{array}$} & -0.016 & 0.005 & 0.013 & -0.481 & $-9.006^{* *}$ \\
\hline & {$[0.024]$} & [0.019] & {$[0.011]$} & {$[0.453]$} & {$[4.545]$} \\
\hline Observations & 710 & 459 & 660 & 507 & 769 \\
\hline
\end{tabular}


Table 8. Cont.

\begin{tabular}{|c|c|c|c|c|c|}
\hline \multicolumn{6}{|c|}{ Countries with banking supervision conducted by the central bank } \\
\hline & $\begin{array}{l}\text { Net Interest } \\
\text { Margin }\end{array}$ & $\begin{array}{c}\text { Tier1 } \\
\text { Cap_Adq }\end{array}$ & $\begin{array}{l}\text { Non Performing } \\
\text { Loans }\end{array}$ & $\begin{array}{c}\text { Cost Income } \\
\text { Ratio }\end{array}$ & $\begin{array}{l}\text { Excess } \\
\text { Return }\end{array}$ \\
\hline \multicolumn{6}{|c|}{ Financial Performance Equation } \\
\hline \multirow{2}{*}{$\begin{array}{c}\text { Financial } \\
\text { Performance_lag1 }\end{array}$} & $0.930 * * *$ & $0.816^{* * *}$ & $0.974^{* * *}$ & $0.674^{* * *}$ & -0.031 \\
\hline & [0.009] & [0.076] & [0.015] & [0.030] & [0.024] \\
\hline ENVSCORE & $\begin{array}{c}0.832 * * * \\
{[0.237]}\end{array}$ & $\begin{array}{c}122.434^{* * *} \\
{[36.850]}\end{array}$ & $\begin{array}{c}-5.351 * * * \\
{[1.234]}\end{array}$ & $\begin{array}{c}-0.550 * * * \\
{[0.097]}\end{array}$ & $\begin{array}{l}0.244^{* *} \\
{[0.116]}\end{array}$ \\
\hline Post_Crisis & $\begin{array}{l}0.250^{* *} \\
{[0.108]}\end{array}$ & $\begin{array}{c}50.955^{* * *} \\
{[16.645]}\end{array}$ & $\begin{array}{c}-1.322 * * * \\
{[0.457]}\end{array}$ & $\begin{array}{c}-0.103^{* * *} \\
{[0.037]}\end{array}$ & $\begin{array}{l}-0.061 \\
{[0.050]}\end{array}$ \\
\hline Post_Crisis*ENVSCORE & $\begin{array}{l}-0.677^{* * *} \\
{[0.207]}\end{array}$ & $\begin{array}{c}-118.585^{* * *} \\
{[35.895]}\end{array}$ & $\begin{array}{c}5.038^{* * *} \\
{[1.064]}\end{array}$ & $\begin{array}{c}0.502 * * * \\
{[0.088]}\end{array}$ & $\begin{array}{c}-0.213^{* *} \\
{[0.100]}\end{array}$ \\
\hline \multicolumn{6}{|c|}{ ENVSCORE Equation } \\
\hline \multirow{2}{*}{$\begin{array}{c}\text { Financial } \\
\text { Performance }\end{array}$} & $0.031^{* *}$ & $0.085^{* * *}$ & $-0.097^{* * *}$ & $-1.311^{* *}$ & $-2.794^{* * *}$ \\
\hline & [0.013] & {$[0.014]$} & {$[0.018]$} & {$[0.523]$} & [0.891] \\
\hline Post_Crisis & $\begin{array}{c}0.273^{* * *} \\
{[0.056]}\end{array}$ & $\begin{array}{c}1.469^{* * *} \\
{[0.270]}\end{array}$ & $\begin{array}{l}0.076 * \\
{[0.043]}\end{array}$ & $\begin{array}{c}-0.286 * \\
{[0.173]}\end{array}$ & $\begin{array}{l}-0.164 \\
{[0.132]}\end{array}$ \\
\hline \multirow{2}{*}{$\begin{array}{l}\text { Post_Crisis*Financial } \\
\text { Performance }\end{array}$} & $-0.025 *$ & $-0.075^{* * *}$ & $0.101^{* * *}$ & $1.308^{* *}$ & $2.789^{* * *}$ \\
\hline & {$[0.014]$} & [0.014] & [0.018] & [0.523] & [0.894] \\
\hline Observations & 1130 & 825 & 1148 & 957 & 1197 \\
\hline \multicolumn{6}{|c|}{ Countries with banking supervision conducted by agencies outside of the central bank } \\
\hline & $\begin{array}{l}\text { Net Interest } \\
\text { Margin }\end{array}$ & $\begin{array}{c}\text { Tier1 } \\
\text { Cap_Adq }\end{array}$ & $\begin{array}{l}\text { Non Performing } \\
\text { Loans }\end{array}$ & $\begin{array}{l}\text { Cost Income } \\
\text { Ratio }\end{array}$ & $\begin{array}{l}\text { Excess } \\
\text { Return }\end{array}$ \\
\hline \multicolumn{6}{|c|}{ Financial Performance Equation } \\
\hline \multirow[t]{2}{*}{$\begin{array}{c}\text { Financial } \\
\text { Performance_lag1 }\end{array}$} & $0.934^{* * *}$ & $0.966^{* * *}$ & $0.962 * * *$ & $0.508^{* * *}$ & -0.014 \\
\hline & {$[0.011]$} & [0.071] & [0.021] & [0.046] & [0.031] \\
\hline \multirow[t]{2}{*}{ SOCSCORE } & -0.033 & $102.941^{* * *}$ & $4.421^{* * *}$ & $-0.845^{* * *}$ & -0.062 \\
\hline & [0.141] & [26.383] & {$[0.471]$} & [0.186] & [0.108] \\
\hline \multirow[t]{2}{*}{ Post_Crisis } & -0.077 & $85.424^{* * *}$ & $2.981 * * *$ & $-0.720 * * *$ & $-0.382 * * *$ \\
\hline & [0.109] & [21.976] & [0.394] & [0.144] & [0.084] \\
\hline \multirow[t]{2}{*}{ Post_Crisis*SOCSCORE } & 0.02 & $-101.107^{* * *}$ & $-3.569 * * *$ & $0.735 * * *$ & 0.105 \\
\hline & {$[0.118]$} & {$[26.055]$} & [0.418] & {$[0.171]$} & [0.093] \\
\hline \multicolumn{6}{|c|}{ SOCSCORE Equation } \\
\hline \multirow[t]{2}{*}{$\begin{array}{c}\text { Financial } \\
\text { Performance }\end{array}$} & 0.018 & -0.01 & $-0.021 * *$ & 0.317 & $5.950 * * *$ \\
\hline & [0.021] & [0.016] & [0.009] & [0.363] & [2.287] \\
\hline \multirow[t]{2}{*}{ Post_Crisis } & 0.081 & -0.049 & 0.032 & 0.209 & $1.763^{* * *}$ \\
\hline & [0.064] & [0.188] & [0.053] & {$[0.221]$} & [0.655] \\
\hline \multirow[t]{2}{*}{$\begin{array}{c}\text { Post_Crisis*Financial } \\
\text { Performance }\end{array}$} & 0.005 & 0.014 & $0.030 * * *$ & -0.286 & $-5.856^{* *}$ \\
\hline & [0.021] & [0.016] & {$[0.010]$} & [0.359] & {$[2.280]$} \\
\hline Observations & 710 & 459 & 660 & 507 & 769 \\
\hline \multicolumn{6}{|c|}{ Countries with banking supervision conducted by the central bank } \\
\hline & $\begin{array}{l}\text { Net Interest } \\
\text { Margin }\end{array}$ & $\begin{array}{c}\text { Tier1 } \\
\text { Cap_Adq }\end{array}$ & $\begin{array}{l}\text { Non Performing } \\
\text { Loans }\end{array}$ & $\begin{array}{l}\text { Cost Income } \\
\text { Ratio }\end{array}$ & $\begin{array}{l}\text { Excess } \\
\text { Return }\end{array}$ \\
\hline & & inancial Perfor & Ince Equation & & \\
\hline $\begin{array}{c}\text { Financial } \\
\text { Performance_lag1 }\end{array}$ & $0.926^{* * *}$ & $1.076^{* * *}$ & $0.978^{* * *}$ & $0.672 * * *$ & -0.023 \\
\hline & {$[0.009]$} & {$[0.084]$} & {$[0.014]$} & [0.029] & {$[0.025]$} \\
\hline SOCSCORE & $1.184^{* * *}$ & $-118.673^{* * *}$ & $-4.433^{* * *}$ & $-0.487^{* * *}$ & $0.303 *$ \\
\hline & {$[0.320]$} & [25.546] & [1.473] & [0.149] & [0.164] \\
\hline Post_Crisis & $\begin{array}{l}0.481^{* * *} \\
{[0.152]}\end{array}$ & $\begin{array}{c}-61.492 * * * \\
{[13.344]}\end{array}$ & $\begin{array}{c}-1.484^{* *} \\
{[0.630]}\end{array}$ & $\begin{array}{l}-0.159 * * * \\
{[0.060]}\end{array}$ & $\begin{array}{l}-0.005 \\
{[0.075]}\end{array}$ \\
\hline Post_Crisis*SOCSCORE & $\begin{array}{c}-0.986^{* * *} \\
{[0.277]}\end{array}$ & $\begin{array}{c}116.831^{* * *} \\
{[24.999]}\end{array}$ & $\begin{array}{l}4.017^{* * *} \\
{[1.279]}\end{array}$ & $\begin{array}{c}0.464^{* * *} \\
{[0.135]}\end{array}$ & $\begin{array}{l}-0.269^{*} \\
{[0.141]}\end{array}$ \\
\hline & & SOCSCOR & quation & & \\
\hline $\begin{array}{c}\text { Financial } \\
\text { Performance }\end{array}$ & $0.046^{* * *}$ & $0.084^{* * *}$ & $-0.114^{* * *}$ & $-1.308^{* * *}$ & $-3.233^{* * *}$ \\
\hline & [0.012] & {$[0.012]$} & [0.017] & [0.493] & [0.912] \\
\hline Post_Crisis & $\begin{array}{c}0.186^{* * *} \\
{[0.052]}\end{array}$ & $\begin{array}{c}1.360 * * * \\
{[0.235]}\end{array}$ & $\begin{array}{l}-0.048 \\
{[0.040]}\end{array}$ & $\begin{array}{c}-0.398^{* *} \\
{[0.163]}\end{array}$ & $\begin{array}{c}-0.350 * * \\
{[0.137]}\end{array}$ \\
\hline $\begin{array}{c}\text { Post_Crisis*Financial } \\
\text { Performance }\end{array}$ & $-0.030^{* *}$ & $-0.072^{* * *}$ & $0.118^{* * *}$ & $1.348^{* * *}$ & $3.210^{* * *}$ \\
\hline & [0.013] & [0.012] & [0.017] & [0.493] & [0.913] \\
\hline Observations & 1130 & 825 & 1148 & 957 & 1197 \\
\hline
\end{tabular}




\section{Conclusions}

Banks are special: they are intermediaries and both sides of their balance sheet consist of financial assets that are used as money. As a result, they are highly regulated. We study if, how and why banks' financial performance relates to their responsibility. We zoom in on the finance-responsibility nexus based on detailed bank-specific financial performance indicators used by their regulatory and supervisory authorities. Relying on a large international sample for the 2002-2015 period, we model the financial performance-responsibility nexus and estimate this with the help of a wide array of indicators.

We find that in several cases there is a significant association between bank performance and responsibility. In particular, banks' capital adequacy is driving their responsibility and, therefore, responsibility signals bank health. As such, responsibility has an important reputational effect. We also establish that financial performance most of the time positively affects banks' responsibility, and not the other way around. This implies that especially the available funds hypothesis seems to hold (see $[49,50])$. This hypothesis argues that investment in responsibility is costly and that better performing firms will make more expenses in environmentally and socially advantageous activities. After the financial crisis, the finance-responsibility nexus did significantly change and the interaction between the two types of performance weakened. We find that the institutional setting of the banks can play an important role as we detect differences in the finance-responsibility nexus-and in relation to the global financial crisis-between banks operating in civil versus common law countries and between those supervised by a single bank authority and those supervised by multiple agencies.

Our findings support those of $[2,8,45]$ about the specific position of banks compared to other industries in relation to responsibility. The results of our study align with those in [15] regarding the increase in financial performance after the crisis in the US and show this holds in other jurisdictions as well. Further, we complement the findings in [24], where the financial industry was not included in the analysis about the finance-responsibility performance nexus in relation to the global financial crisis, as well as those of [13], who did not explicitly account for responsibility. We also extend the analysis of $[23,24]$ regarding the role of the legal system in the finance-responsibility nexus. Last is that we extend the research of [18] regarding the role of bank supervision. Further research is required to develop a grounding framework for why and how institutions and development exactly influence the nexus in relation to the global financial crisis.

Our study contributes to the literature on the responsibility of banks by systematically investigating competing hypotheses for a large and international sample of firms. We rely on detailed bank specific performance measures where other studies use more general performance measures, because bank specific measures are more relevant as the economic function of banks is very different from that of other types of firms. At the same time, this is a limitation of our study as it is very hard to compare financial performance of banks with that of firms in other industries due to the specific operational and regulatory features of the banking industry. Therefore, the inter-industry comparison can only be undertaken in a generic manner. Another limitation rests in the quality of the data about CSR. We use fine-grained indicators of responsibility, as advocated in [31], and show that this increases our understanding of the finance-responsibility nexus. However, we need to point out that responsibility cannot be directly observed and we use proxies, which might result in measurement error and underestimation [101]. As long as there are no generally accepted standards regarding their reporting and without independent external validation and auditing of such reports, we think it is hard to substantiate claims by rating organizations about firms' responsibility and the results from studies using these data need to be handled with great care. We look forward to more direct, reliable and validated metrics regarding responsibility. We use a three stages equation system, which takes advantage of the correlation in error terms to arrive at estimates that are more efficient. There is no perfect estimation method to deal with endogeneity. The potential for a simultaneous effect between financial performance and responsibility scores is a limitation of our study. Future research could examine the cause-effect relationship between both variables, for example, by using lags in the specification of the models. 
Author Contributions: Both authors equally contributed to this manuscript.

Funding: This research received no external funding.

Acknowledgments: We very much appreciate the thoughtful comments and suggestions of four anonymous reviewers.

Conflicts of Interest: The authors declare no conflict of interest. This research did not receive any specific grant from funding agencies in the public, commercial or not-for-profit sectors.

\section{Appendix A Definitions of variables.}

Table A1. This table provides definitions of all variables used in analyses.

\begin{tabular}{cl}
\hline Variables & \multicolumn{1}{c}{ Definitions } \\
\hline & Firm-level corporate governance scor \\
& with following components: \\
CGVSCORE & (1) CGBF Board Functions \\
(Corporate Governance Score) & (2) CGBS Board Structure \\
& (3) CGCP Compensation Policy \\
& (4) CGVS Vision and Strategy \\
& (5) CGSR Shareholder Rights
\end{tabular}

Firm-level environmental scores from ASSET4 with following components:

ENVSCORE

(Environmental Score)
(1) ENER Emission Reduction

(2) ENPI Product Innovation

(3) ENRR Resource Reduction

Firm-level social scores from ASSET4 with following components:

(1) SOPR Product Responsibility

(2) SOCO Community

SOCSCORE

(3) SOHR Human Rights

(Social Score)

(4) SODO Diversity \& Opportunity

(5) SOEQ Employment Quality

(6) SOHS Health \& Safety

(7) SOTD Training \& Development

\begin{tabular}{cl}
\hline Net Interest Margin (NIM) & $\begin{array}{l}\text { Ratio of net interest income to earning assets, which is } \\
\text { the sum of total investment earning interest or } \\
\text { dividends and net loans }\end{array}$ \\
\hline Tier1 Cap_Adq Ratio (TIER1) & Tier1 Capital Adequacy Ratio \\
\hline Non Performing Loans (NPL) & Ratio of non-performing loans to total loans \\
\hline Cost to Income Ratio (CIR) & Ratio of total interest expense to total interest income \\
\hline Excess Stock Return (ESR) & $\begin{array}{l}\text { Annual buy and hold return in excess of local market } \\
\text { return }\end{array}$ \\
\hline Size & The natural logarithm of book value of assets in USD \\
\hline LoanDeposit & Ratio of total loans to total deposits \\
\hline Pr.Crd.GDP & $\begin{array}{l}\text { Private credit by deposit money banks and other } \\
\text { financial institutions to GDP (\%) }\end{array}$ \\
\hline
\end{tabular}


Table A1. Cont.

\begin{tabular}{cl}
\hline \multicolumn{1}{c}{ Variables } & \multicolumn{1}{c}{ Definitions } \\
\hline & \multicolumn{1}{c}{ Average score of firm-level } \\
Mean_CSR SCORE (Country/Year) & $\begin{array}{l}\text { Governance/Environmental/Social (CSR) scores by } \\
\text { country and year }\end{array}$ \\
\hline \multirow{2}{*}{ Mean_CSR SCORE (Country/Industry) } & $\begin{array}{l}\text { Average score of firm-level } \\
\text { Governance/Environmental/Social (CSR) scores by } \\
\text { country and industry }\end{array}$ \\
\hline
\end{tabular}

CORPORATE GOVERNANCE SCORE (CGVSCORE): The corporate governance pillar measures a company's systems and processes, which ensure that its board members and executives act in the best interests of its long-term shareholders. It reflects a company's capacity, through its use of best management practices, to direct and control its rights and responsibilities through the creation of incentives, as well as checks and balances in order to generate long-term shareholder value.

Board of Directors/Board Functions (CGBF): The board of directors/board functions category measures a company's management commitment and effectiveness towards following best practice corporate governance principles related to board activities and functions. It reflects a company's capacity to have an effective board by setting up the essential board committees with allocated tasks and responsibilities.

Board of Directors/Board Structure (CGBS): The board of directors/board structure category measures a company's management commitment and effectiveness towards following best practice corporate governance principles related to a well-balanced membership of the board. It reflects a company's capacity to ensure a critical exchange of ideas and an independent decision-making process through an experienced, diverse and independent board.

Board of Directors/Compensation Policy (CGCP): The board of directors/compensation policy category measures a company's management commitment and effectiveness towards following best practice corporate governance principles related to competitive and proportionate management compensation. It reflects a company's capacity to attract and retain executives and board members with the necessary skills by linking their compensation to individual or company-wide financial or extra-financial targets.

Integration/Vision and Strategy (CGVS): The integration/vision and strategy category measures a company's management commitment and effectiveness towards the creation of an overarching vision and strategy integrating financial and extra-financial aspects. It reflects a company's capacity to convincingly show and communicate that it integrates the economic (financial), social and environmental dimensions into its day-to-day decision-making processes.

Shareholders/Shareholder Rights (CGSR): The shareholders/shareholder rights category measures a company's management commitment and effectiveness towards following best practice corporate governance principles related to a shareholder policy and equal treatment of shareholders. It reflects a company's capacity to be attractive to minority shareholders by ensuring them equal rights and privileges and by limiting the use of anti-takeover devices.

ENVIRONMENTAL SCORE (ENVSCORE): The environmental pillar measures a company's impact on living and non-living natural systems, including the air, land and water, as well as complete ecosystems. It reflects how well a company uses best management practices to avoid environmental risks and capitalize on environmental opportunities in order to generate long-term shareholder value.

Emission Reduction (ENER): The emission reduction category measures a company's management commitment and effectiveness towards reducing environmental emission in the production and operational processes. It reflects a company's capacity to reduce air emissions (greenhouse gases, F-gases, ozone-depleting substances, NOx and SOx, etc.), waste, hazardous waste, water discharges, spills or its impacts on biodiversity and to partner with environmental organizations to reduce the environmental impact of the company in the local or broader community. 
Product Innovation (ENPI): The product innovation category measures a company's management commitment and effectiveness towards supporting the research and development of eco-efficient products or services. It reflects a company's capacity to reduce the environmental costs and burdens for its customers, and thereby creating new market opportunities through new environmental technologies and processes or eco-designed, dematerialized products with extended durability.

Resource Reduction (ENRR): The resource reduction category measures a company's management commitment and effectiveness towards achieving an efficient use of natural resources in the production process. It reflects a company's capacity to reduce the use of materials, energy or water, and to find more eco-efficient solutions by improving supply chain management.

SOCIAL SCORE (SOCSCORE): The social pillar measures a company's capacity to generate trust and loyalty with its workforce, customers and society, through its use of best management practices. It is a reflection of the company's reputation and the health of its license to operate, which are key factors in determining its ability to generate long-term shareholder value.

Customer/Product Responsibility (SOPR): The customer/product responsibility category measures a company's management commitment and effectiveness towards creating value-added products and services upholding the customer's security. It reflects a company's capacity to maintain its license to operate by producing quality goods and services integrating the customer's health and safety, and preserving its integrity and privacy through accurate product information and labelling.

Society/Community (SOCO): The society/community category measures a company's management commitment and effectiveness towards maintaining the company's reputation within the general community (local, national and global). It reflects a company's capacity to maintain its license to operate by being a good citizen (donations of cash, goods or staff time, etc.), protecting public health (avoidance of industrial accidents, etc.) and respecting business ethics (avoiding bribery and corruption, etc.).

Society/Human Rights (SOHR): The society/human rights category measures a company's management commitment and effectiveness towards respecting the fundamental human rights conventions. It reflects a company's capacity to maintain its license to operate by guaranteeing the freedom of association and excluding child, forced or compulsory labor.

Workforce/Diversity and Opportunity (SODO): The workforce/diversity and opportunity category measures a company's management commitment and effectiveness towards maintaining diversity and equal opportunities in its workforce. It reflects a company's capacity to increase its workforce loyalty and productivity by promoting an effective life-work balance, a family friendly environment and equal opportunities regardless of gender, age, ethnicity, religion or sexual orientation.

Workforce/Employment Quality (SOEQ): The workforce/employment quality category measures a company's management commitment and effectiveness towards providing high-quality employment benefits and job conditions. It reflects a company's capacity to increase its workforce loyalty and productivity by distributing rewarding and fair employment benefits, and by focusing on long-term employment growth and stability by promoting from within, avoiding lay-offs and maintaining relations with trade unions.

Workforce/Health \& Safety (SOHS): The workforce/health \& safety category measures a company's management commitment and effectiveness towards providing a healthy and safe workplace. It reflects a company's capacity to increase its workforce loyalty and productivity by integrating into its day-to-day operations a concern for the physical and mental health, well-being and stress level of all employees.

Workforce/Training and Development (SOTD): The workforce/training and development category measures a company's management commitment and effectiveness towards providing training and development (education) for its workforce. It reflects a company's capacity to increase its intellectual capital, workforce loyalty and productivity by developing the workforce's skills, competences, employability and careers in an entrepreneurial environment. 


\section{Appendix B Sample countries and composition of the sample.}

Table A2. This table reports the number of firm/year observations available for banking sector in ASSET4 by country with availability of all variables for financial performance and responsibility scores. The sample period is from 2002 to 2015.

\begin{tabular}{|c|c|c|c|c|c|}
\hline Country & $\begin{array}{c}\text { \# of } \\
\text { Observations }\end{array}$ & Income Level & Legal Origin & $\begin{array}{l}\text { Supervisory } \\
\text { Authority }\end{array}$ & Supervision \\
\hline Australia & 67 & & Common & Single & NonCB \\
\hline Austria & 23 & & Civil & Multiple & NonCB \\
\hline Belgium & 23 & & Civil & Single & NonCB \\
\hline Brazil & 39 & Low & Civil & Single & CB \\
\hline Canada & 86 & & Common & Single & NonCB \\
\hline Chile & 23 & Low & Civil & Single & NonCB \\
\hline China & 67 & Low & Civil & Single & NonCB \\
\hline Colombia & 14 & Low & Civil & Single & NonCB \\
\hline Czech Republic & 8 & & Civil & Single & CB \\
\hline Denmark & 33 & High & Civil & Multiple & NonCB \\
\hline Egypt & 16 & & Civil & Single & $\mathrm{CB}$ \\
\hline Finland & 10 & & Civil & Single & NonCB \\
\hline France & 48 & & Civil & Single & CB \\
\hline Germany & 54 & & Civil & Multiple & NonCB \\
\hline Greece & 65 & Low & Civil & Single & CB \\
\hline Hong Kong & 61 & Low & Common & Single & $\mathrm{CB}$ \\
\hline Hungary & 5 & Low & Civil & Single & NonCB \\
\hline India & 57 & Low & Common & Single & CB \\
\hline Indonesia & 25 & Low & Civil & Single & $\mathrm{CB}$ \\
\hline Ireland & 25 & High & Common & Single & $\mathrm{CB}$ \\
\hline Israel & 26 & Low & Common & Single & $\mathrm{CB}$ \\
\hline Italy & 122 & Low & Civil & Single & $\mathrm{CB}$ \\
\hline Japan & 242 & & Civil & Single & NonCB \\
\hline Korea, Rep. & 41 & Low & Civil & Single & NonCB \\
\hline Malaysia & 53 & Low & Common & Single & CB \\
\hline Mexico & 18 & Low & Civil & Single & NonCB \\
\hline Netherlands & 4 & High & Civil & Single & CB \\
\hline Norway & 11 & High & Civil & Single & NonCB \\
\hline Philippines & 25 & Low & Civil & Single & CB \\
\hline Poland & 52 & Low & Civil & Single & NonCB \\
\hline Portugal & 23 & Low & Civil & Single & CB \\
\hline Qatar & 14 & & & Single & \\
\hline Russian Federation & 19 & Low & Civil & Single & $\mathrm{CB}$ \\
\hline Saudi Arabia & 10 & & Common & Single & $\mathrm{CB}$ \\
\hline Singapore & 34 & Low & Common & Single & $\mathrm{CB}$ \\
\hline South Africa & 34 & Low & Common & Single & $\mathrm{CB}$ \\
\hline Spain & 88 & Low & Civil & Single & CB \\
\hline Sweden & 47 & High & Civil & Single & NonCB \\
\hline Switzerland & 38 & High & Civil & Single & NonCB \\
\hline Taiwan, China & 60 & & Civil & Multiple & NonCB \\
\hline Thailand & 41 & Low & Common & Single & $\mathrm{CB}$ \\
\hline Turkey & 44 & Low & Civil & Single & $\mathrm{CB}$ \\
\hline United Arab Emir. & 5 & & & Single & $\mathrm{CB}$ \\
\hline United Kingdom & 87 & & Common & Single & NonCB \\
\hline United States & 545 & High & Common & Multiple & CB \\
\hline
\end{tabular}




\section{Appendix C The relationship between responsibility scores and financial performance.}

Table A3. This table reports OLS and WLS estimations for simultaneous equation system of the relationship between responsibility scores and bank specific financial performance measures for the effect of crisis years with CGVSCORE, ENVSCORE, and SOCSCORE. The definitions of variables are in Appendix A. All regressions control year fixed effects. The sample period is from 2002 to 2015 . Robust standard errors presented in brackets are clustered at the firm level, and *****, and * denote statistical significance at the $1 \%, 5 \%$, and $10 \%$ levels.

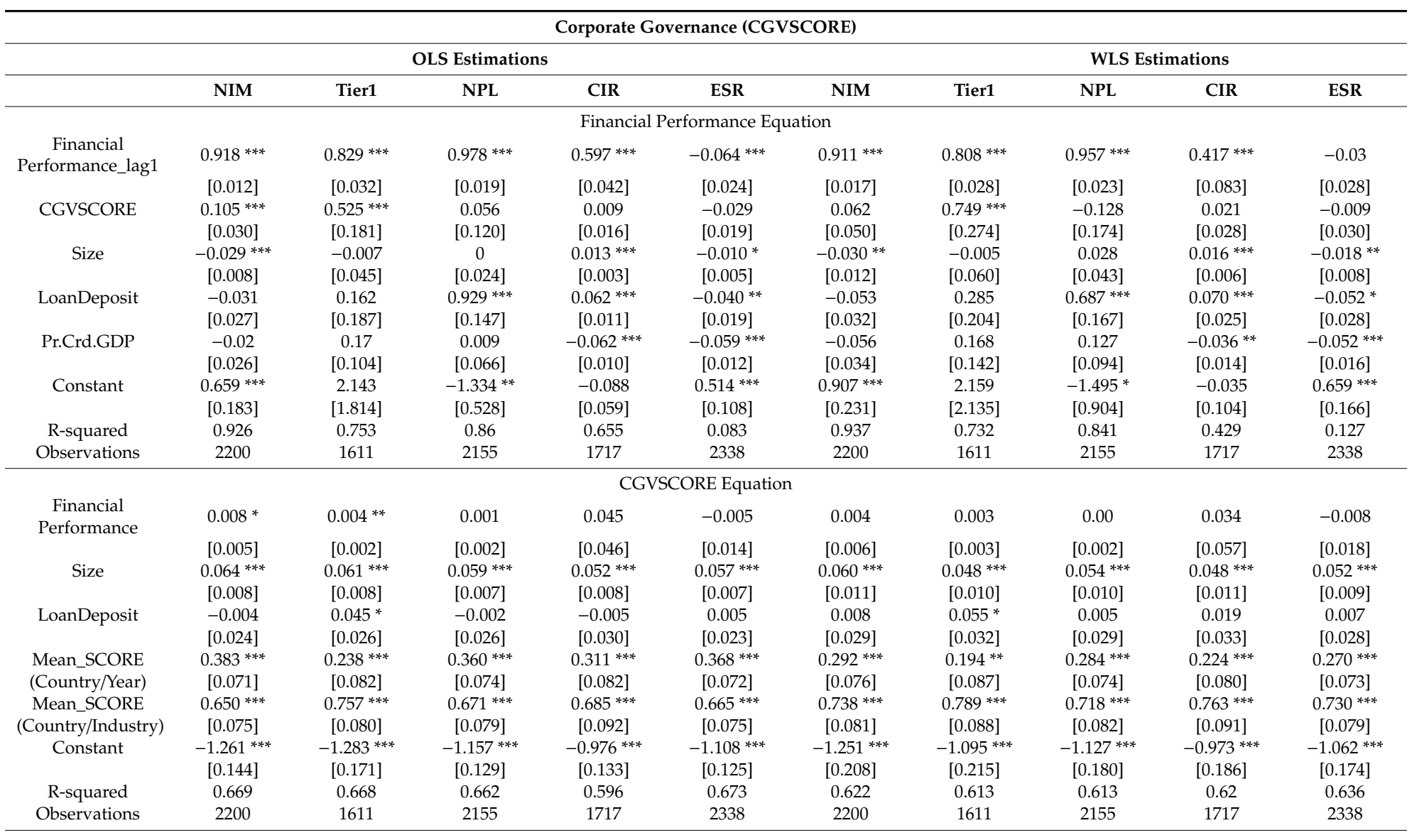


Table A3. Cont.

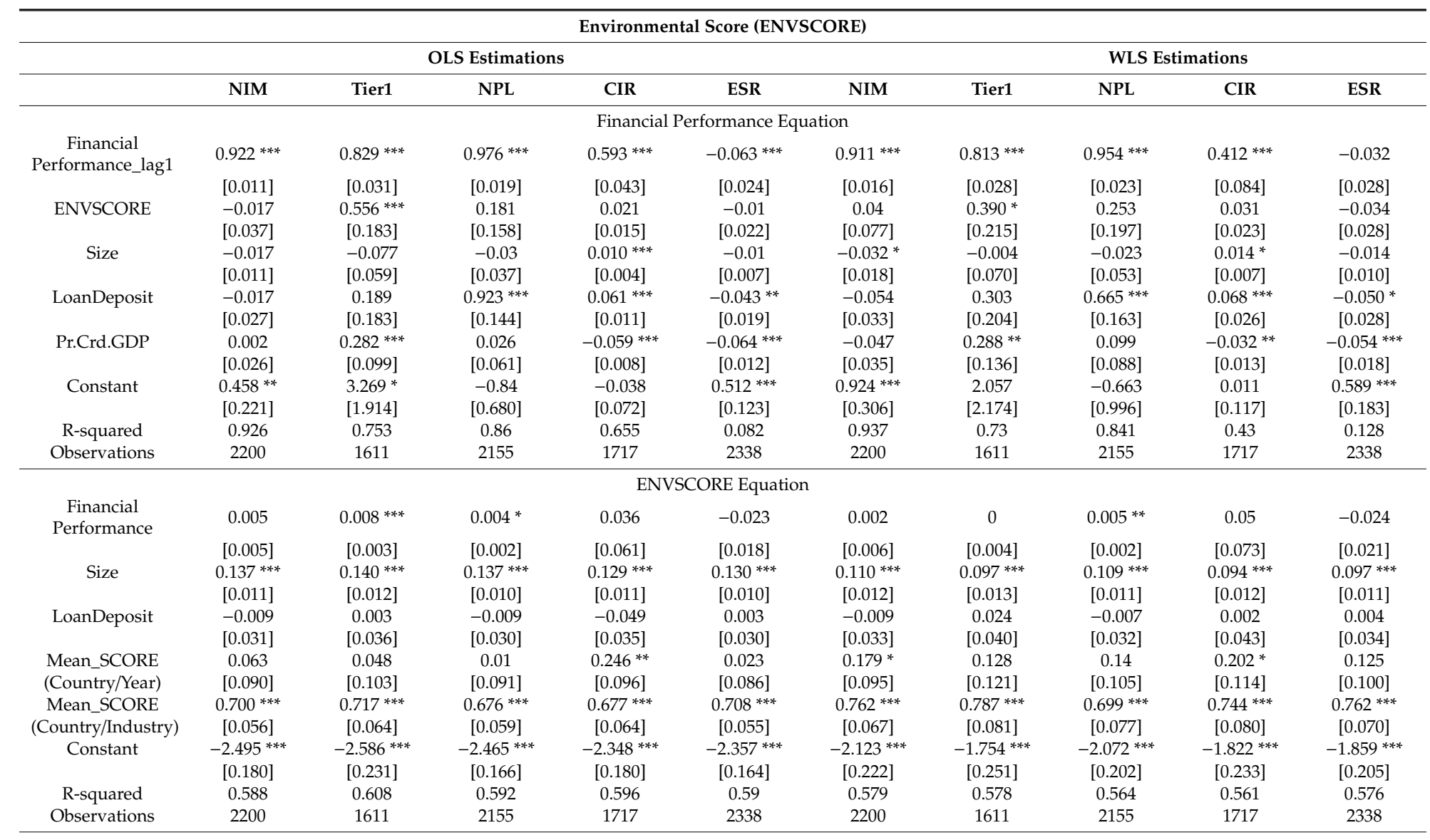


Table A3. Cont.

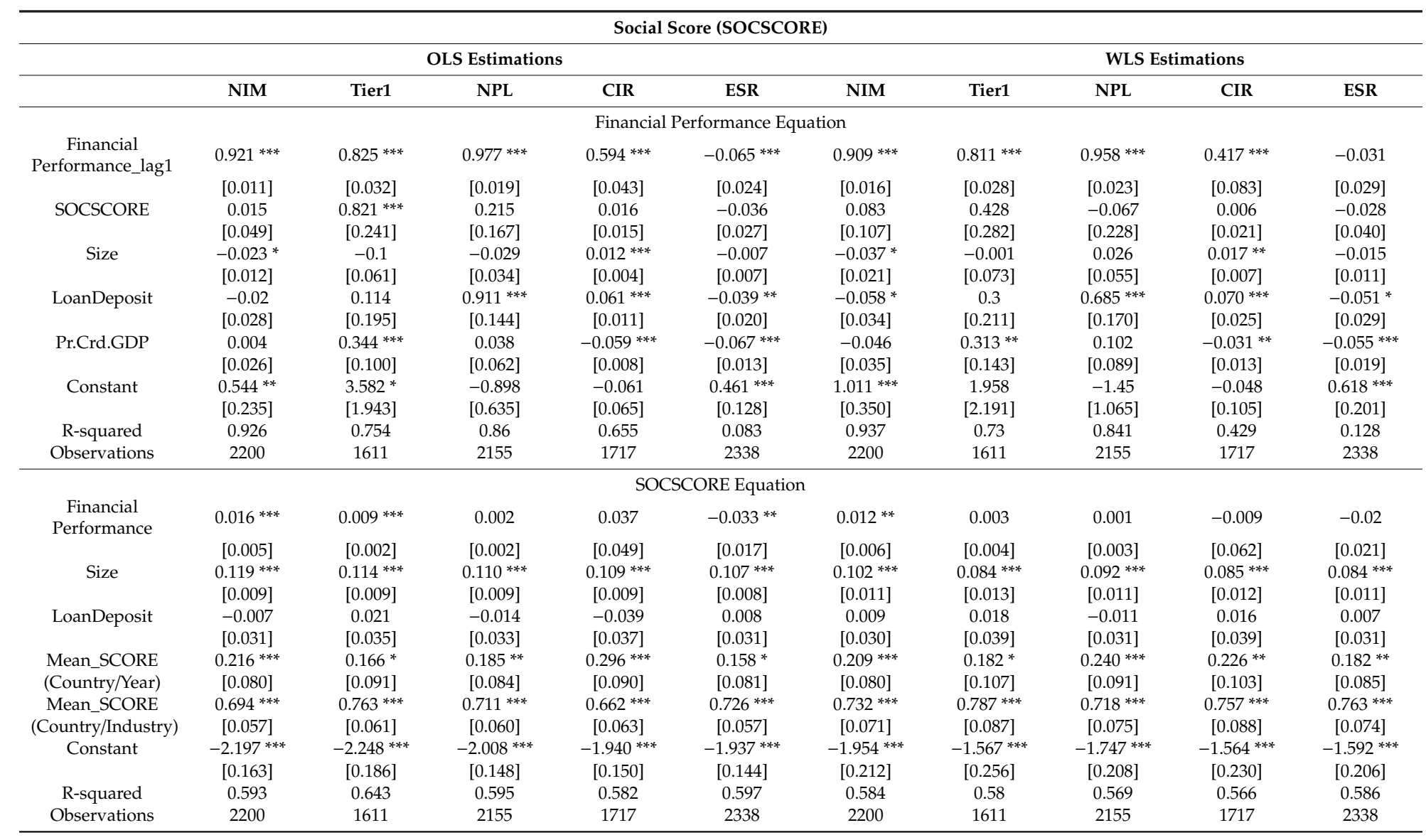


Appendix D VIF scores.

Table A4. This table reports Variance of Inflation Factor (VIF) scores in the main regressions.

\begin{tabular}{|c|c|c|c|c|c|}
\hline & NIM & Tier1 & NPL & CIR & ESR \\
\hline \multicolumn{6}{|c|}{ Financial Performance Equation } \\
\hline Financial Performance_lag1 & 1.29 & 1.28 & 1.15 & 1.65 & 1.09 \\
\hline CGVSCORE & 1.26 & 1.23 & 1.22 & 1.54 & 1.21 \\
\hline Size & 1.29 & 1.27 & 1.12 & 1.23 & 1.12 \\
\hline LoanDeposit & 1.19 & 1.16 & 1.2 & 1.19 & 1.18 \\
\hline Pr.Crd.GDP & 1.39 & 1.2 & 1.24 & 1.59 & 1.24 \\
\hline \multicolumn{6}{|c|}{ CGVSCORE Equation } \\
\hline Financial Performance & 1.15 & 1.2 & 1.21 & 1.69 & 1.09 \\
\hline Size & 1.2 & 1.16 & 1.08 & 1.14 & 1.08 \\
\hline LoanDeposit & 1.06 & 1.06 & 1.14 & 1.2 & 1.06 \\
\hline Mean_SCORE (Country/Year) & 4.57 & 5.02 & 4.6 & 4.2 & 4.77 \\
\hline Mean_SCORE (Country/Industry) & 4.77 & 4.97 & 4.87 & 4.28 & 4.94 \\
\hline \multicolumn{6}{|c|}{ Financial Performance Equation } \\
\hline Financial Performance_lag1 & 1.25 & 1.27 & 1.14 & 1.68 & 1.09 \\
\hline ENVSCORE & 1.84 & 1.91 & 1.9 & 1.85 & 1.85 \\
\hline Size & 1.93 & 2.06 & 1.88 & 1.83 & 1.83 \\
\hline LoanDeposit & 1.17 & 1.13 & 1.18 & 1.2 & 1.16 \\
\hline Pr.Crd.GDP & 1.22 & 1.11 & 1.14 & 1.2 & 1.14 \\
\hline \multicolumn{6}{|c|}{ ENVSCORE Equation } \\
\hline Financial Performance & 1.29 & 1.18 & 1.18 & 1.66 & 1.08 \\
\hline Size & 1.35 & 1.35 & 1.3 & 1.25 & 1.31 \\
\hline LoanDeposit & 1.26 & 1.23 & 1.28 & 1.51 & 1.23 \\
\hline Mean_SCORE (Country/Year) & 1.5 & 1.33 & 1.36 & 1.64 & 1.33 \\
\hline Mean_SCORE (Country/Industry) & 1.47 & 1.43 & 1.51 & 1.58 & 1.49 \\
\hline \multicolumn{6}{|c|}{ Financial Performance Equation } \\
\hline Financial Performance_lag1 & 1.26 & 1.29 & 1.13 & 1.67 & 1.09 \\
\hline SOCSCORE & 1.77 & 1.89 & 1.78 & 1.78 & 1.78 \\
\hline Size & 1.82 & 1.91 & 1.67 & 1.72 & 1.66 \\
\hline LoanDeposit & 1.2 & 1.17 & 1.2 & 1.2 & 1.19 \\
\hline Pr.Crd.GDP & 1.24 & 1.16 & 1.17 & 1.22 & 1.18 \\
\hline \multicolumn{6}{|c|}{ SOCSCORE Equation } \\
\hline Financial Performance & 1.17 & 1.19 & 1.18 & 1.66 & 1.08 \\
\hline Size & 1.31 & 1.25 & 1.19 & 1.22 & 1.2 \\
\hline LoanDeposit & 1.43 & 1.42 & 1.47 & 1.49 & 1.42 \\
\hline Mean_SCORE (Country/Year) & 1.63 & 1.6 & 1.66 & 1.62 & 1.61 \\
\hline Mean_SCORE (Country/Industry) & 1.61 & 1.53 & 1.61 & 1.63 & 1.6 \\
\hline
\end{tabular}


Appendix E Descriptive statistics of country sample along per capita income, legal origin, and organization of banking supervision.

Table A5. This table reports the number of firm/year observations and the mean values of responsibility scores and financial performance measures by per capita income, legal origin, and organization of banking supervision. The definitions of variables are in Appendix A. The sample period is from 2002 to 2015 . The significance of differences between means is based on a t-test for mean differences, and ${ }^{* * *}, * *$, and $*$ denote statistical significance at the $1 \%, 5 \%$, and $10 \%$ levels.

\begin{tabular}{|c|c|c|c|c|c|c|c|c|c|c|c|c|c|c|c|c|c|}
\hline \multicolumn{18}{|c|}{ Comparisons Based on Income Level with the Bottom and Top 40 Percentile of GDP per Capita by Year } \\
\hline & \multicolumn{8}{|c|}{ Low Income Countries } & \multicolumn{6}{|c|}{ High Income Countries } & \multirow{2}{*}{\multicolumn{3}{|c|}{$\begin{array}{c}\text { Low vs. High } \\
\text { Comparisons in Means }\end{array}$}} \\
\hline & \multicolumn{2}{|c|}{ Total } & \multicolumn{2}{|c|}{ Pre-Crisis } & \multicolumn{2}{|c|}{ Post-Crisis } & \multicolumn{2}{|c|}{ Comparison Total } & \multicolumn{3}{|c|}{ Pre-Crisis } & \multicolumn{2}{|c|}{ Post-Crisis } & \multirow{2}{*}{$\begin{array}{l}\text { Comparison } \\
\text { Post-Pre } \\
\end{array}$} & & & \\
\hline & $\mathbf{N}$ & Mean & $\mathbf{N}$ & Mean & $\mathbf{N}$ & Mean & Post - Pre & $\mathbf{N}$ & Mean & $\mathbf{N}$ & Mean & $\mathbf{N}$ & Mean & & Total & Pre-Crisis & Post-Crisis \\
\hline CGVSCORE & 1061 & 39.95 & 177 & 37.63 & 694 & 42.53 & $4.901^{* *}$ & 775 & 64.03 & 252 & 69.14 & 644 & 64.46 & $-4.68^{* * *}$ & $-24.07^{* * *}$ & $-31.51^{* * *}$ & $-21.93^{* * *}$ \\
\hline ENVSCORE & 1061 & 51.90 & 177 & .19 & 694 & 53.80 & & 775 & 41.75 & 252 & 40.10 & 644 & 41.39 & 1.29 & $10.14^{* * *}$ & $11.10^{* * *}$ & $12.41^{* * *}$ \\
\hline SOCSCORE & 1061 & 60.47 & 177 & .98 & 694 & 61.53 & 0.553 & 775 & 48.03 & 252 & 56.35 & 644 & 47.91 & $-8.44^{* * *}$ & $12.45^{* * *}$ & 4.63 & $13.62^{* * *}$ \\
\hline NIM & 1061 & & 177 & & 694 & & $0.008^{* * *}$ & & 029 & 252 & & 644 & 0.029 & $0.001 *$ & $0.002 * * *$ & $-0.003^{* * *}$ & $0.004^{* * *}$ \\
\hline Tier1 & 820 & 0.114 & 53 & 0.094 & 608 & & 0.025 & 61 & & 108 & & 493 & & 0.03 & $-0.011^{* * *}$ & 0.003 & $-0.009^{* * *}$ \\
\hline NPL & 971 & 0.041 & 158 & 0.018 & 634 & 0.048 & $0.031^{* * *}$ & 751 & 0.020 & 236 & 0.006 & 622 & 0.019 & $0.012 * * *$ & $0.021^{* * *}$ & $0.011^{* * *}$ & $0.030^{* * *}$ \\
\hline CIR & 796 & 0.469 & 116 & 0.551 & 563 & 0.446 & $-0.105^{* * *}$ & 744 & 0.351 & 231 & 0.500 & 620 & 0.340 & $-0.160 * * *$ & $0.118^{* * *}$ & $0.051^{* * *}$ & $0.105^{* * *}$ \\
\hline ESR & 1061 & 0.033 & 177 & 0.027 & 694 & 0.030 & 0.003 & 775 & -0.008 & 252 & -0.018 & 644 & 0.002 & $0.020 *$ & $0.041^{* * *}$ & $0.045^{* *}$ & 0.028 \\
\hline \multicolumn{18}{|c|}{ Comparisons Based on legal Origin } \\
\hline & \multicolumn{8}{|c|}{ Civil Law Countries } & \multicolumn{5}{|c|}{ Common Law Countries } & & \multicolumn{3}{|c|}{ Civil vs. Common } \\
\hline & \multicolumn{2}{|c|}{ Total } & \multicolumn{2}{|c|}{ Pre-Crisis } & \multicolumn{2}{|c|}{ Post-Crisis } & \multicolumn{2}{|c|}{ Comparison Total } & \multicolumn{3}{|c|}{ Pre-Crisis } & \multicolumn{2}{|c|}{ Post-Crisis } & Comparison & \multicolumn{3}{|c|}{ Comparisons in Means } \\
\hline & $\mathbf{N}$ & Mean & $\mathbf{N}$ & Mean & $\mathbf{N}$ & Mean & Post - Pre & $\mathbf{N}$ & Mean & $\mathbf{N}$ & Mean & $\mathbf{N}$ & Mean & Post-Pre & Total & Pre-Crisis & Post-Crisis \\
\hline CGVSCORE & 1184 & 33.80 & 298 & 30.69 & 683 & 35.81 & $5.118^{* * *}$ & 1116 & 63.11 & 307 & 69.91 & 636 & 60.24 & $-9.67^{* * *}$ & $-29.32 * * *$ & $-39.22 * * *$ & $-24.43^{* * *}$ \\
\hline ENVSCORE & 1184 & 53.27 & 298 & 50.52 & 683 & 55.23 & $4.709 * *$ & 1116 & 46.09 & 307 & 44.66 & 636 & 47.03 & 2.37 & $7.18^{* * *}$ & $5.86^{* *}$ & $8.20^{* * *}$ \\
\hline SOCSCORE & 1184 & 56.96 & 298 & 56.67 & 683 & 57.37 & 0.700 & 1116 & 54.15 & 307 & 59.55 & 636 & 51.39 & $-8.16^{* * *}$ & $2.81^{* *}$ & -2.88 & $5.98^{* * *}$ \\
\hline NIM & 1184 & 0.025 & 298 & 0.019 & 683 & 0.027 & $0.008^{* * *}$ & 1116 & 0.029 & 307 & 0.028 & 636 & 0.030 & $0.002^{* * *}$ & $-0.004^{* * *}$ & $-0.009^{* * *}$ & $-0.003^{* * *}$ \\
\hline Tier1 & 846 & 0.115 & 85 & 0.093 & 588 & 0.122 & $0.029^{* * *}$ & 907 & 0.120 & 126 & 0.092 & 616 & 0.128 & $0.036^{* * *}$ & $-0.005^{* * *}$ & 0.001 & $-0.006^{* * *}$ \\
\hline NPL & 1055 & 0.040 & 258 & 0.024 & 611 & 0.049 & $0.026^{* * *}$ & 1088 & 0.019 & 292 & 0.008 & 625 & 0.024 & $0.017^{* * *}$ & $0.021 * * *$ & $0.016^{* * *}$ & $0.025^{* * *}$ \\
\hline CIR & 757 & 0.477 & 132 & 0.618 & 513 & 0.426 & $-0.191 * * *$ & 1033 & 0.384 & 297 & 0.511 & 578 & 0.311 & $-0.200 * * *$ & $0.093^{* * *}$ & $0.106^{* * *}$ & $0.115^{* * *}$ \\
\hline ESR & 1184 & 0.026 & 298 & 0.048 & 683 & 0.013 & $-0.035^{* *}$ & 1116 & 0.015 & 307 & -0.021 & 636 & 0.035 & $0.056^{* * *}$ & 0.012 & $0.070^{* * *}$ & -0.022 \\
\hline
\end{tabular}


Table A5. Cont.

\begin{tabular}{|c|c|c|c|c|c|c|c|c|c|c|c|c|c|c|c|c|c|}
\hline \multicolumn{18}{|c|}{ Comparisons Based on Number of Supervisory Authorities } \\
\hline & \multicolumn{7}{|c|}{$\begin{array}{c}\text { Countries with Single Bank Supervisory } \\
\text { Authorities(Single) }\end{array}$} & \multicolumn{7}{|c|}{$\begin{array}{c}\text { Countries with Multiple Bank Supervisory } \\
\text { Authorities(Multiple) }\end{array}$} & \multirow{2}{*}{\multicolumn{3}{|c|}{$\begin{array}{l}\text { Single vs. Multiple } \\
\text { Comparisons in Means }\end{array}$}} \\
\hline & \multicolumn{2}{|c|}{ Total } & \multicolumn{2}{|c|}{ Pre-Crisis } & \multicolumn{2}{|c|}{ Post-Crisis } & \multirow{2}{*}{$\begin{array}{l}\text { Comparison } \\
\text { Post - Pre } \\
\end{array}$} & \multicolumn{2}{|c|}{ Total } & \multicolumn{2}{|c|}{ Pre-Crisis } & \multicolumn{2}{|c|}{ Post-Crisis } & \multirow{2}{*}{$\begin{array}{l}\text { Comparison } \\
\text { Post - Pre } \\
\end{array}$} & & & \\
\hline & $\mathbf{N}$ & Mean & $\mathbf{N}$ & Mean & $\mathbf{N}$ & Mean & & $\mathbf{N}$ & Mean & $\mathbf{N}$ & Mean & $\mathbf{N}$ & Mean & & Total & Pre-Crisis & Post-Crisis \\
\hline CGVSCORE & 1717 & 41.32 & 395 & 42.56 & 1040 & 41.61 & -0.949 & 715 & 57.37 & 210 & 65.70 & 396 & 52.74 & $-12.97^{* * *}$ & $-16.05^{* * *}$ & $-23.14 * * *$ & $-11.13 * * *$ \\
\hline ENVSCORE & 1717 & 54.24 & 395 & 55.93 & 1040 & 54.36 & -1.571 & 715 & 35.90 & 210 & 31.77 & 396 & 38.10 & $6.33 * *$ & $18.34^{* * *}$ & $24.17^{* * *}$ & $16.26^{* * *}$ \\
\hline SOCSCORE & 1717 & 59.26 & 395 & 62.06 & 1040 & 58.36 & $-3.702 * *$ & 715 & 43.13 & 210 & 50.75 & 396 & 38.72 & $-12.03 * * *$ & $16.13^{* * *}$ & $11.32 * * *$ & $19.64^{* * *}$ \\
\hline NIM & 1717 & 0.026 & 395 & 0.020 & 1040 & 0.028 & $0.008^{* * *}$ & 715 & 0.030 & 210 & 0.030 & 396 & 0.030 & 0.000 & $-0.004^{* * *}$ & $-0.010^{* * *}$ & $-0.002 * *$ \\
\hline Tier1 & 1289 & 0.116 & 115 & 0.094 & 928 & 0.122 & $0.028^{* * *}$ & 563 & 0.122 & 96 & 0.090 & 364 & 0.131 & $0.041^{* * *}$ & $-0.006^{* * *}$ & 0.004 & $-0.009 * * *$ \\
\hline NPL & 1585 & 0.035 & 352 & 0.019 & 966 & 0.041 & $0.022 * * *$ & 681 & 0.017 & 198 & 0.008 & 380 & 0.020 & $0.013^{* * *}$ & $0.017^{* * *}$ & $0.012 * * *$ & $0.021^{* * *}$ \\
\hline CIR & 1219 & 0.474 & 231 & 0.596 & 810 & 0.430 & $-0.166^{* * *}$ & 694 & 0.326 & 198 & 0.484 & 392 & 0.234 & $-0.250^{* * *}$ & $0.148^{* * *}$ & $0.112 * * *$ & $0.196^{* * *}$ \\
\hline ESR & 1717 & 0.036 & 395 & 0.026 & 1040 & 0.030 & $0.004^{* *}$ & 715 & -0.006 & 210 & -0.011 & 396 & 0.015 & 0.026 & $0.042^{* * *}$ & $0.036^{*}$ & 0.015 \\
\hline \multicolumn{18}{|c|}{ Comparisons Based on Central Bank Supervision } \\
\hline & \multicolumn{7}{|c|}{$\begin{array}{l}\text { Countries with Supervision Outside of the Central } \\
\text { Bank(Non CB) }\end{array}$} & \multicolumn{7}{|c|}{ Countries with Supervision by the Central Bank(CB) } & \multicolumn{3}{|c|}{ Non CB vs. CB } \\
\hline & \multicolumn{2}{|c|}{ Total } & \multicolumn{2}{|c|}{ Pre-Crisis } & \multicolumn{2}{|c|}{ Post-Crisis } & Comparison & \multicolumn{2}{|c|}{ Total } & \multicolumn{2}{|c|}{ Pre-Crisis } & \multicolumn{2}{|c|}{ Post-Crisis } & Comparison & \multicolumn{3}{|c|}{ Comparisons in Means } \\
\hline & $\mathbf{N}$ & Mean & $\mathbf{N}$ & Mean & $\mathbf{N}$ & Mean & Post - Pre & $\mathbf{N}$ & Mean & $\mathbf{N}$ & Mean & $\mathbf{N}$ & Mean & Post - Pre & Total & Pre-Crisis & Post-Crisis \\
\hline CGVSCORE & 1001 & 38.24 & 248 & 43.72 & 596 & 36.11 & $-7.616^{* * *}$ & 1417 & 51.90 & 357 & 55.36 & 828 & 51.33 & $-4.04 * *$ & $-13.65^{* * *}$ & $-11.64^{* * *}$ & $-15.22 * * *$ \\
\hline ENVSCORE & 1001 & 55.46 & 248 & 56.64 & 596 & 55.19 & $-1.452^{* * *}$ & 1417 & 44.51 & 357 & 41.22 & 828 & 46.54 & $5.32 * *$ & $10.95^{* * *}$ & $15.42 * * *$ & $8.65^{* * *}$ \\
\hline SOCSCORE & 1001 & 56.02 & 248 & 61.34 & 596 & 53.66 & -7.680 & 1417 & 53.76 & 357 & 55.91 & 828 & 52.88 & -3.03 & $2.26^{*}$ & $5.43 * *$ & 0.77 \\
\hline NIM & 1001 & 0.020 & 248 & 0.017 & 596 & 0.022 & $0.005^{* * *}$ & 1417 & 0.032 & 357 & 0.028 & 828 & 0.033 & $0.005^{* * *}$ & $-0.011^{* * *}$ & $-0.011 * * *$ & $-0.011^{* * *}$ \\
\hline Tier1 & 705 & 0.113 & 75 & 0.092 & 497 & 0.119 & $0.027^{* * *}$ & 1133 & 0.120 & 136 & 0.093 & 783 & 0.128 & $0.035^{* * *}$ & $-0.008^{* * *}$ & -0.001 & $-0.009 * * *$ \\
\hline NPL & 900 & 0.023 & 223 & 0.019 & 534 & 0.025 & $0.006^{* * *}$ & 1352 & 0.034 & 327 & 0.013 & 800 & 0.043 & $0.030 * * *$ & $-0.011^{* * *}$ & $0.006^{* * *}$ & $-0.018^{* * *}$ \\
\hline CIR & 742 & 0.484 & 157 & 0.656 & 489 & 0.416 & $-0.239 * * *$ & 1157 & 0.380 & 272 & 0.479 & 701 & 0.331 & $-0.148^{* * *}$ & $0.104^{* * *}$ & $0.176^{* * *}$ & $0.085^{* * *}$ \\
\hline ESR & 1001 & 0.039 & 248 & 0.026 & 596 & 0.041 & 0.015 & 1417 & 0.012 & 357 & 0.004 & 828 & 0.014 & 0.010 & $0.027^{* *}$ & 0.022 & $0.027^{* *}$ \\
\hline
\end{tabular}




\section{References}

1. Cull, R.; Demirgüç-Kunt, A.; Lin, J.Y. Financial structure and economic development: A deassessment. World Bank Econ. Rev. 2013, 27, 470-475. [CrossRef]

2. Heal, G. When Principles Pay; Columbia Business School Publishing: New York, NY, USA; Chichester, UK, 2008.

3. Amnesty International. Banks, Arms and Human Rights Violations; Amnesty International: Luxembourg, 2016.

4. Anginer, D.; Demirguc-Kunt, A.; Huizinga, H.; Ma, K. Corporate governance and bank capitalization strategies. J. Financ. Intermediat. 2016, 26, 1-27. [CrossRef]

5. Hu, V.; Scholtens, B. Corporate social responsibility policies of commercial banks in developing countries. Sustain. Dev. 2014, 22, 276-288. [CrossRef]

6. Wu, M.W.; Shen, C.H. Corporate social responsibility in the banking industry: Motives and financial performance. J. Bank. Financ. 2013, 37, 3529-3547. [CrossRef]

7. Jo, H.; Kim, H.; Park, K. Corporate environmental responsibility and firm performance in the financial services sector. J. Bus. Ethics 2015, 131, 257-284. [CrossRef]

8. Chih, H.L.; Chih, H.H.; Chen, T.Y. On the determinants of corporate social responsibility: International evidence on the financial industry. J. Bus. Ethics 2010, 93, 115-135. [CrossRef]

9. Banking on Climate Change. Fossil Fuel Finance Report Card 2017; BankTrack, Rainforest Action Network, Sierra Club, Oilchange International: Washington, DC, USA, 2017.

10. Acharya, V.V.; Afonso, G.; Kovner, A. How do global banks scramble for liquidity? Evidence from the asset-backed commercial paper freeze of 2007. J. Financ. Intermediat. 2017, 30, 1-34. [CrossRef]

11. Altunbas, Y.; Manganelli, S.; Marques-Ibanez, D. Realized bank risk during the great recession. J. Financ. Intermediat. 2017, 32, 29-44. [CrossRef]

12. Calomiris, C.W.; Flandreau, M.; Laeven, L. Political foundations of the lender of last resort: A global historical narrative. J. Financ. Intermediat. 2016, 28, 48-65. [CrossRef]

13. Claessens, S. Global banking: Recent developments and insights from research. Rev. Financ. 2017, 21, 1513-1555. [CrossRef]

14. Basle Committee on Banking Supervision. Corporate Governance Principles for Banks; Bank for International Settlements: Basel, Switzerland, 2015.

15. Cornett, M.M.; Erhemjamts, O.; Tehranian, H. Greed or good deeds: An examination of the relation between corporate social responsibility and the financial performance of U.S. commercial banks around the financial crisis. J. Bank. Financ. 2016, 30, 137-159. [CrossRef]

16. Badía, G.; Pina, V.; Torres, L. Financial performance of government bond portfolios based on environmental, social and governance criteria. Sustainability 2019, 11, 2514. [CrossRef]

17. Shah, A.A.; Wu, D.; Korotkov, V. Are sustainable banks efficient and productive? A data envelopment analysis and the Malmquist productivity index analysis. Sustainability 2019, 11, 2398. [CrossRef]

18. Neanidis, K.C. Volatile capital flows and economic growth: The role of banking supervision. J. Financ. Stab. 2019, 40, 77-93. [CrossRef]

19. Dixit, A.K. How business community institutions can help fight corruption. World Bank Econ. Rev. 2015, 29, S25-S47. [CrossRef]

20. Kotchen, M.J.; Negi, N.K. Cofinancing in environment and development: Evidence from the Global Environment Facility. World Bank Econ. Rev. 2019, 33, 41-62. [CrossRef]

21. Cull, R.; Demirgüç-Kunt, A.; Murdoch, J. The microfinance business model: Enduring subsidy and modest profit. World Bank Econ. Rev. 2018, 32, 221-244. [CrossRef]

22. Huang, Y. Is economic volatility detrimental to global sustainability? World Bank Econ. Rev. 2011, 26, 128-146. [CrossRef]

23. Liang, H.; Renneboog, L. On the foundations of corporate social responsibility. J. Financ. 2017, 72, 853-909. [CrossRef]

24. Lins, K.V.; Servaes, H.; Tamayo, A. Social capital, trust, and firm performance: The value of corporate social responsibility during the financial crisis. J. Financ. 2017, 72, 1785-1823. [CrossRef]

25. Goss, A.; Roberts, G.S. The impact of corporate social responsibility on the cost of bank loans. J. Bank. Financ. 2011, 35, 1794-1810. [CrossRef] 
26. Wu, M.W.; Shen, C.H.; Chen, T.H. Application of multi-level matching between financial performance and corporate social responsibility in the banking industry. Rev. Quant. Financ. Account. 2017, 49, $29-63$. [CrossRef]

27. Goldsmith, R.W. Financial Structure and Development; Yale University Press: New Haven, CT, USA; London, UK, 1969.

28. Fama, E.F. What's different about banks? J. Monet. Econ. 1985, 15, 29-39. [CrossRef]

29. Boyd, J.H.; Prescott, E.C. Financial intermediary-coalitions. J. Econ. Theory 1986, 38, 211-232. [CrossRef]

30. Cheng, B.; Ioannou, I.; Serafeim, G. Corporate social responsibility and access to finance. Strateg. Manag. J. 2014, 35, 1-23. [CrossRef]

31. Chatterji, A.K.; Levine, D.I.; Toffel, M.W. How well do social ratings actually measure corporate social responsibility? J. Econ. Manag. Strategy 2009, 18, 125-169. [CrossRef]

32. Rice, T.; Rose, J. When good investments go bad: The contraction in community bank lending after the GSE 2008 takeover. J. Financ. Intermediat. 2016, 26, 68-88. [CrossRef]

33. Bouslah, K.; Kryzanowski, L.; M'Zali, B. Social performance and firm risk: Impact of the financial crisis. J. Bus. Ethics 2018, 149, 643-669. [CrossRef]

34. Gjølberg, M. The origin of corporate social responsibility: Global forces or national legacies? Socio-Econ. Rev. 2009, 7, 605-637. [CrossRef]

35. Barth, J.R.; Caprio, G., Jr.; Levine, R. Bank regulation and supervision in 180 countries from 1999 to 2011. J. Financ. Econ. Policy 2013, 5, 111-219. [CrossRef]

36. Dahlsrud, A. How corporate social responsibility is defined: An analysis of 37 definitions. Corp. Soc. Responsib. Environ. Manag. 2008, 15, 1-13. [CrossRef]

37. Commission of the European Communities. Promoting a European Framework for Corporate Social Responsibilities; COM (2001) 366 Final; Commission of the European Communities: Brussels, Belgium, 2001.

38. Margolis, J.D.; Walsh, J.P. People and Profits? The Search for a Link between a Company's Social and Financial Performance; Lawrence Erlbaum: Mahwah, NJ, USA, 2001.

39. Bénabou, R.; Tirole, J. Incentives and prosocial behavior. Am. Econ. Rev. 2006, 96, 1652-1678. [CrossRef]

40. Bénabou, R.; Tirole, J. Individual and corporate social responsibility. Economica 2010, 77, 1-19. [CrossRef]

41. Friede, G.; Busch, T.; Bassen, A. ESG and financial performance: Aggregated evidence from more than 2000 empirical studies. J. Sustain. Financ. Invest. 2015, 5, 210-233. [CrossRef]

42. Margolis, J.D.; Elfenbein, H.A.; Walsh, J.P. Does It Pay to Be Good ... and Does It Matter? A Meta-Analysis of the Relationship between Corporate Social and Financial Performance. 1 Marth 2009. Available online: http://ssrn.com/abstract=1866371 (accessed on 30 April 2019).

43. Waddock, S.; Graves, S. Finding the link between stakeholder relations and quality of management. J. Invest. 1997, 6, 20-24. [CrossRef]

44. Cottrill, H. Corporate Social Responsibility and the marketplace. J. Bus. Ethics 1990, 9, 723-729. [CrossRef]

45. Boutin-Dufresne, F.; Savaria, P. Corporate Social Responsibility and financial risk. J. Invest. 2004, 13, 57-66. [CrossRef]

46. Simpson, G.; Kohers, T. The link between corporate social and financial performance: Evidence from the banking industry. J. Bus. Ethics 2002, 35, 97-109. [CrossRef]

47. Fernando, C.S.; Sharfman, M.P.; Uysal, V.B. Corporate environmental policy and shareholder value: Following the smart money. J. Financ. Quant. Anal. 2017, 52, 2013-2051. [CrossRef]

48. Ferrell, A.; Liang, H.; Renneboog, L. Socially responsible firms. J. Financ. Econ. 2016, 122, 585-605. [CrossRef]

49. Preston, L.E.; O'Bannon, D.P. The corporate social-financial performance relationship: A typology and analysis. Bus. Soc. 1997, 36, 419-429. [CrossRef]

50. Rivera, J.M.; Muňoz, M.J.; Moneva, J.M. Revisiting the relationship between corporate stakeholder commitment and social and financial performance. Sustain. Dev. 2017, 25, 482-494. [CrossRef]

51. Freeman, R.E. Strategic Management: A Stakeholder Approach; Pitman: Boston, MA, USA, 1984.

52. Baird, P.L.; Geylani, P.C.; Roberts, J.A. Corporate social and financial performance re-examined: Industry effects in a linear mixed model analysis. J. Bus. Ethics 2012, 109, 367-388. [CrossRef]

53. Dimson, E.; Karakaş, O.; Li, X. Active ownership. Rev. Financ. Stud. 2015, 28, 3225-3268. [CrossRef]

54. Friedman, M. The social responsibility of business is to increase its profits. New York Times Magazine, 13 September 1970; pp. 32-33. 
55. Harris, M.; Raviv, A. Control of corporate decisions: Shareholders vs. management. Rev. Financ. Stud. 2010, 23, 4115-4147. [CrossRef]

56. McGuire, J.B.; Sundgren, A.; Schneeweis, T. Corporate social responsibility and firm performance. Acad. Manag. J. 1988, 31, 854-872.

57. Draper, D.W.; Hoag, J.W. Financial intermediation and the theory of agency. J. Financ. Quant. Anal. 1978, 13, 595-611. [CrossRef]

58. Jensen, M.C. Agency costs of free cash flow, corporate finance, and takeovers. Am. Econ. Rev. 1986, 76, 323-329.

59. Whelan, T.; Fink, C. The comprehensive business case for sustainability. Harv. Bus. Rev. 2016, $21,2012$.

60. Borisov, A.; Goldman, E.; Gupta, N. The corporate value of (corrupt) lobbying. Rev. Financ. Stud. 2016, 29, 1039-1071. [CrossRef]

61. Zeume, S. Bribes and firm value. Rev. Financ. Stud. 2017, 30, 1457-1489. [CrossRef]

62. Tobin, J. Financial intermediaries. In The New Palgrave, Money; Eatwell, J., Milgate, M., Newman, P., Eds.; MacMillan: London/Basingstoke, UK, 1987; pp. 157-174.

63. Pyle, D.H. On the theory of financial intermediation. J. Financ. 1971, 26, 737-747. [CrossRef]

64. Pyle, D.H. Descriptive theories of financial institutions under uncertainty. J. Financ. Quant. Anal. 1972, 14, 267-291. [CrossRef]

65. Bolton, B. Corporate Social Responsibility and Bank Performance. 2013. Available online: https://papers. ssrn.com/sol3/papers.cfm?abstract_id=2277912 (accessed on 4 October 2018).

66. Scholtens, B. Financial and social performance of socially responsible investments in The Netherlands. Corp. Gov. Int. Rev. 2007, 15, 1090-1105. [CrossRef]

67. Menassa, E. Corporate social responsibility. An exploratory study of the quality and extent of social disclosures by Lebanese commercial banks. J. Appl. Account. Res. 2010, 11, 4-23. [CrossRef]

68. Callado-Munoz, F.J.; Utrero-Gonzalez, N. Does it pay to be socially responsible? Evidence from Spain's retail banking sector. Eur. Financ. Manag. 2011, 17, 755-787. [CrossRef]

69. Uddin, S.; Islam, M.Z.; Hasan, I. Corporate social responsibility and financial performance linkage-Evidence from the banking sector of Bangladesh. J. Organ. Manag. 2012, 1, 14-21.

70. Saxena, M.; Kohli, A.S. Impact of corporate social responsibility on corporate sustainability: A study of the Indian banking industry. IUP J. Corp. Gov. 2012, 11, 1-16.

71. Kamal, M. The Role of Corporate Social Responsibility (CSR) in the Egyptian Banking Sector. MPRA_working paper_44697 2013. Available online: https://papers.ssrn.com/sol3/papers.cfm?abstract_id=2227621 or http://dx.doi.org/10.2139/ssrn.2227621 (accessed on 15 June 2019). [CrossRef]

72. Adewale, M.T.; Rahmon, T.A. Does corporate social responsibility improve an organization's financial performance? Evidence from Nigerian banking sector. IUP J. Corp. Gov. 2014, 13, 52-60.

73. Malik, M.S.; Nadeem, M. Impact of corporate social responsibility on the financial performance of banks in Pakistan. Int. Lett. Soc. Humanist. Sci. 2014, 21, 9-19.

74. Paulik, J.; Majková, M.S.; Tykva, T.; Cervinka, M. Application of the CSR measuring model in commercial banking in relation to their financial performance. Econ. Sociol. 2015, 8, 64-81. [CrossRef] [PubMed]

75. Soana, M.G. The relationship between corporate social performance and corporate financial performance in the banking sector. J. Bus. Ethics 2011, 104, 133-148. [CrossRef]

76. Ciciretti, R.; Kobeissi, N.; Zhu, Y. Corporate social responsibility and financial performance: An analysis of bank community responsibility. Int. J. Bank. Account. Financ. 2014, 5, 342-373. [CrossRef]

77. Mallin, C.; Hisham, F.; Kean, O.W. Corporate social responsibility and financial performance in Islamic banks. J. Econ. Behav. Organ. 2014, 103, S21-S38. [CrossRef]

78. Shen, C.H.; Wu, M.W.; Chen, T.H.; Fange, H. To engage or not to engage in corporate social responsibility: Empirical evidence from global banking sector. Econ. Model. 2016, 55, 207-225. [CrossRef]

79. Ioannou, I.; Serafeim, G. The impact of corporate social responsibility on investment recommendations: Analysts' perceptions and shifting institutional logics. Strateg. Manag. J. 2015, 36, 1053-1081. [CrossRef]

80. Platonova, E.; Asutay, M.; Dixon, R.; Mohammad, S. The impact of corporate social responsibility disclosure on financial performance: Evidence from the GCC Islamic banking sector. J. Bus. Ethics 2018, 1, 69-78. [CrossRef]

81. Orlitzky, M.; Schmidt, F.L.; Rynes, S.L. Corporate social and financial performance: A meta-analysis. Organ. Stud. 2003, 24, 403-441. [CrossRef] 
82. Dam, L.; Scholtens, B. Toward a theory of responsible investing: On the economic foundations of corporate social responsibility. Resour. Energy Econ. 2015, 41, 103-121. [CrossRef]

83. Kindleberger, C.P. A Financial History of Western Europe, 2nd ed.; Oxford University Press: New York, NY, USA; Oxford, UK, 1993.

84. Kane, E.J. Good intentions and unintended evil: The case against selective credit allocation. J. Money Credit Bank. 1977, 9, 55-69. [CrossRef]

85. Kane, E.J. What kind of multinational arrangements might best enhance world welfare? Pac. Basin Financ. J. 2003, 11, 413-428. [CrossRef]

86. Ayadi, R.; Llewellyn, D.T.; Schmidt, R.H.; Arbak, E.; De Groen, W.P. Investigating Diversity in the Banking Sector in Europe; Centre for European Policy Studies: Brussels, Belgium, 2010.

87. Hagendorff, J. Corporate governance in banking. In The Oxford Handbook of Banking, 2nd ed.; Berger, A.N., Molyneux, P., Wilson, J.O.S., Eds.; Oxford University Press: Oxford, UK, 2015; pp. 139-159.

88. El Ghoul, S.; Guedhami, O.; Hakkon, K.; Park, K. Corporate environmental responsibility and the cost of capital: International evidence. J. Bus. Ethics 2018, 149, 335-361. [CrossRef]

89. Gonenc, H.; Scholtens, B. Environmental and financial performance of fossil fuel firms: A closer inspection of their interaction. Ecol. Econ. 2017, 132, 307-328. [CrossRef]

90. Halbritter, G.; Dorfleitner, G. The wages of social responsibility-Where are they? A critical review of ESG investing. Rev. Financ. Econ. 2015, 26, 25-35. [CrossRef]

91. Stellner, C.; Klein, C.; Zwergel, B. Corporate social responsibility and Eurozone corporate bonds: The moderating role of country sustainability. J. Bank. Financ. 2015, 59, 538-549. [CrossRef]

92. Semenova, N.; Hassel, L.G. On the validity of environmental performance metrics. J. Bus. Ethics 2015, 132, 249-258. [CrossRef]

93. Escrig-Olmeo, E.; Muňoz-Torres, M.J.; Fernández-Izquierdo, M.A.; Rivera-Lirio, J.M. Measuring corporate environmental performance: A methodology for sustainable development. Bus. Strategy Environ. 2017, 26, 142-162. [CrossRef]

94. Wu, M.L. Corporate social performance and corporate financial performance and firm size: A meta-analysis. J. Am. Acad. Bus. 2006, 8, 163-171.

95. Scholtens, B. A note on the interaction between corporate social responsibility and financial performance. Ecol. Econ. 2008, 68, 46-55. [CrossRef]

96. La Porta, R.; López-de-Silanes, F.; Shleifer, A. The economic consequence of legal origins. J. Econ. Lit. 2008, 46, 285-332. [CrossRef]

97. Babecký, J.; Havránek, T.; Matějů, J.; Rusnák, M.; Šmídková, K.; Vašíček, B. Banking, Debt, and Currency Crises. Early Warning Indicators for Developed Countries. ECB Working Paper 1485, 2012. Available online: https://ssrn.com/abstract=2162901 (accessed on 15 June 2018).

98. Sapienza, P.; Zingales, L. A trust crisis. Int. Rev. Financ. 2012, 12, 123-131. [CrossRef]

99. Lins, K.V.; Volpin, P.; Wagner, H. Does family control matter? International evidence from the 2008-2009 financial crisis. Rev. Financ. Stud. 2013, 26, 2583-2619. [CrossRef]

100. Gormley, T.A.; Matsa, D.A. Common errors: How to (and not to) control for unobserved heterogeneity. Rev. Financ. Stud. 2013, 27, 617-661. [CrossRef]

101. Erickson, T.; Jiang, C.H.; Whited, T.M. Minimum distance estimation of the errors-in-variables model using linear cumulant equations. J. Econ. 2014, 183, 211-221. [CrossRef]

(C) 2019 by the authors. Licensee MDPI, Basel, Switzerland. This article is an open access article distributed under the terms and conditions of the Creative Commons Attribution (CC BY) license (http://creativecommons.org/licenses/by/4.0/). 